\title{
Miniature fiber-optic multicavity Fabry-Perot interferometric biosensor
}

\author{
Yan Zhang \\ Dissertation submitted to the faculty of the \\ Virginia Polytechnic Institute and State University \\ in partial fulfillment of the requirements for the degree of \\ Doctor of Philosophy \\ In \\ Electrical Engineering
}

Anbo Wang, Chair

James R. Heflin

Ira Jacobs

Gary R. Pickrell

Ahmad Safaai-Jazi

December 6, 2005

Blacksburg, Virginia

Keywords: Fabry-Perot interferometry, Fiber optic biosensor, Polyelectrolyte selfassembly, Temperature compensation

CCopyright 2005, Yan Zhang 


\title{
Miniature fiber-optic multicavity Fabry-Perot interferometric biosensor
}

\author{
Yan Zhang
}

\begin{abstract}
(ABSTRACT)
Fiber-optic Fabry-Perot interferometric (FFPI) sensors have been widely used due to their high sensitivity, ease of fabrication, miniature size, and capability for multiplexing. However, direct measurement of self-assembled thin films, receptor immobilization process or biological reaction is limited in the FFPI technique due to the difficulty of forming Fabry-Perot cavities by the thin film itself. Novel methods are needed to provide an accurate and reliable measurement for monitoring the thin-film growth in the nanometer range and under various conditions.

In this work, two types of fiber-optic multicavity Fabry-Perot interferometric (MFPI) sensors with built-in temperature compensation were designed and fabricated for thin-film measurement, with applications in chemical and biological sensing. Both the tubing-based MFPI sensor and microgap MFPI sensor provide simple, yet high performance solutions for thin-film sensing. The temperature dependence of the sensing cavity is compensated by extracting the temperature information from a second multiplexed cavity. This provides the opportunity to examine the thin-film characteristics under different environment temperatures.

To demonstrate the potential of this structure for practical applications, immunosensors were fabricated and tested using these structures. Self-assembled polyelectrolytes served as a precursor film for immobilization of antibodies to ensure they retain their biological activity. This not only provides a convenient method for protein immobilization but also presents the possibility of increasing the binding capacity and sensitivity by incorporating multilayers of antibodies into polyelectrolyte layers. The steady-state measurement demonstrated the surface concentration and binding ratio of the
\end{abstract}


immunoreaction. Analysis of the kinetic binding profile provided a fast and effective way to measure antigen concentration. Monitoring the immunoreaction between commercially available immunoglobulin $\mathrm{G}(\mathrm{IgG})$ and anti-IgG demonstrated the feasibility of using the MFPI sensing system for immunosensing applications. 


\section{Acknowledgements}

I would like to express the most sincere gratitude to my advisor Anbo Wang for giving me this opportunity to further my education. Also thanks for all his guidance, support during my doctoral studies, and for allowing me the freedom to pursue my own ideas and interests. I am deeply indebted to Drs. James R. Heflin, Ira Jacobs, Gary R. Pickrell and Ahmad Safaai-Jazi for their guidance, comments, encouragement and willingness to serve as my committee.

A special thank goes to Dr. Kristie L. Cooper for her assistance in technical writing, project management and many valuable discussions that facilitated my research. It is a great honor to work with you in the past years.

I would like to also extend my sincere thanks to all the faculty, staff and students in Center for Photonics Technology for their friendship, technical discussions, and support. Without their efforts and support, I would never have continued my academic development at Virginia Tech. The pleasure was mine to meet and work with these people.

Finally I would like to express my gratitude to my deepest love, Ying, for her help, support and encouragement in every aspect of my life; and to my parents and sister, who raised me and trust me with their endless love. 


\section{Table of Contents}

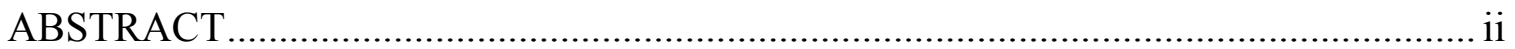

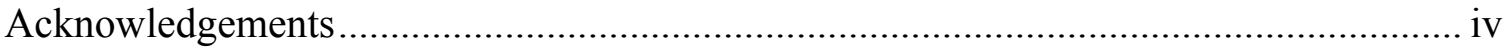

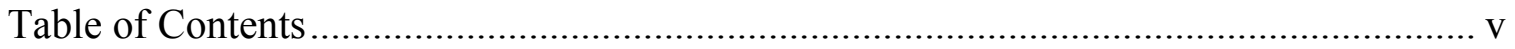

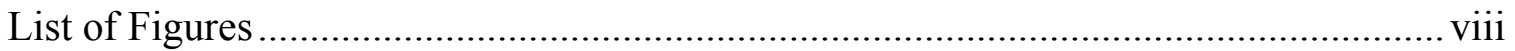

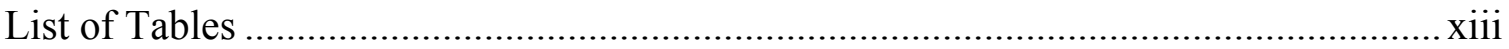

Chapter 1 Introduction ........................................................................................ 1

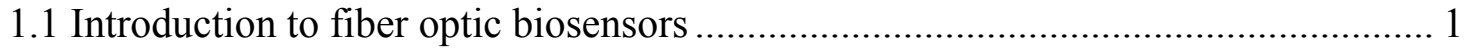

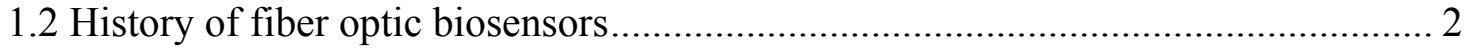

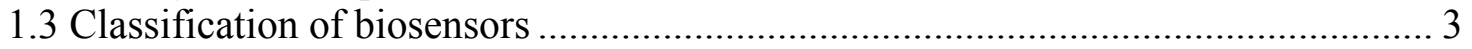

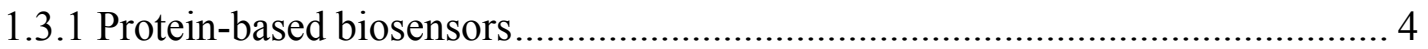

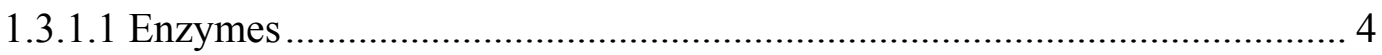

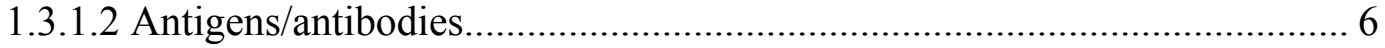

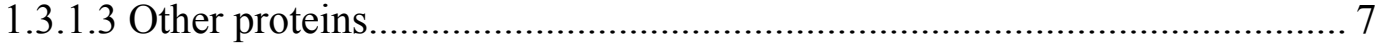

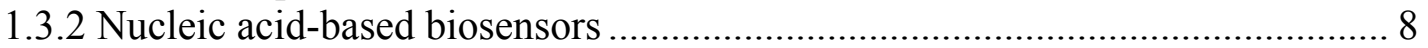

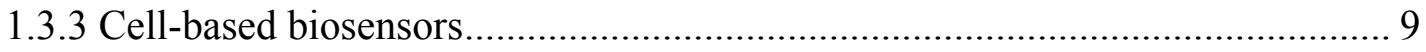

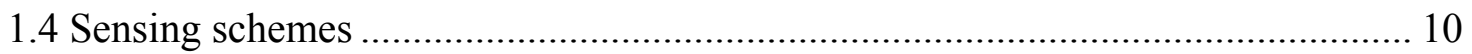

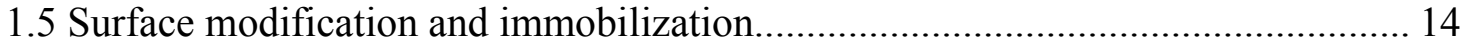

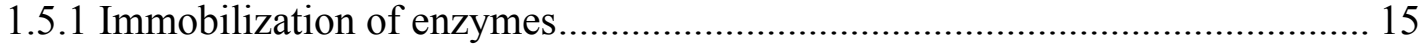

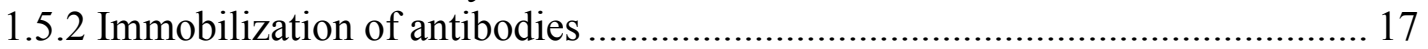

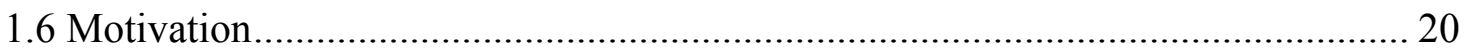

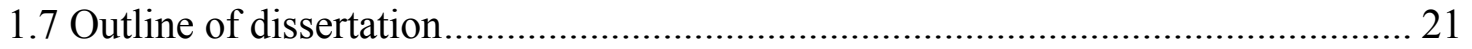

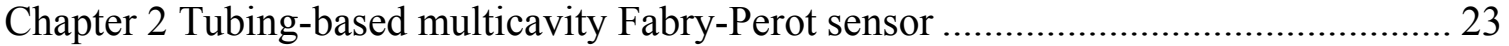

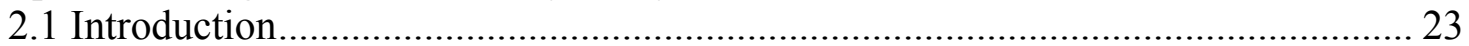

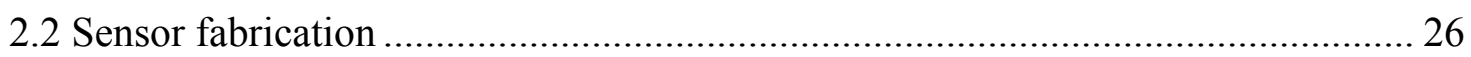

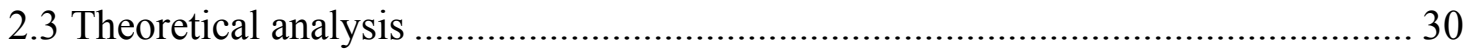

2.3.1 Conventional analysis for single-cavity Fabry-Perot sensor …...................... 30

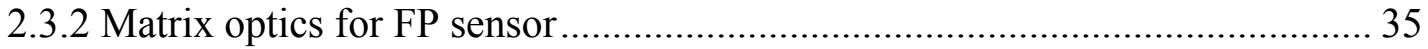

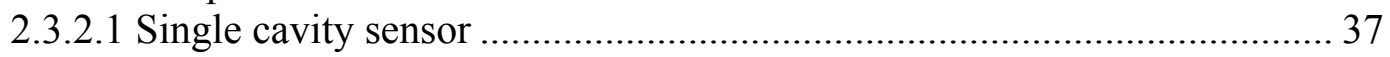

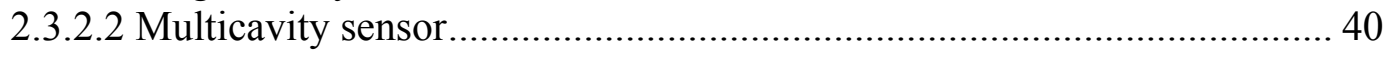

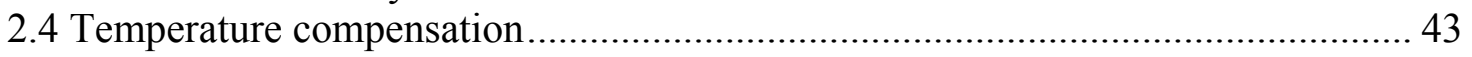

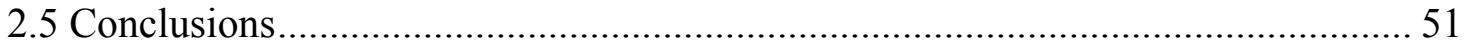

Chapter 3 Microgap multicavity Fabry-Perot sensor .............................................. 52

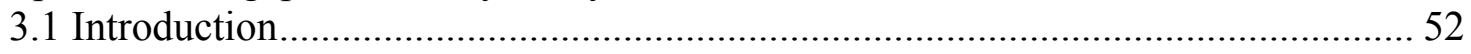




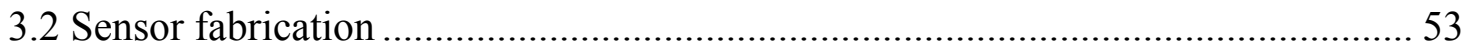

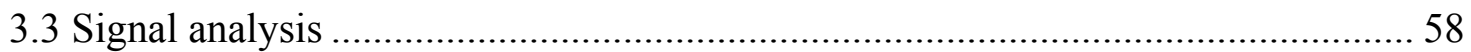

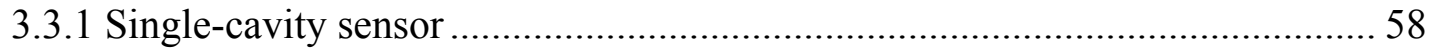

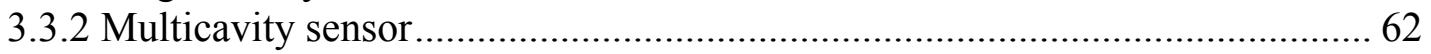

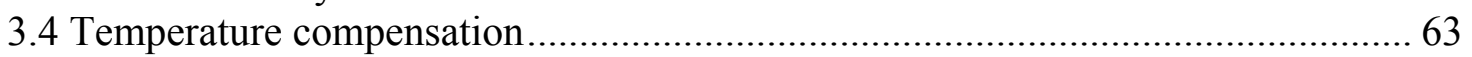

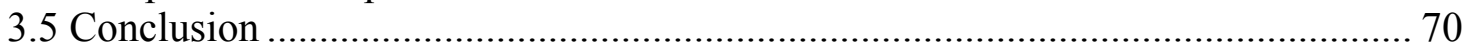

Chapter 4 Surface modification by polymer self-assembly ......................................... 72

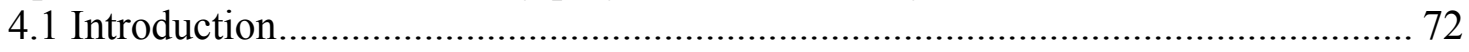

4.2 Characterization of polymer self-assembly by ellipsometry ………………......... 74

4.2.1 Analysis of factors on thin-film characteristics ……………........................... 74

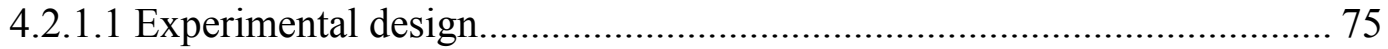

4.2.1.2 Materials and methods ................................................................... 76

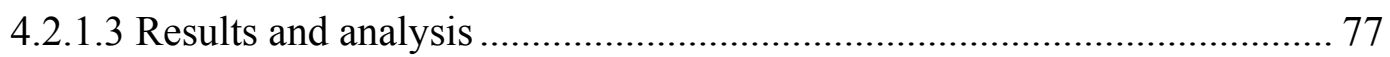

4.2.2 Stability of the polymer self-assembly ........................................................ 83

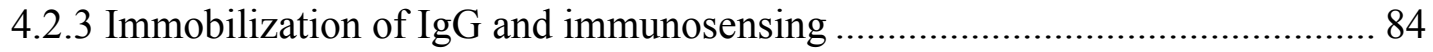

4.3 Thin film characterization by spectroscopy ………............................................. 85

4.4 Polyelectrolyte self-assembly on fiber and sensor.............................................. 90

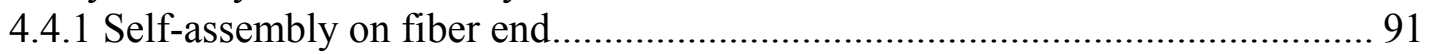

4.4.2 Self-assembly on multicavity sensors ........................................................... 95

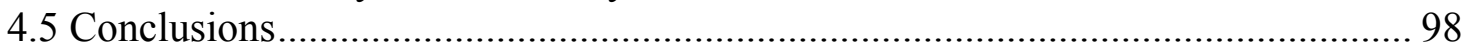

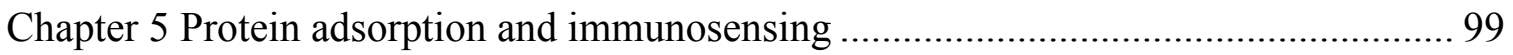

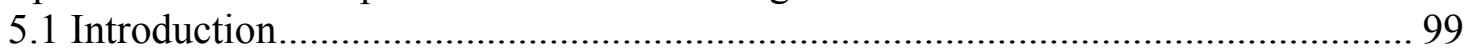

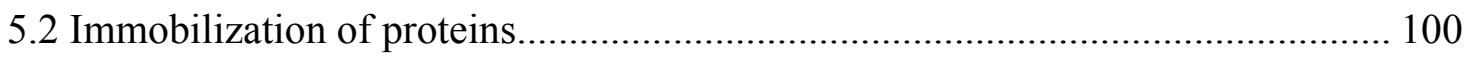

5.3 Antigen binding to immobilized monolayer IgG.................................................. 104

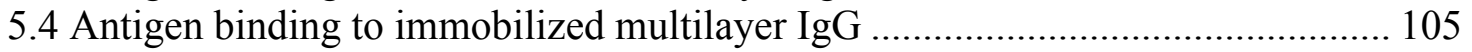

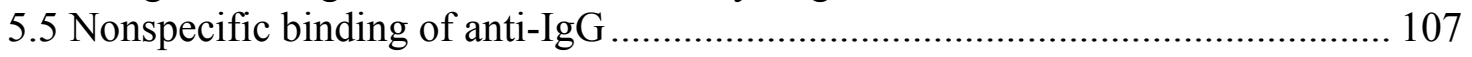

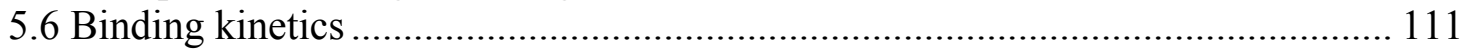

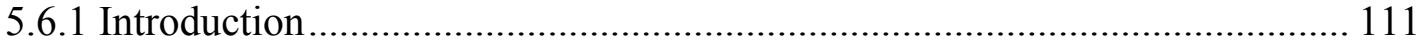

5.6.2 Theoretical model for kinetic measurement................................................. 113

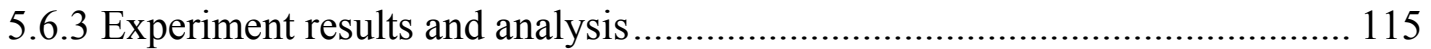

5.6.3.1 Antigen binding to immobilized $\mathrm{IgG}$..................................................... 115

5.6.3.2 Rate of change in optical thickness with antigen concentration .............. 118

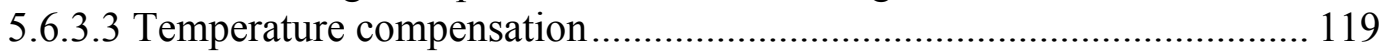

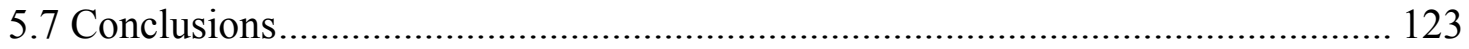

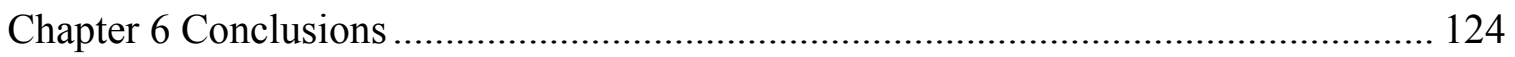

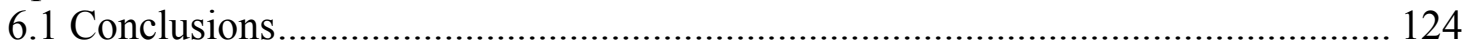

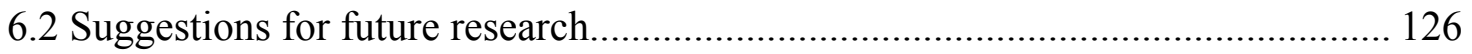

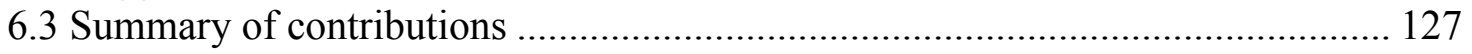

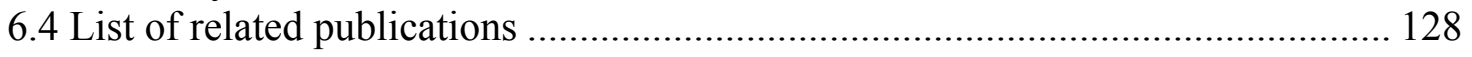

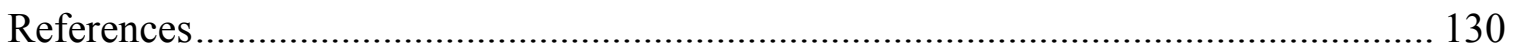




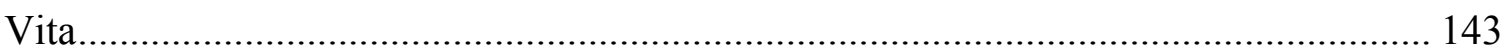




\section{List of Figures}

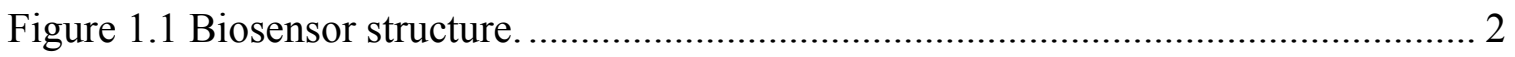

Figure 1.2 Assay format in immunosensors. (a) Direct assay (b) Competitive assay (c)

Binding inhibition assay (d) Sandwich assay ........................................................ 7

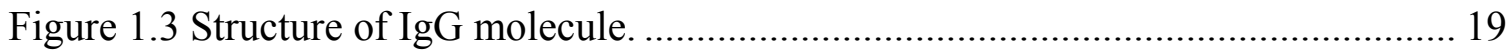

Figure 2.1 Structure of FFPI sensors. (a) FFPI sensor with silica tubing (ID>125 $\mu \mathrm{m}$ ) fabricated by laser bonding; (b) FFPI sensor with silica tubing ( $O D=125 \mu \mathrm{m})$ fabricated

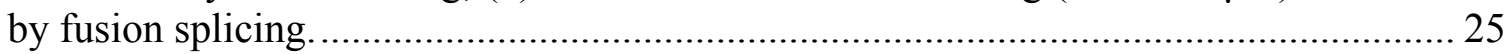

Figure 2.2 Structure of miniature FFPI sensors. (a) Diaphragm-based FFPI sensor; (b)

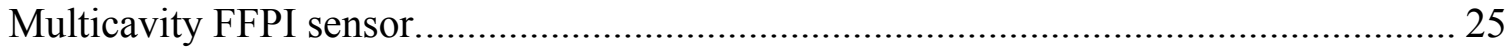

Figure 2.3 Procedures in fabrication of multicavity FFPI sensor. .............................. 27

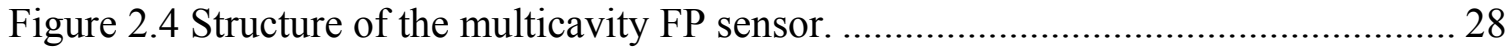

Figure 2.5 Picture of a tubing-based multicavity FP sensor (Zeiss Axiovert 25

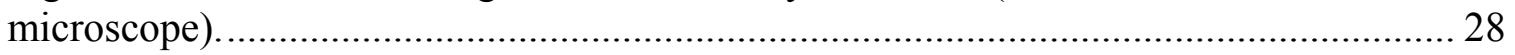

Figure 2.6 Typical reflection spectrum of a multicavity FP sensor.............................. 29

Figure 2.7 Structure of the low-finesse single-cavity FP sensor. ................................ 31

Figure 2.8 Coupling phase shift verses the cavity length........................................ 34

Figure 2.9 Power coupling coefficient verses the cavity length................................. 35

Figure 2.10 Notation for waves traveling over the interface and propagation inside the

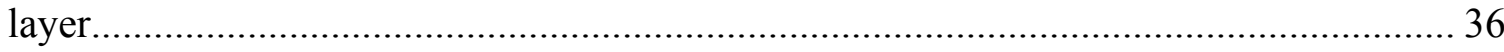

Figure 2.11 Comparison of the theoretical models for single FP cavity sensor. Here

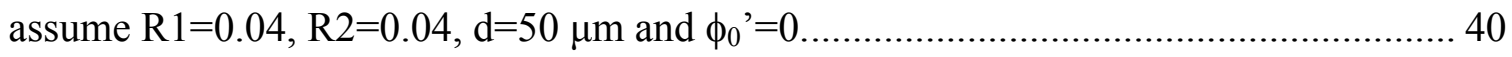

Figure 2.12 Comparison of the experimental data and theoretical fitting for a multicavity

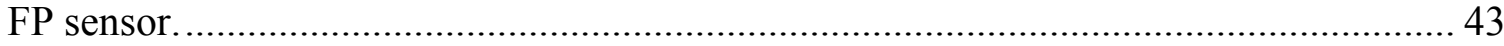

Figure 2.13 Reflection spectra of a multicavity FP sensor at $0^{\circ} \mathrm{C}$ and $100^{\circ} \mathrm{C} \ldots \ldots \ldots \ldots \ldots . . . . . . .44$

Figure 2.14 Schematic of multicavity FP sensor system for thin-film applications........ 46

Figure 2.15 Changes in air-cavity thickness with temperature from 0 to $100^{\circ} \mathrm{C} \ldots \ldots \ldots \ldots . . . . .47$ 
Figure 2.16 Changes in optical thickness of fiber cavity with temperature from 0 to $100^{\circ} \mathrm{C}$.

Figure 2.17 Comparison of changes in air-cavity thickness to the fiber-cavity optical thickness of an MFPI sensor with respect to temperature from 0 to $100^{\circ} \mathrm{C}$.

Figure 2.18 Effects of temperature compensation on the optic thickness error of fiber cavity in an MFPI sensor coated with thin film. The upper curve shows the optical thickness error before compensation in the temperature range from 0 to $100^{\circ} \mathrm{C}$. The lower curve shows the error after compensation.

Figure 3.1 The end face of a fiber (corning SMF-28) after 15 min etching in BHF....... 55

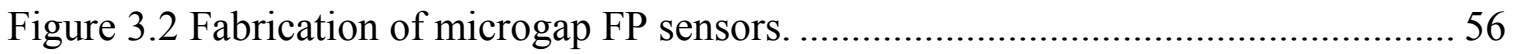

Figure 3.3 Picture of a microgap FP sensor............................................................ 56

Figure 3.4 Structure of the microgap multicavity FP sensor. .................................... 57

Figure 3.5 Picture of a microgap multicavity FP sensor........................................... 58

Figure 3.6 Reflection spectrum of a microgap FP sensor............................................ 59

Figure 3.7 Fourier transformation of the reflection spectrum..................................... 59

Figure 3.8 Fourier transformation of the bandpass-filtered spectrum. .......................... 60

Figure 3.9 Reflection spectrum of a microgap multicavity FP sensor........................... 62

Figure 3.10 Fourier transformation of the reflection spectrum of a microgap multicavity

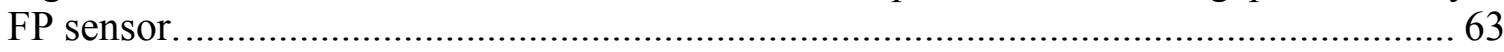

Figure 3.11 Schematic of microgap multicavity FP sensor system for thin-film

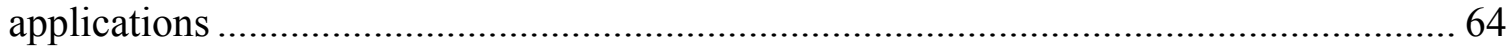

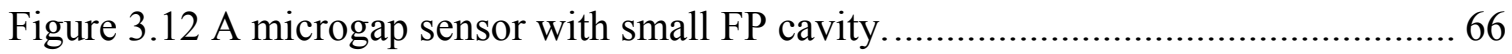

Figure 3.13 Changes in optical cavity length of temperature sensor with temperature from

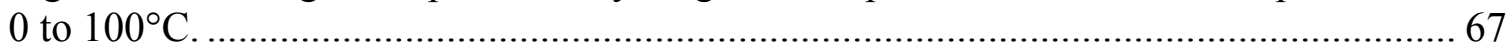

Figure 3.14 Changes in optical cavity length of thin-film sensor with temperature from 0 to $100^{\circ} \mathrm{C}$

Figure 3.15 Comparison of changes in optical cavity lengths of temperature sensor and thin-film sensor with respect to temperature from 0 to $100^{\circ} \mathrm{C}$. 
Figure 3.16 Effects of temperature compensation on the optical thickness error of thinfilm sensor. The temperature cycled twice by steps of $10^{\circ} \mathrm{C}$ in the range from 0 to $100^{\circ} \mathrm{C}$. The upper curve shows the optical thickness error before compensation and the lower curve shows the error after compensation.

Figure 3.17 Change in optical thickness of the $[\mathrm{PAH} / \mathrm{PSS}]_{10}$ film under temperature variations.

Figure 4.1 Adsorption steps of thin film deposition. The polycation adsorption and polyanion adsorption steps can be repeated to the required layer numbers. (a) Negative charged substrate; (b) Polycation adsorption; (c) Polyanion adsorption........................ 73

Figure 4.2 Main effects plot for $\mathrm{S} / \mathrm{N}$ ratios of thickness.......................................... 78

Figure 4.3 Main effects plot for means of thickness............................................... 79

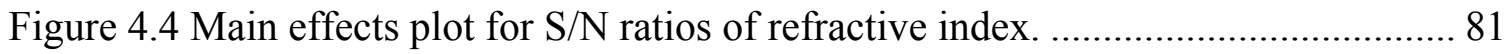

Figure 4.5 Main effects plot for means of refractive index.................................... 82

Figure 4.6 Variations in thickness and refractive index of $[\mathrm{PAH} / \mathrm{PSS}]_{10}$ film at room

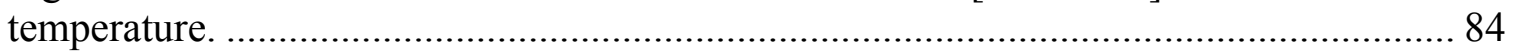

Figure 4.7 IgG immobilization on polymer film and immunosensing. ........................ 85

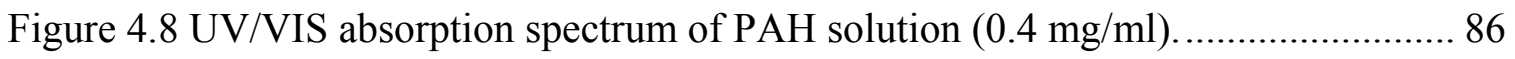

Figure 4.9 UV/VIS absorption spectrum of PSS solution $(0.04 \mathrm{mg} / \mathrm{ml})$....................... 87

Figure 4.10 UV/VIS absorption spectra of PAH/PSS multilayer film during the fabrication. 88

Figure 4.11 The absorbance of the PSS at $225 \mathrm{~nm}$ versus the bilayer number. 88

Figure 4.12 Comparison of absorption spectra of substrate with and without PAH/BSA film.

Figure 4.13 UV-VIS-NIR absorption spectrum of PAH/PSS multilayer film..... 90

Figure 4.14 Experimental setup for thin film FP cavity. 92

Figure 4.15 The reflection intensity changes with respect to the number of bilayers of PDDA/PS119. 93

Figure 4.16 The reflection intensity changes with respect to the number of bilayers of PAH/PSS. (a) $\lambda=1.3 \mu \mathrm{m}$;(b) $\lambda=1.52 \mu \mathrm{m}$. 
Figure 4.17 Optical thickness changes with the number of PAH/PSS layers on a tubingbased FP sensor

Figure 4.18 Optical thickness changes with the number of PAH/PSS layers on a microgap FP sensor.

Figure 5.1 Schematic of the multicavity FP sensor system.

Figure 5.2 Change in thickness after 60 min exposure of the polymer surface to pig IgG as a function of protein concentration. Standard errors are calculated based on multiple tests. Inset is the Scatchard plot which is a linearization of the Langmuir model.

Figure 5.3 Change in thickness after 60 min of exposure of the IgG to anti-IgG as a function of protein concentration $(n=1.432)$. Inset is the Scatchard plot which is a linearization of the Langmuir model

Figure 5.4 Change in optical thickness with the IgG/PSS multilayer formed on $[\mathrm{PAH} / \mathrm{PSS}]_{2}$ film.

Figure 5.5 Optical thickness changes in the fiber-film cavity during self-assembly of the polymer film, immobilization of sheep IgG and nonspecific binding with anti-rabbit IgG.

Figure 5.6 Optical thickness changes in the fiber-film cavity during self-assembly of the polymer film, immobilization of pig IgG, binding block, nonspecific binding with antirabbit $\mathrm{IgG}$ and immunosensing of anti-pig $\mathrm{IgG}$.

Figure 5.7 Optical thickness changes in the fiber-film cavity during self-assembly of the polymer film, immobilization of rabbit IgG, binding block, nonspecific binding with antipig IgG and immunosensing of anti-rabbit IgG.

Figure 5.8 Theoretical model for tubing-based multicavity sensor.

Figure 5.9 Changes in optical thickness during binding of anti-pig IgG at various concentrations $(\mu \mathrm{g} / \mathrm{ml})$ to immobilized pig IgG. 116

Figure 5.10 Theoretical fitting of binding anti-pig IgG $(20 \mu \mathrm{g} / \mathrm{ml})$ to immobilized pig IgG by Langmuir model.

Figure 5.11 Theoretical fitting of binding anti-pig $\operatorname{IgG}(20 \mu \mathrm{g} / \mathrm{ml})$ to immobilized pig IgG by empirical model.

Figure 5.12 Rate of change in optical thickness as a function of anti-pig IgG concentration for binding to pig IgG.

Figure 5.13 Schematic of microgap multicavity FP sensor system. 120 
Figure 5.14 Effects of temperature compensation on the change in optical thickness during immunosensing. The top curve shows the temperature changes during the test. The middle curve shows the change in optical thickness before compensation and the lower curve shows the change after compensation............................................................... 122 


\section{List of Tables}

Table 2.1 Comparison of the arc conditions (Sumitomo Type-36 Fusion Splicer) for multicavity sensor fabrication and normal SM fiber splicing.................................... 29

Table 4.1 Factors and levels for experimental design............................................ 75

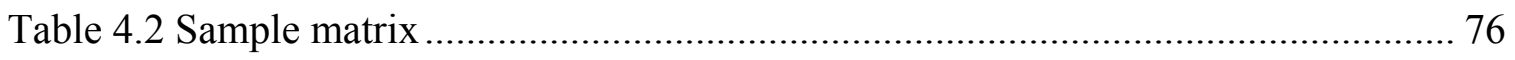

Table 4.3 Response table for signal to noise ratios (in $\mathrm{dB}$ ) of thickness........................ 80

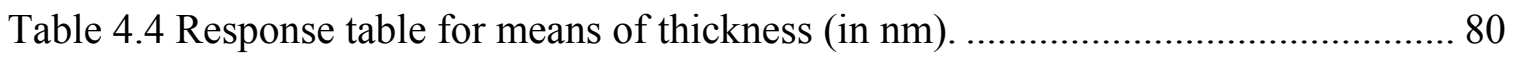

Table 4.5 Response table for signal to noise ratios (in $\mathrm{dB}$ ) of refractive index............... 82

Table 4.6 Response table for means of refractive index....................................... 82

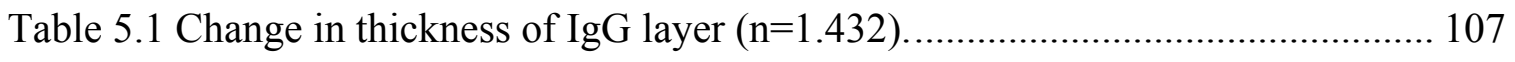




\section{Chapter 1 Introduction}

\subsection{Introduction to fiber optic biosensors}

Biosensors have attracted extraordinary interest in recent years, because of the increasing need for simple, rapid, and continuous in situ monitoring techniques in a broad range of areas. Biosensors can be regarded as an important alternative to conventional techniques due to their sensitivity, selectivity, versatility, promptness and capability in multianalyte monitoring.

According to the definition from International Union of Pure and Applied Chemistry (IUPAC), a biosensor is a self-contained integrated device providing quantitative or semi-quantitative analytical information which includes a biological sensing element in direct contract with a transducer [1]. The biological recognition elements, also called receptors, play the key role in the sensitivity and selectivity of sensing. The receptor translates biological information, such as an analyte concentration, into a chemical or physical output signal with a defined sensitivity. It provides the sensor with a high degree of selectivity for the analyte to be measured.

The receptor detects the analyte by a specific interaction with the target analyte, which will generate a physical or chemical perturbation that can be converted into a measurable effect such as an optical or electrical signal. The signal from the receptor is transmitted to the transducer which generates another signal that is proportional to the signal received. The key points in biosensing are the analyte recognition mechanism and the close connection between the sensing element and the transducer. The structure of a biosensor can be illustrated by Figure 1.1. 


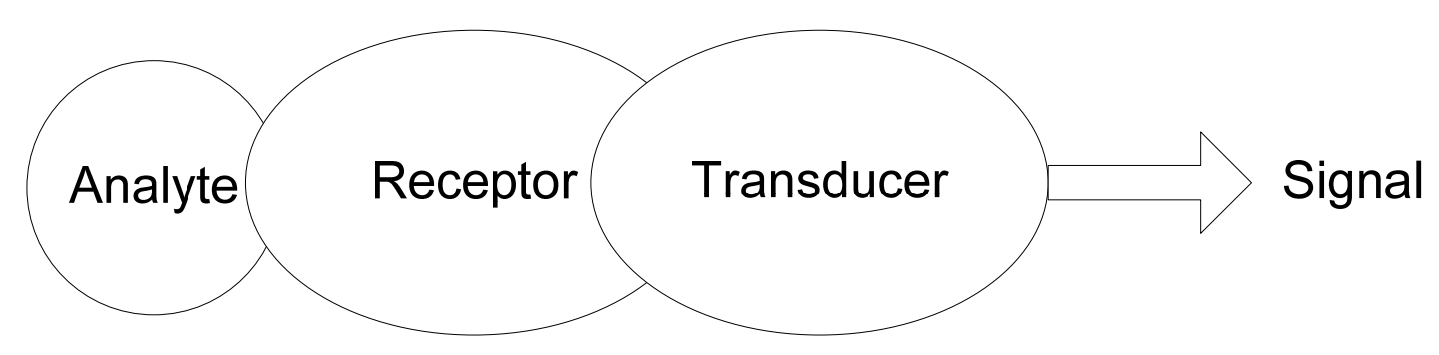

Figure 1.1 Biosensor structure.

The definition, classification and nomenclature of biosensors have been described in detail [1]. Biosensors may be classified according to the different transducer types, such as optical, electrochemical, piezoelectric and calorimetric. An optical biosensor is composed of a bioreceptor, an optical transducer, an excitation light source and a detector. The excellent selectivity of the bioreceptor and the outstanding sensitivity of optical detection methods make optical biosensors indispensable in biosensing.

Modern optical biosensors developed in conjunction with progress in the communication industry, information technology and molecular biology. Although different optical measurement techniques could be used in optical biosensing, optical fibers have been the key components in sensor development. In fiber optic biosensors, optical fibers act as the platform for biological recognition elements and medium for the excitation light and consequent signal. In fact, it is optical fiber that greatly improved the techniques for in situ and in vivo measurement. Optical fiber sensors succeed in situations where conventional sensors may not be appropriate. They are usually compact and lightweight, immune to electromagnetic interference, resistant to harsh environments and capable of multiplexing and remote sensing. The following sections will focus on the historical progress, classifications, principles and surface modification of fiber optic biosensors.

\subsection{History of fiber optic biosensors}

The development of optical fiber sensors is related to the two most important scientific advances of the 1960s [2], the laser [3] and modern low-loss optical fiber [4, 5]. 
The enormous improvement in transmissivity of optical fibers enabled long range communication and optical sensing over large distances. High quality fibers tremendously widened the field of optical sensing in the following years. During the early 1970s, an optical fiber probe was used in the chemical sensing [6]. The first optical fiber based chemical sensor was developed by Lubbers and Opitz in 1975 [7]. The sensor was designed to measure biological gases and fluids by fluorescence photometry. Soon after that, fiber optic biosensors were formed by coupling biological molecules to the chemical sensors. A glucose sensor was fabricated by coupling the enzyme glucose oxidase to an $\mathrm{O}_{2}$ optrode [8]. In the following years, biosensors with different recognition molecules were developed $[9,10]$. Although the basic configuration of the biosensor is similar, new types of optical fibers, optical instruments and biorecognition molecules have been integrated into the biosensors. New materials, combined with new immobilization techniques and advanced optical approaches, resulted in the development of more sophisticated selective and sensitive biosensors. Advances in new fiber optic technologies and molecular biology influence the development of biosensors. Integration of those technologies led to the evolution of biosensors.

\subsection{Classification of biosensors}

Biosensors can be classified according to the biological recognition mechanism. The recognition properties of the receptor can be classified as catalytic and affinitybased. Catalytic biosensors monitor the formation of a product, the disappearance of a reactant or the inhibition of the reaction in the biocatalyzed reaction. The biocatalyst can be enzymes, microorganisms, organelles or tissues. Typical products in catalytic reactions are low weight molecules which are preferred for electrochemical transducers. Chromogenic substances are required for optical transducers in order to determine the optical signals from the binding effect or optical labels from reactants. In affinity biosensors, the receptor molecules bind specifically to the analyte. Bioreceptors include antibodies, nucleic acids and hormone receptors. 
Based on the characteristics of receptors, the biosensor can be classified into three groups: protein-, nucleic acid- and cell-based biosensors. For example, immunoassay biosensors employ the specific binding between an antibody and antigen. Nucleic acid biosensors utilize the affinity of complementary single-stranded DNA (ssDNA) to form double-stranded DNA (dsDNA). Whole cell biosensors examine the effects of an analyte on an intact microorganism. The focus of this section is protein-based biosensors since they are the most well-studied and popular, and brief consideration is also given to studies on the other two kinds of biosensors.

\subsubsection{Protein-based biosensors}

Proteins are the most diverse of all macromolecules. There are thousands of different proteins, which perform a wide variety of functions. They serve as enzymes catalyzing nearly all the chemical reactions, antibodies providing a defense against infections, receptors essential for specific cell-cell interactions, and so on. Given the enormous complexity of proteins, several different classes of proteins have been used according to different requirements.

\subsubsection{Enzymes}

As the catalysts of biological systems, enzymes are extraordinary molecular devices that determine the patterns of chemical transformations and mediate the transformation of one form of energy into another. Enzymes regulate the rate at which chemical reactions proceed while not being consumed themselves. The most striking characteristics of enzymes are their catalytic power and specificity. Because of the high efficiency and specificity, as well as the broad range of analytes, enzymes are the most commonly used biological components of biosensors.

It is noteworthy that most enzymes do not change intrinsic optical properties when they interact with the analyte; therefore, to monitor the course of the enzymatic reaction, fiber-optic enzyme-based biosensors commonly operate in indirect or direct detection mode. For indirect detection, biosensors use an indicator or reactant to 
indirectly generate the optical signals. Direct detection means sensors detect the optical changes of the products, or intermediates, or cofactors including some inorganic ions and coenzymes such as nicotinamide adenine dinucleotide $\left(\mathrm{NAD}^{+}\right)$[11].

Oxidases, which catalyze the oxidation of compounds using oxygen, are usually co-immobilized with oxygen-sensitive luminescent materials, such as polycyclic aromatic hydrocarbons [12-14], porphyrins [15], and transition metal complexes [16-18]. Among them, the ruthenium diimine complexes, such as $\mathrm{Ru}(\mathrm{phen})_{3} \mathrm{Cl}_{2}$, and $\mathrm{Ru}(\mathrm{dpp})_{3} \mathrm{Cl}_{2}$ offer definite advantages including high photostability, long excited-state lifetimes, high quantum yields, and high quenching rate constants [19-21]. In 1996, Rosenweig and Kopelman developed the first micron-sized sensor for glucose by immobilizing glucose oxidase with $\mathrm{Ru}(\text { phen })_{3} \mathrm{Cl}_{2}$ in a polymeric support, and then attaching an acrylamide support to a silanized optical fiber tip surface by photoinitiated polymerization [22]. This sensor has a response time at least 25 times faster than larger sensors, and it shows as much as 5-6 orders of magnitude improvement in absolute sensitivity. Fiber optic biosensors have also been developed to measure serum concentrations of bilirubin [23], cholesterol [24] etc. by co-immobilizing the ruthenium diimine complexes with their specific enzymes.

Monitoring the $\mathrm{pH}$ change is another strategy of indirect detection, which has been successfully applied in measuring many metabolites, such as penicillin [25], creatinine [25], heavy metals [26] and chlorinated herbicide atrazine [27]. The enzymes immobilized in this type of biosensors are not restricted to one type; any enzyme that can produce acid or base to cause a localized $\mathrm{pH}$ change can be co-immobilized with $\mathrm{pH}$ sensitive chemicals.

In addition to single-enzyme fiber optic biosensors, dual- or tri-enzyme sensors have been developed in the last a few years. Multiple enzymatic sequential reaction can significantly improve both the sensitivity and detection limit of a sensor [28], or can be used to detect several related chemicals simultaneously [29]. 


\subsubsection{Antigens/antibodies}

An antigen is anything that makes the immune system respond to produce antibodies. Antigens could be living foreign organisms, such as viruses, bacteria, and also could be proteins, polysaccharides, lipids, or even dust. Antibodies are proteins that bind to the specific antigen with high affinity. By combining the inherent specificity of antigen-antibody reactions with the high sensitivity of various physical transducers, immunoassay biosensors are superior in sensitivity and selectivity.

There are four commonly used immunosensors: direct assay, competitive assay, binding inhibition assay, and sandwich assay (Figure 1.2). In a direct assay, the antigens are incubated with excess amounts of immobilized antibodies [30,31]. The antigen could be a fluorescent compound in fluorescence-based measurement [30]. A variety of techniques have been applied for unlabeled direct assay which include ellipsometry [32], optical waveguide lightmode spectroscopy [32], resonant mirror [33], surface plasmon resonance [34] and interferometry [35]. Unlabeled direct assay is a relatively quick and convenient method since it does not require the time-consuming and expensive labeling process. In order to use competitive assay, analytes or their analogs must be labeled with detectable chemicals, such as Cy5 and fluorescein (FITC) [36-38]. Upon incubating the analyte and labeled derivative will compete for limited binding sites of the antibodies. The amount of labeled derivative bound is inversely proportional to that of unlabeled analyte present in the sample. Thus the signal decreases as the analyte concentration increases. Binding inhibition assay is an alternative test format of competitive assay, with the antigens being immobilized and the antibodies being labeled $[39,40]$. In the sandwich assay [41-43], antibodies to analytes are first immobilized to a sensor. Next, the sensor is incubated with the analytes, and complexes are formed between the analytes and the immobilized antibodies. Finally, a secondary labeled antibody recognizes and binds to another epitope of the analytes. In this case, the extent of reaction is directly proportional to the amount of antigen present. The sandwich assay requires two antibodies that bind to epitopes that do not overlap each other on the antigen. This can be accomplished with either two monoclonal antibodies that recognize discrete sites or one batch of affinity- 
purified polyclonal antibodies. The cross reactivity is significantly reduced due to the recognition of different epitopes.

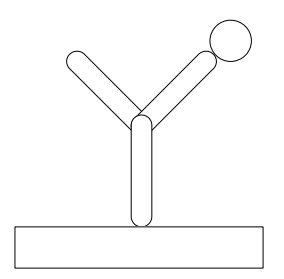

(a)

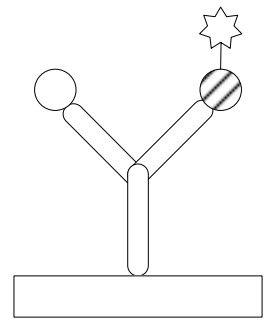

(b)

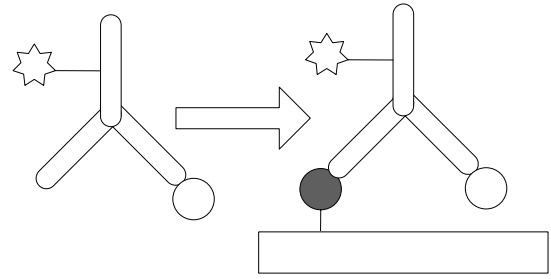

(c)

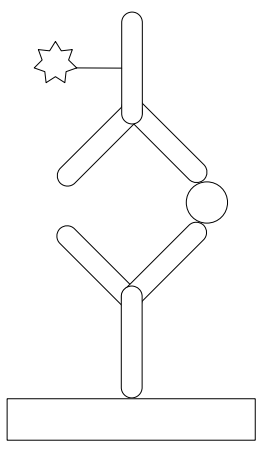

(d)

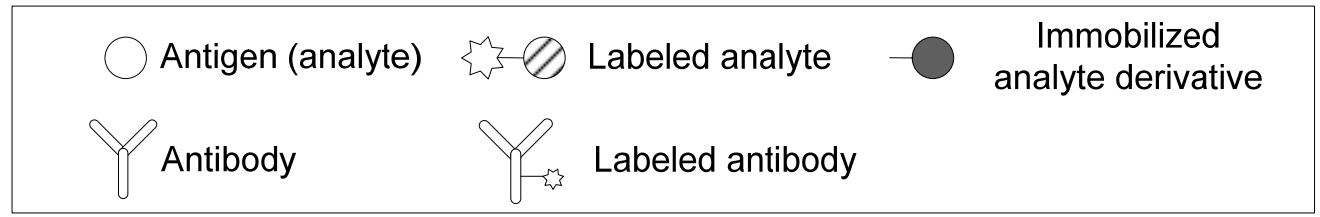

Figure 1.2 Assay format in immunosensors. (a) Direct assay (b) Competitive assay (c) Binding inhibition assay (d) Sandwich assay

The specific association of antigens and antibodies is dependent on hydrogen bonds, hydrophobic interactions, electrostatic forces, and van der Waals forces; therefore, the interactions between antibodies and antigens are reversible noncovalent interactions. They can be disrupted by high salt concentrations, extremes of $\mathrm{pH}$, and detergents. Several groups have shown the usage of chemical reagents to remove antigens bound to antibodies [44, 45], which makes it is possible to develop reusable, inexpensive, and reliable optical fiber biosensors.

\subsubsection{Other proteins}

Many other proteins in a cell, such as receptors and carrier proteins, are bound by a specific ligand. The interactions between them are strong and specific, which makes them excellent elements in biosensors. Based on this biorecognition principle, Barker et 
al. developed fiber optic nitric oxide-selective micro- and nanosensors using cytochrome $\mathrm{C}$ and guanylate cyclase as bioreceptors $[46,47]$.

\subsubsection{Nucleic acid-based biosensors}

With the development of the human genome project, more and more DNA sequences are available in the GenBank database. To date, the whole genome sequences of several species have become available. This provides the possibility to develop nucleic acid-based biosensors.

There are two types of nucleic acids: deoxyribonucleic acid (DNA) and ribonucleic acid (RNA). Since RNA is very easily degraded, most nucleic acid-based biosensors use DNA as the sensing agent. DNA molecules consist of two complementary strands, which are held together by hydrogen bonds formed between specific base pairs. The hydrogen bonds are weak enough to be reversibly broken under some situations, yet they are strong enough, when many form simultaneously, to help stabilize DNA. In DNA-based biosensors, single-stranded DNA is immobilized on the receptor, and then allowed to hybridize with labeled DNA complements. Recent studies [48-50] have also used intercalating dyes, such as thiazole orange (TO), ethidium bromide (EB) and SYBR as fluorescent dyes for detection. Those dyes cannot intercalate into ssDNA, and the emission of the dye is relatively low. Once double-stranded DNA forms, it undergoes a quantum yield enhancement. Thus, they offer a method for detection of hybridization without use of labeled DNA, and provide for an improved level of selectivity that is based on the structure of the hybrid rather than just the presence of DNA. Previous studies have not only tested the ability of DNA-based biosensors to detect synthesized oligonucleotides [51, 52], but also successfully used them in clinical and environmental analysis $[48,53]$.

DNA-based biosensors are extremely sensitive. Kleinjung et al. [54] showed that quantities as low as $30 \mathrm{fM}$ of analyte can be detected. If they are combined with other biological methods, such as polymerase chain reaction (PCR), even one molecule of analyte can be detected. They are also highly selective, since based on the characteristic of hydrogen bonds, only highly matched DNA can bind to the receptor and form double- 
stranded DNA. The lifetime of a biosensor is dependent on the stability of the adsorbed materials and the ability of regeneration; therefore, temperature and chemical sensitivity of proteins is an inevitable shortcoming of protein-based biosensors. In contrast, DNA is much more tolerant to temperature changes, and the hydrogen bonds between two strands can be broken just by heating at high temperature. Several studies $[48,52,55]$ have demonstrated that DNA-based sensors can be used for hundreds of cycles conducted over a span of several months without significant loss in binding activity.

\subsubsection{Cell-based biosensors}

In 2001, Ignatov et al. [56] developed a novel fiber optic biosensor for detection of lactate using cytoplasmic membranes (CPMs). CPMs from bacteria expressing lactate oxidase are adsorbed onto a cellulose disk, and the disk is fixed mechanically over an oxygen sensitive siloxane layer on the distal end of an optical fiber. This system can detect lactate with no interference from some other common substances, such as glucose, fructose or glutamic acid.

Whole cell cultured in vitro, such as algae cells [57], E. coli can also be used as biosensing elements. The genetically engineered $E$. coli contains an inducible promoter upstream of a reporter gene that encodes a bioluminescent protein. In the presence of a specific substance, the promoter is activated, and the bioluminescent protein is translated, and then it emits visible light. They have been applied for detecting heavy metals $[58,59]$ and toxic organic compounds $[60,61]$.

The biggest advantage of whole cell-based biosensors is they are more rapid in fabrication since they do not require lengthy preparation steps, such as extraction and purification. However, the cells or the organisms must be kept alive, so nutrients must be supplied and wastes removed. A biosensor immobilizing freeze-dried bacteria on the receptor, which has extended the storage lifetime of whole cells and increased portability somehow overcomes the shortcomings [62]. 


\subsection{Sensing schemes}

A biosensor is a combination of a biological receptor and a physicochemical transducer. The function of the transducer is not restricted to the substrate of the receptor but also to provide information on the structure and function of the receptor. The structure of the transducer is a closely related to the signal transduction principles. Transducers can be classified as electrochemical, optical, piezoelectric and calorimetric devices. Fiber optic biosensors have advantages over other techniques in terms of miniaturization, immunity to electromagnetic interference and resistance to harsh environment. Capability for multiplexing and remote sensing also makes fiber optic sensors superior to other competitors. The conventional technologies involved in fiber optic biosensor development include absorbance, reflectance, fluorescence and luminescence.

The absorbance of the analyte at a specific wavelength can be applied to determine the analyte concentration. Light is transmitted to the sample through an optical fiber and the absorbance is detected through a second fiber. Many reaction products or intermediates are colored [63]. When a product is colorless, an indicator can interact with it to produce a change in color [64]. The colored product can also be detected by reflectance with a bifurcated optical fiber [65]. Anomalous reflection (AR) refers to the decrease in reflectivity of a gold surface under blue or violet light due to the adsorption of a surface layer, which is applicable to affinity biosensors based on fiber optics [66]. Colorimetric resonant reflection can be applied as a direct biochemical assay technique [67]. A colorimetric resonant diffractive grating surface is used as a surface binding platform. The reflected wavelength is shifted due to the change of the optical path. Therefore by immobilizing the receptor to the grating surface, no label is required for the detection of analytes.

Fluorescence measurement is another widely used method for optical biosensors. Most fluorescence techniques employ a fluorescent dye to indirectly monitor the reactions, since the fluorescence is always affected by the presence of another product. For instance, a $\mathrm{pH}$-sensitive fluorescent dye is sensitive to an acidic product [68]. The 
decrease in fluorescence intensity of the dye seminaphthofluorescein (SNAFL) caused by liberated $\mathrm{NH}_{3}$, a product of the L-glutamate and L-glutamate oxidase reaction, was examined in study of L-glutamate [69]. Quantum dots (QDs) were used for in vitro assay detection of DNA, proteins, and other biomolecules [70-72]. QDs can be excited at almost any wavelength below the band edge and exhibit high photobleaching resistance and multiplexing capabilities [73]. The unique features of QDs make them more and more attractive in biological applications.

Chemiluminescence (CL) refers to the emission of light from a chemical reaction. Electrochemiluminescence (ECL) is the luminescence generated during an electrochemically initiated reaction. CL and ECL methods are commonly used for detecting hydrogen peroxide from enzymatic reactions. Since no excitation light source is required, the experiment setup is simplified and no extra instrument is needed for removing scattered excitation light. The selectivity and sensitivity are improved over conventional techniques such as absorbance, reflectance and fluorescence [74]. A fiber optic biosensor was developed to monitor the enhancement of chemiluminescence due to the addition of enhancers [75]. In the ECL technique, an electrode is required to electrochemically trigger CL. The electrode is usually placed at the distal end of a fiber, which is used to deliver the signal to a detector. Glucose can be detected by ECL of luminol, which reacts with hydrogen peroxide generated from the reaction of glucose and glucose oxidase [76]. Marquette et al. also employed luminol-peroxide ECL for flow injection analysis of glucose, lactate, cholesterol and choline [77].

More and more new fiber optic biosensing techniques have been proposed with promising futures. In addition to the plain waveguide functions, optical fibers have been used in surface plasmon resonance (SPR), Raman scattering, imaging and sensor arrays, gratings and interferometry.

Surface plasmon resonance refers to the optical excitation of surface plasmons at the metal-dielectric interface. The fields associated with the surface plasmon extend into the adjacent media with exponential decay. Therefore the surface plasmon is sensitive to changes near the interface. SPR biosensors are sensitive to changes near the sensor 
surface therefore eliminate the need for labels [78]. Fiber optic SPR biosensors have inherent advantages over conventional SPR techniques such as miniaturization, robustness, and remote sensing capability [79-81]. The sensing elements in fiber optic SPR sensor can be a chemically etched fiber containing a conical core [80], side-polished fiber [79], or various probe geometries [82].

The high sensitivity of SPR is due to the enhancement of the field at the metaldielectric interface. Field enhancement can also occur on metal nanoparticles such as gold and silver, which is named as localized surface plasmon resonance (LSPR). The resonance frequency of the LSPR is highly dependent upon the local environment of the particles [83]. A fiber optic LSPR biosensor was fabricated by depositing self-assembled gold colloids on the uncovered fiber core [84]. Another highly sensitive LSPR biosensor was fabricated by adsorbing gold nanoparticles at the endface of the fiber [85]. LSPR simplified sensor fabrication but maintained the advantages of high sensitivity and labelfree measurement.

Surface enhanced Raman scattering (SERS) has been utilized in biological and chemical applications [86, 87]. Silver-coated etched fiber tips with submicron diameters were used as the SERS substrate. It is possible to position the tip and the exciting laser to designated areas of the biological samples, thus enabling the recording of the local spectra with high spatial resolution.

Optical fiber microwell/microbead arrays have been applied to nucleic acid biosensing [88]. The microspheres were encoded with a fluorescent dye, functionalized with probe oligonucleotides and immobilized in microwells of etched imaging fiber. The microarray with multiple microspheres increases the signal to noise ratio and enhances the detection capabilities. In a whole cell biosensor, the fiber optic array enabled the simultaneous monitoring and spatially resolving the fluorescence signals obtained from individual cells [89]. Cellular responses of the cells were monitored by reporter genes or fluorescent indicators.

Fiber gratings consist of a periodic perturbation in the optical fiber. The classification of fiber gratings is generally based on the period of the gratings. Fiber 
Bragg gratings (FBG) have a submicron period and couple light from the propagating mode to the counterpropagating mode at a specific wavelength, which is sensitive to the temperature and strain [90]. Thinned fiber Bragg gratings (FBG) have been proposed as high sensitivity refractive index sensors, combined with easy multiplexing capability [91]. Simultaneous measurement of refractive index and temperature was demonstrated on a single nonuniform thinned grating, where the cladding layer was removed in part of the grating [92]. The same approach can be used as a key element in chemical and biological sensing in practical applications. The long period grating (LPG) has a period of $100 \mu \mathrm{m}$ to $1 \mathrm{~mm}$ and couples the propagating core mode and co-propagating cladding mode. The center wavelengths of the attenuation bands are sensitive to the local environment such as temperature, strain, bend radius and the refractive index of the medium surrounding the fiber [93]. LPGs can be employed in biosensing as the refractive index in the evanescent field changes upon the biological interactions [94]. Furthermore, no label is required for the analyte as a result of changes in refractive index.

Optical interferometry compares differences experienced by two light beams traveling along similar paths. A Mach-Zehnder interferometer has been applied in biosensing on planar waveguides [95, 96] or optical fibers [97]. A Fabry-Perot interferometric approach was utilized on a porous silicon substrate [98]. The diverse properties of porous silicon, including the refractive index [98, 99], photoluminescence $[100,101]$ and electrical properties [102], attract a great deal of interest in investigating the material as the transducer for a sensing system. Porous silicon displays well-resolved Fabry-Perot fringes in its reflection spectrum that are related to the effective optical thickness of the layer. The refractive index change with the binding of molecules thus shifts the reflection spectrum, which was demonstrated for DNA and proteins [98]. Dancil et al. utilized porous silicon as an immobilization and transducing matrix for protein-protein binding by monitoring the change in effective optical thickness from the Fabry-Perot fringes [103].

Spectral interferometry has been used as an feasible approach to detect changes in optical path length in thin films from immunoreactions [104]. Thin films were fixed to a 
glass substrate which located at the end of a bifurcated optical fiber. Electrostatic selfassembly was applied in fabrication of a Fabry-Perot cavity at the end of fiber [105]. A fiber optic humidity sensor was designed based on the FP cavity since the reflection intensity varies with the reflection coefficient of the external mirror [106]. The reflection spectrum is also sensitive to the absorbance of the cavity materials, which can be applied in chemical sensing [107]. However, high quality FP cavity was difficult to achieve by the self-assembly alone. Tens or hundreds of layers of self-assembly was required to form the cavity since each layer is only a few nanometers. The fringe visibility decreased as the film became thicker due to the absorption and scattering. Furthermore, the thickness resolution is limited by the intensity-based measurement. In another approach, Lin et al. reported the fabrication of a micro FP cavity by self-assembled polymer films on optical fibers, which was applicable for immunosensing [108]. But gold interface layers were needed to increase the reflection intensity and the cavity shape was not well controlled.

\subsection{Surface modification and immobilization}

The performance of a biosensor is closely related to the surface coverage of the biosensing molecules. The quality of the receptor layer also depends on mobility, accessibility and structure of the immobilized elements. Biological molecules are highly efficient at recognizing specific analytes or catalyzing reactions, but only in specific aqueous media, which limits their applications in biosensor development. The lack of preferred medium often results in denaturation and loss of reactivity. Immobilization techniques that can stabilize and preserve the receptor reactivity are actively pursued in the biosensing field.

Fiber optic biosensors require immobilization of biological recognition elements either to the substrate when fibers are used simply as a waveguide [24, 26, 27] or directly to the optical fiber serving as a sensing element [22, 23, 25, 109]. As introduced in the classification of biosensors, biological receptors include proteins, nucleic acids, organelles and cells. The focus of this section is the surface modification techniques for protein immobilization since they are the most popular receptors. In addition, only direct 
immobilization of receptors onto the surface of the transducer will be discussed, which is more attractive due to the compact structure and more convenient for in situ applications. Immobilization of biomolecules is one of the critical issues in development of fiber optic biosensors. The technique requires stable and effective immobilization of biomolecules onto a fiber surface while maintaining their recognition capabilities. Many methods have been developed for immobilization of biomolecules onto an optical fiber such as physical adsorption, covalent attachment, biomolecular interactions and host matrix encapsulation.

\subsubsection{Immobilization of enzymes}

Enzymes are widely used as biological recognition elements. Immobilization of enzymes greatly affects the reliability and performance of a biosensor. The maintenance of catalytic activity and the mechanism of signal transduction are the major concerns in the selection of the immobilization method. Chemical immobilization of the enzymes involves the formation of covalent bonds between enzymes and the substrate which requires specific functionalities on the biomolecules. Glutamate dehydrogenase (GDH) has been directly immobilized onto an optical fiber by covalent binding mechanism for glutamate detection [11]. The optical fiber surface is initially activated by silanization, and then functional groups affixed by cross-linking. The GDH molecules are able to attach to the functional groups on the fiber surface. A multilayer enzyme assembly of alkaline phosphatase (AP) was immobilized on an optical fiber by chemical cross-linking in the detection of organophosphorus pesticides [110]. Enzyme layers were covalently linked by a bifunctional amino coupling reagent, bis(sulfosuccinimidyl) suberate (BS3).

Another method for preparing enzyme-based fiber optic biosensors was developed by avidin-biotin coupling [111]. The silanized fiber was first coated with a polymer layer containing pendant amino groups that could react with succinimidylderivatized biotin. Then biotin-labeled enzymes were introduced to the fiber through a sandwich biotin-avidin-biotin interaction.

Recent research has demonstrated that enzymes can be immobilized in a host matrix. In contrast to the covalent attachment, the encapsulation of enzymes in the matrix 
does not significantly decrease the affinity and activity of the molecules; moreover, it maintains the stability, reactivity and homogeneity due to the noninvasive interactions. A suitable host matrix should be compatible with different reagents, transparent at the analytical wavelengths, stable under variable conditions, highly permeable to the analyte, and easily incorporated into the sensor. Traditional biosensor membrane matrices such as carbon materials, acetate polymers and gold layers are not suitable for direct spectroscopic studies due to the high extinction coefficients in the IR range. Sol-gel, a novel class of materials, can be a promising host matrix in optical sensing due to its chemical inertness, thermal stability and transparency [112]. The physical and chemical properties of sol-gel can be well controlled by adjusting the constituents. Furthermore, the mild formation condition is suitable for protein immobilization [113]. In fiber optic biosensors, the sol-gel with immobilized enzymes may be attached to the fiber end directly [114].

In addition to the sol-gel bioencapsulation, various polymer matrices have been used to immobilize proteins such as epoxy-amine resins, polyvinyl plastics, polyurethane foams and silicone elastomers [115]. In fiber optic enzyme-based biosensors, enzymes or indicators can be immobilized in the polymer support, which is attached covalently to a silanized optical fiber surface. Rosenzweig developed a glucose sensor where glucose oxidase was immobilized in an acrylic polymeric support. The acrylamide support was covalently attached to a silanized fiber by photopolymerization [22]. A similar immobilization method was applied to a fiber optic sensor for analysis of bilirubin [23]. Bilirubin oxidase was physically immobilized in a polymer matrix that is attached to a silanized fiber via photopolymerization. Polyvinyl alcohol (PVOH) has been explored as a substrate for immobilizing indicators in fiber optic chemical sensors [109]. Muller et al. immobilize corresponding enzyme layers in the $\mathrm{PVOH}$ matrix of $\mathrm{pH}$ optode for detection of penicillin, urea, glucose and creatinine [25]. 


\subsubsection{Immobilization of antibodies}

Immunosensors are based on selective bioaffinity interactions between antibodies and antigens. Covalent immobilization of antibodies was developed using thiol-terminal silanes and heterobifunctional crosslinkers [116]. This process and modified protocols are widely adopted in fiber optic immunosensors [37, 117-119]. A fiber optic immunosensor for cocaine detection was developed by covalently immobilized the monoclonal antibody on silanized fibers [37]. In a multichannel fiber-optic immunosensor for detection of explosives, antibodies were crosslinked to the silanized fiber with active thiol groups [117]. Brummel et al. developed a fiber optic immunosensor for detection of cyclodiene insecticides by covalently binding antibodies on quartz fibers [118]. Before immobilization of the antibody, the fiber was first silanized to introduce active thiol groups, and then reacted with cross-linking reagent. Cullum et al. utilized submicron fiber optic immunosensors to measure toxic chemicals in single cells. Fiber tips were coated with silver, then silanized and cross linked with antibodies [119].

Antibodies or antigens can be immobilized on the fiber by means of a biotinstreptavidin-biotin bridge [120]. The fiber was first silanized, and then incubated in a succinimidyl-derivatized biotin solution. Next, the fiber was transferred to a streptavidin solution. The resulting fiber can be used to immobilize any biotinylated antibody or antigen. In a similar scheme, antibody was immobilized on an optical fiber by means of an avidin-biotin bridge. Avidin was immobilized on the tapered fibers by the heterobifunctional crosslinker. The captured antigen was then detected by a fluorescently labeled secondary antibody [121]. Polymeric biotinylated thin film on optical fibers can facilitate the links to avidin and further biotinylated antigens or antibodies [122]. Biotinavidin/streptavidin technique provides a specific and strong non-covalent binding and a passivation layer over the substrate to prevent non-specific adsorptions.

The sol-gel process also provides a suitable host matrix for antibody immobilization. Fluorescein-labeled D dimer antibodies were immobilized on the optical fiber tip by the sol-gel technique [123]. Sol-gel encapsulation not only retains the activity 
of the protein by maintaining an aqueous environment around the protein but also enhances the sensitivity and selectivity with high surface areas and optimal pore sizes.

Antibodies can directly adsorbed onto the substrate by hydrophobic and electrostatic interactions $[124,125]$. Hydrophobic interactions refer to the tendency of hydrocarbons to form intermolecular aggregates in an aqueous medium, which appears as the dehydration of the protein and substrate, and leads to conformational changes of immobilized molecules. Hydrophobic interactions are influenced by the hydrophobicity of the surface. Electrostatic interactions vary with the surface charge, isoelectric point of proteins, $\mathrm{pH}$ and ionic strength of the solution [126].

Immunoglobulin $\mathrm{G}(\mathrm{IgG})$ as one of the major antibodies is commonly applied in immunosensing. Each IgG molecule consists of four polypeptides which two heavy chains and two light chains joined to form a "Y" shaped molecule (Figure 1.3). IgG molecules have three protein regions. Two arms of the "Y" $\left(\mathrm{F}(\mathrm{ab})_{2}\right)$ are receptor sites that recognize the specific partner. The third region $(\mathrm{Fc})$ forms the base of the " $\mathrm{Y}$ " and it is important in certain aspects of immune response [127]. The adsorption of IgG molecules and their $\mathrm{F}\left(\mathrm{ab}^{\prime}\right)_{2}$ fragments on hydrophilic surfaces are retarded when repulsive. This effect is stronger for $\left.\mathrm{F}(\mathrm{ab})_{2}\right)_{2}$ than for the whole IgG molecule, whereas Fc adsorption is not significantly affected [126]. Therefore the whole IgG molecule tends to adsorb by its Fc fragment in an end-on orientation which is preferred for antigen binding. However, the adsorption of IgG by its Fc fragment is relatively weak under conditions of low ionic strength. Proteins are more tightly bound to hydrophobic surface than hydrophilic surface [126]. By surface modification techniques such as polyelectrolyte self-assembly, a close-packed IgG layer could be produced which is less time-consuming, cost-effective and simpler than other immobilization techniques. 


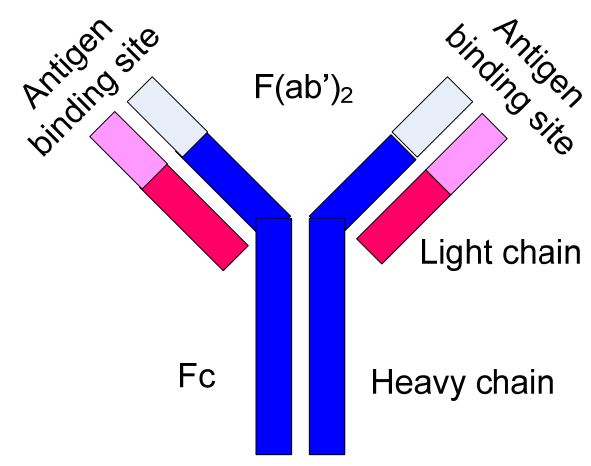

Figure 1.3 Structure of IgG molecule.

In fiber optic biosensors, proteins such as enzymes or antibodies immobilized directly on a solid substrate or encapsulated in a porous matrix are used to detect the presence of a target substrate or antigen. In general, immobilized proteins exhibit lower biological activity compared to those in aqueous media, which may be due to blocking of the receptor's binding sites. In addition, immobilization leads to heterogeneity of the protein activity due to steric effects and conformational changes. Antibodies, enzymes and other receptors exhibit uniform kinetic rate constants and binding affinities. But nonuniform kinetic and thermodynamic properties appear after immobilization. Vijayendran et al. compared the binding heterogeneity of the analyte with antibodies immobilized by different strategies [128]. Optical fibers were used as the substrate for both random and structured immobilization schemes. Binding affinities of immobilized antibodies were at least an order of magnitude lower than the solution value. Random immobilized antibodies displayed heterogeneous binding characteristics, while more homogeneous protein preparations exhibited more uniform behavior. The decreased biological activity and heterogeneity limit the sensitivity and reproducibility of biosensors. Therefore, considerable interest is focused on increasing the biological activity and eliminating the heterogeneity associated with immobilized proteins [129, 130]. Surface modification techniques are essential for efficient adsorption of biological molecules onto optical fibers. Oriented and site specific immobilization of biological compounds are still in progress. 


\subsection{Motivation}

The growing need for real-time and low-cost technology in clinical diagnosis, pharmaceutical and drug analysis, pollution control and monitoring urges the development of various biosensors. Fiber optic sensors succeed in situations where conventional sensors may not be appropriate. They are usually compact and lightweight, immune to electromagnetic interference, resistant to harsh environments and capable of multiplexing and remote sensing.

Direct immobilization of receptors onto optical fibers is more attractive due to the compact structure and more convenient for in situ applications. However, proteins are denatured when directly immobilized onto the optical fibers [131]. Surface modification is required to immobilize proteins and preserve their reactivity. Polyelectrolyte selfassembly is a simple but efficient method for supporting or embedding receptors. Furthermore, it can increase the capacity of receptors through the fabrication of a multilayer film.

External reagents are usually required in biosensing such as labeled antigens in a competitive assay (Figure 1.2 (b)) and secondary antibodies in a sandwich assay (Figure $1.2(\mathrm{~d})$ ). This procedure complicates the analysis and limits the biosensors as simple analytical tools. Possible photobleaching and wash out of indicators further restrict their applicability. The fabrication of a reagentless biosensor attracts more and more attention. The direct sensing scheme is to measure the intrinsic optical properties of the analyte, such as the refractive index, thickness or absorption. The absorption peak wavelengths of biomolecules such as proteins and DNA are normally in the UV range (260-280 nm) which is not suitable for transmission in optical fibers [132]. Direct measurement of the optical thickness, i.e. the product of refractive index and thickness, of the adsorbed analyte can be achieved by Fabry-Perot interferometry. It simplifies the probe fabrication compared to other techniques such as SPR, SERS and gratings, yet achieves high sensitivity in monitoring the immobilization and binding process due to the inherent nature of interferometry. 
Fiber optic Fabry-Perot interferometric (FFPI) sensor is distinguished for its multiplexing capability. Thus it provides the possibility to examine multiple factors simultaneously. Temperature-induced errors of fiber optic sensors greatly affect the accuracy in measurements. Therefore it is possible to compensate the temperature dependence by extracting the temperature information from a second multiplexed sensor. This provides the opportunity to examine the binding process and thin-film characteristics under different environment temperatures.

\subsection{Outline of dissertation}

In this dissertation, the first chapter gives a brief overview on the fiber optic biosensors, including the historical view, classification, sensing schemes, surface modification and motivation.

Chapter 2 describes the fabrication and theoretical analysis of a tubing-based multicavity FP sensor. The sensor is not only applicable to the measurement of polyelectrolyte self-assembly but also to the monitoring of immobilization and binding

process of immunosensing. The temperature dependence in thin-film measurement can be compensated by extracting the temperature information from the multiplexed cavity.

Chapter 3 describes the fabrication and analysis of a microgap multicavity FP sensor. It not only has the advantages similar to the tubing-based sensor but also excels at its simplicity in fabrication process and flexibility in cavity lengths. The improvement on the temperature compensation is discussed.

Chapter 4 proposes polyelectrolyte self-assembly as the surface modification method for immobilization of biological receptors onto the fiber optic sensor. The multilayer buildup was examined by ellipsometry and absorption spectroscopy. Factors that affect characterization of the polymer self-assembly are systematically investigated. The interferometric method demonstrates higher sensitivity than intensity-based measurement in fiber optic thin-film sensing.

Chapter 5 introduces immobilization of antibodies onto multicavity FP sensors coated with polyelectrolyte self-assembly. The thickness changes in high concentrations 
of IgG are closed to the thickness of a monolayer. The feasibility of using the multicavity FP sensor as a biosensor has been verified. Analysis of the binding kinetics provides a fast and effective way in measurement of the antigen concentration.

The last chapter, Chapter 6, summarizes the major contributions and gives some suggestions for future improvement of the multicavity sensors in biosensing. 


\section{Chapter 2 Tubing-based multicavity Fabry-Perot sensor}

\subsection{Introduction}

The sensing industry involves very diverse technologies and various applications. Depending on the requirements of the application, different technologies could be used to meet those needs. In fiber optic sensing, technologies include fiber gratings, interferometers, fluorescence and others. As one of the major technologies, fiber-optic Fabry-Perot interferometric (FFPI) sensors have been widely used in areas such as temperature, pressure, strain and flow sensors [133-141]. The advantages of FFPI sensors include high sensitivity, ease of fabrication, localization and lead insensitivity since light is transmitted to and from the sensor through the same fiber. Based on phase modulation, FFPI sensors are immune to optical power fluctuations. Fringe patterns from a FabryPerot (FP) cavity are modulated by environmental factors such as temperature, pressure and others. Monitoring the changes in the optical cavity allows measurands to be investigated with high accuracy and resolution. With white-light interferometry, FFPI sensors can achieve high resolution and large dynamic range together with absolute measurement [142].

Fabry-Perot interferometers have been extensively deployed in a variety of fiber optic configurations due to the relative ease of fabrication. FFPI sensors can be classified as intrinsic Fabry-Perot interferometric (IFPI) sensors and extrinsic Fabry-Perot interferometric (EFPI) sensors. In IFPI sensors, the sensing element consists of two local reflectors inside the actual fiber which can be fabricated with several methods such as dielectric thin-films [143], fiber Bragg gratings [144], or UV-induced mirrors [145]. Alternatively, an EFPI sensor uses an air cavity between two cleaved fiber ends inserted into an alignment ferrule and bonded by laser welding or epoxy adhesive $[136,137]$. The FP cavity can also be fabricated by splicing silica capillary tubing (or hollow core fiber) in between two sections of fiber $[138,141,146]$. This hybrid sensor combines the advantages of intrinsic and extrinsic FP sensors. This sensor differs from an EFPI sensor in that the outer diameter is equal to that of the lead fibers which reduces the size and 
provides geometric continuity in sensing applications. The mechanical strength at the reflecting interfaces is also improved compared to conventional thin-film IFPI sensors. A similar approach was achieved by wet etching combined with splicing and cleaving techniques in a miniature low-finesse FP sensor $[135,139]$. The hybrid sensor provides excellent mechanical properties with an all fused-silica structure and high-temperature capability.

An extrinsic FFPI sensor comprises the lead-in fiber and a reflection mirror. A low-finesse FP cavity can be formed directly by the cleaved end faces of the lead-in fiber and a reflection fiber [136]. As shown in Figure 2.1, the lead-in fiber and the reflection fiber can be bonded together by silica capillary tubing. By selecting silica tubing with different diameters, two types of FFPI sensors can be fabricated by laser bonding [136] [Figure 2.1 (a)] or by fusion splicing [138] [Figure 2.1 (b)]. The fusion-spliced sensor is a hybrid sensor which combines the advantages of IFPI and EFPI sensors [Figure 2.1(b)]. The outer diameter of the hybrid sensor is equal to that of the lead fibers which is smaller than the EFPI sensors. The mechanical strength at the reflecting interfaces is also improved compared to a thin-film IFPI sensor. Compared to the laser-bonded sensor, the fabrication process for the fusion-spliced sensor is relatively easy. Furthermore, the reflection fiber can be cleaved or etched to form a diaphragm-based FFPI sensor [141, 146] [Figure 2.2(a)]. 


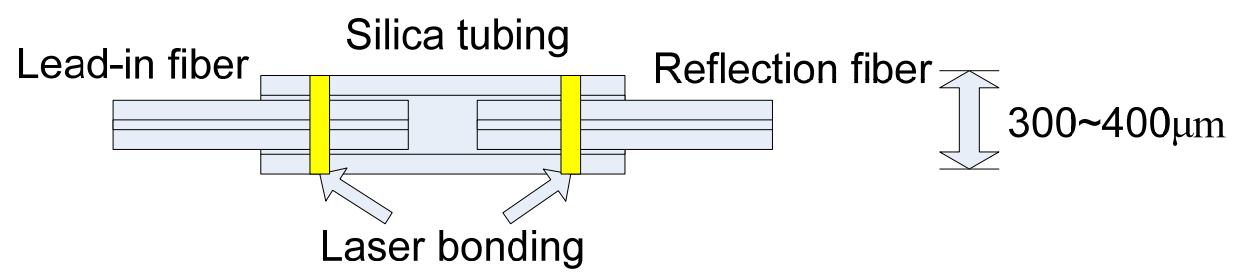

(a)

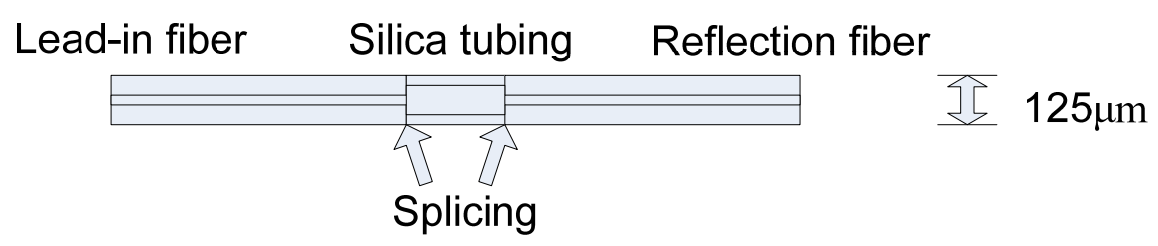

(b)

Figure 2.1 Structure of FFPI sensors. (a) FFPI sensor with silica tubing (ID $>125 \mu \mathrm{m}$ ) fabricated by laser bonding; (b) FFPI sensor with silica tubing $(\mathrm{OD}=125 \mu \mathrm{m})$ fabricated by fusion splicing.

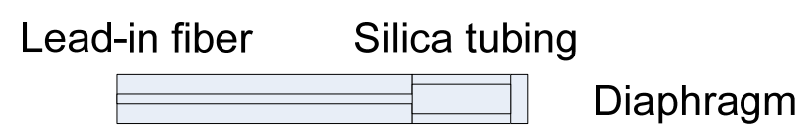

(a)

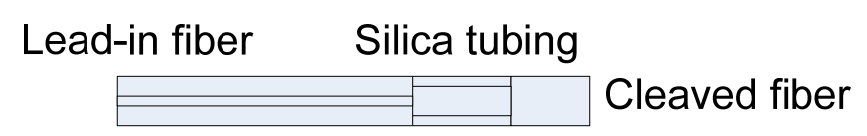

(b)

Figure 2.2 Structure of miniature FFPI sensors. (a) Diaphragm-based FFPI sensor; (b) Multicavity FFPI sensor.

In this chapter, the fabrication of a tubing-based multicavity FP sensor is described. By cleaving the reflection fiber of the hybrid sensor, a second fiber cavity can be formed in addition to the single air-cavity of conventional FP sensors, which is similar to the diaphragm cavity in a pressure FP sensor [135]. Fringe patterns are produced by the interferences between multiple reflection signals at the interfaces of the air and fiber 
cavities. Conventional FP sensors are intended to observe the perturbation of only a single cavity; therefore, the reflections from the interfaces other than the end-faces of the cavity are intentionally minimized to reduce the multiple interferences [133]. Otherwise the reflections must be considered in the analysis [135]. When introducing the additional signal from the outer surface of the reflection fiber, a multicavity FFPI sensor is formed as in Figure 2.2(b). Here the term multicavity refers to the air cavity, fiber cavity and their combination. Each cavity length of the multicavity sensor can be measured with high resolution by the demodulation of the reflection spectrum. Any variations to the end surface of the fiber cavity will change the reflection and thus shift the fringe pattern. Consequently, the self-assembly of polymers can be measured by the multicavity sensor. Further immobilization of proteins onto the sensor will demonstrate its feasibility in biological applications.

\subsection{Sensor fabrication}

The key point in the fabrication of the tubing-based MFPI sensor is fusion splicing the silica capillary tubing with the lead-in fiber and the reflection fiber. The essential procedures are illustrated in Figure 2.3. 


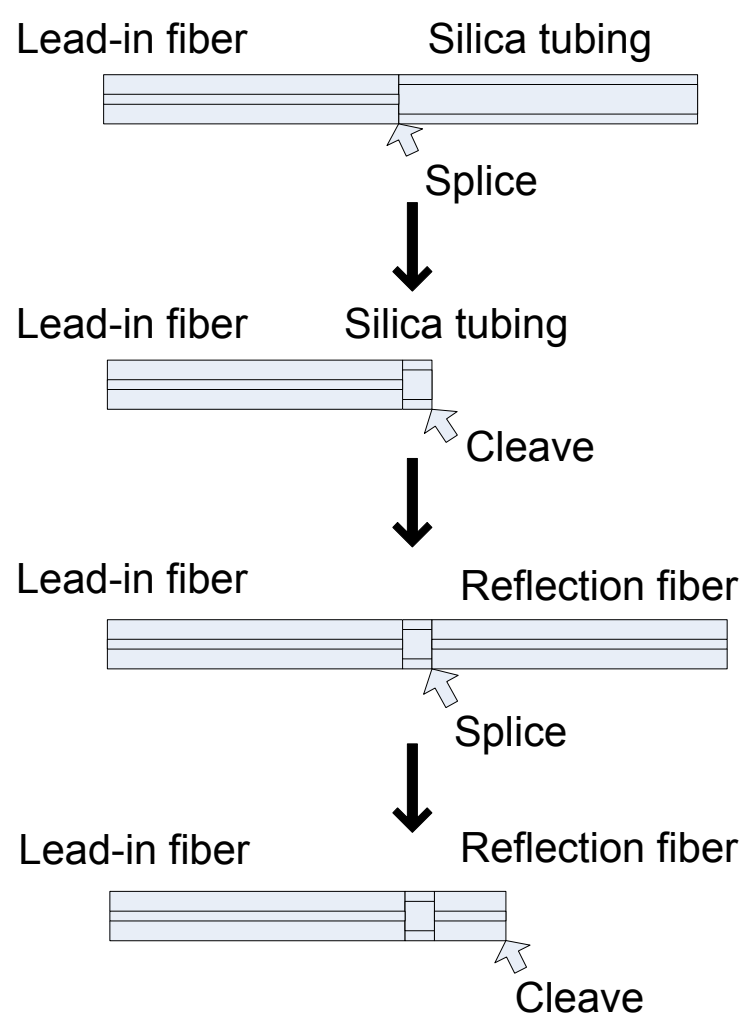

Figure 2.3 Procedures in fabrication of multicavity FFPI sensor.

A silica capillary tube [50- $\mu \mathrm{m}$ inside diameter (ID) and 126- $\mu \mathrm{m}$ outside diameter (OD), Polymicro] was first spliced onto a single mode fiber (Corning SMF-28). By cleaving and splicing with another single mode fiber, a Fabry-Perot cavity was formed by the reflections from the two fiber-air interfaces. Air cavity sizes as small as $10 \mu \mathrm{m}$ can be achieved by cleaving the tubing under a microscope. Another fiber cavity was then created by cleaving the reflection fiber. The size of the fiber cavity can be as small as 10 $\mu \mathrm{m}$, limited by cleaving, and up to the centimeter range, limited by the coherence length of the light source used to interrogate the sensor. The structure of the multicavity sensor is shown in Figure 2.4. Figure 2.5 is a picture of a multicavity sensor under a microscope (Zeiss Axiovert 25). The lower frequency envelope shown in Figure 2.6 is due to the air cavity. The overall fringe pattern of the sensor is due to the interference between the 
reflection signals of the three fiber-air interfaces. It is possible to demodulate the signal to obtain both cavity lengths with high resolution and high accuracy.

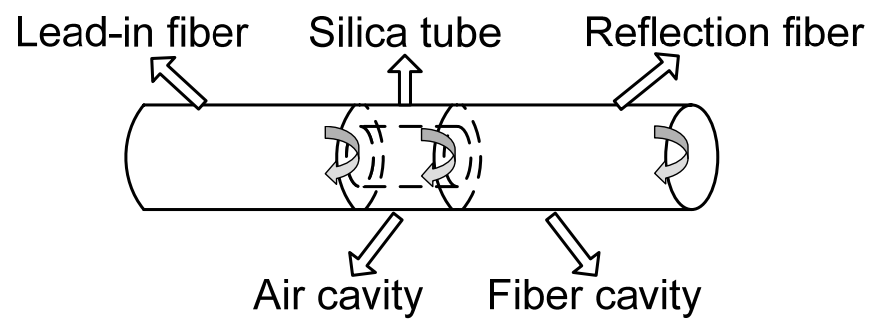

Figure 2.4 Structure of the multicavity FP sensor.

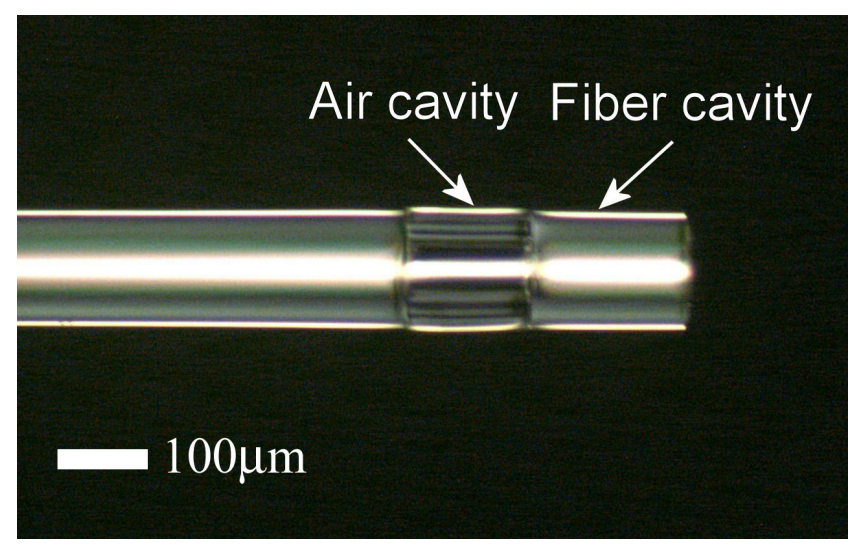

Figure 2.5 Picture of a tubing-based multicavity FP sensor (Zeiss Axiovert 25 microscope). 


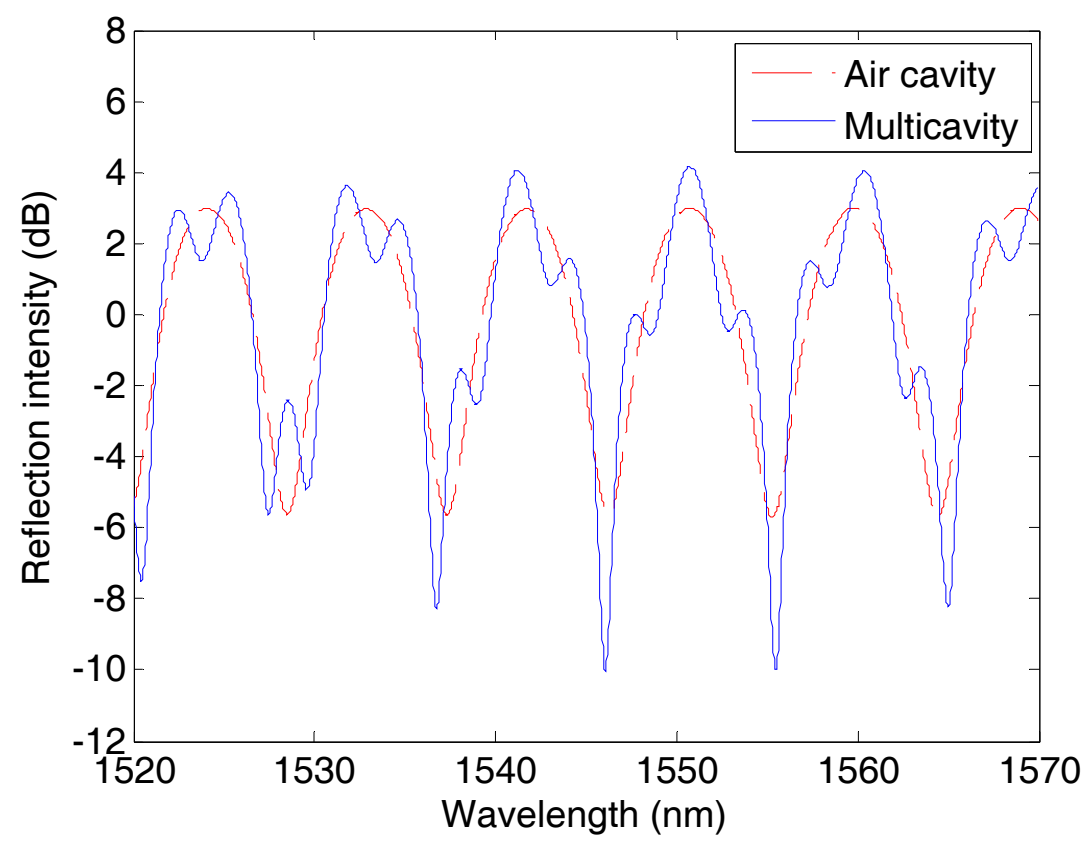

Figure 2.6 Typical reflection spectrum of a multicavity FP sensor.

In order to obtain high visibility and high reflection power, low splicing arc power and short arc time are preferred to reduce the deformation of the interfaces. At the same time, in order to maintain the strength of the splicing point the arc power should not be too low. There is similar requirement on the arc duration. Table 2.1 compares the different arc conditions for multicavity sensor fabrication and normal single mode (SM) fiber splicing (Sumitomo Type-36 Fusion Splicer).

Table 2.1 Comparison of the arc conditions (Sumitomo Type-36 Fusion Splicer) for multicavity sensor fabrication and normal SM fiber splicing.

\begin{tabular}{|l|c|c|}
\hline & Multicavity sensor & Normal SM-SM \\
\hline Arc duration (seconds) & 0.4 & 1.5 \\
\hline Arc power (R.U.) & 5 & 30 \\
\hline
\end{tabular}


The deformation loss can be monitored by a component testing system (CTS, Micron Optics SI720) during splicing. Normally it is lower than $0.5 \mathrm{~dB}$. The reflection signals that couple into the lead-in fiber are affected by the alignment of the lead-in fiber and the reflection fiber. The majority of the loss is due to lateral and angular misalignment. By monitoring the reflection and transmission signal of the FP cavity during fabrication, it is possible to optimize the lateral and angular alignment. The loss from longitudinal misalignment is related to the air cavity size [147] which can be reduced with a small air cavity.

\subsection{Theoretical analysis}

\subsubsection{Conventional analysis for single-cavity Fabry-Perot sensor}

For low-finesse single-cavity FP sensors, it is reasonable to neglect the multiple reflections. A two-beam interferometer model is a suitable simplification

$$
I=I_{1}+I_{2}+2 \sqrt{I_{1} I_{2}} \cos \phi
$$

where $I$ is the light intensity received at the photodetector, $I_{1}$ and $I_{2}$ are the light intensity reflected from each interface, $\phi$ is the total phase shift from the cavity. The total phase shift includes the transmission phase shift, reflection phase shift and coupling phase shift, which can be expressed as [147]

$$
\phi=\frac{4 \pi n d}{\lambda}+\pi+\phi_{0}
$$

where $n$ is the refractive index of the medium in FP cavity, $d$ is the cavity size, $\lambda$ is the wavelength, $\phi_{0}$ is the coupling phase shift from the misalignment. The first item in Eq. 2.2 is the transmission phase shift due to the light propagation in the cavity. For the FFPI sensor in Figure 2.7, there is a $\pi$ phase shift upon reflection at a lower refractive index medium (air) from a higher refractive index medium (fiber). Substituting Eq. 2.2 into Eq. 2.1 yields 


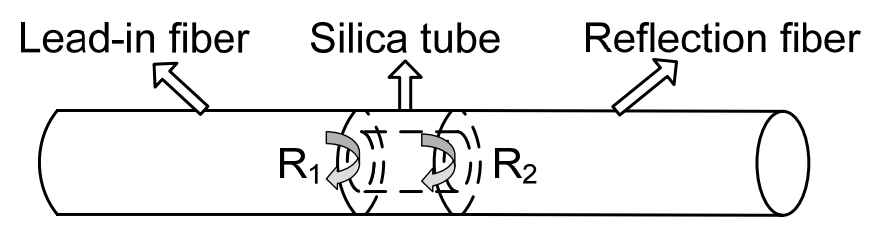

Figure 2.7 Structure of the low-finesse single-cavity FP sensor.

$$
I=I_{1}+I_{2}-2 \sqrt{I_{1} I_{2}} \cos \left(\frac{4 \pi n d}{\lambda}+\phi_{0}\right) .
$$

In order to obtain high resolution and large dynamic range, single peak or valley tracing in conjunction with multiple-peak or multiple-valley demodulation were applied for the white-light interferometric sensor [142]. From Eq. 2.3, the wavelength of a valley point in the reflection spectrum satisfies

$$
\frac{4 \pi n d}{\lambda}+\phi_{0}=2 m \pi
$$

where the order number $m$ is a positive integer. The optical thickness of the cavity is

$$
n d=\frac{\left(2 m \pi-\phi_{0}\right) \lambda}{4 \pi}=\frac{k \lambda}{2}
$$

where $k=m-\phi_{0} /(2 \pi)$ is a constant for a given order number $m$. The resolution in opticalthickness measurement is related to the wavelength detection. The relative error of the single-valley demodulation can be expressed as

$$
\left|\frac{\delta(n d)}{n d}\right|_{s}=\left|\frac{\delta \lambda}{\lambda}\right| \text {. }
$$

Here $\delta \lambda$ is the absolute error of the valley position. Multiple-peak or multiple-valley demodulation can eliminate the ambiguity of the absolute optical thickness. Adjacent valley points in the reflection spectrum satisfy

$$
\begin{aligned}
& n d=\frac{k \lambda_{1}}{2}, \\
& n d=\frac{(k+1) \lambda_{2}}{2},
\end{aligned}
$$


where $\lambda_{1}$ and $\lambda_{2}\left(\lambda_{1}>\lambda_{2}\right)$ are the wavelengths of two adjacent valley points. The optical thickness can be derived as

$$
n d=\frac{\lambda_{1} \lambda_{2}}{2\left(\lambda_{1}-\lambda_{2}\right)} .
$$

The absolute error of multiple-valley demodulation is

$$
\begin{aligned}
\delta(n d) & \approx \sum_{i=1}^{2}\left|\frac{\partial(n d)}{\partial \lambda_{i}}\right| \delta \lambda \\
& =\frac{\lambda_{1}^{2}+\lambda_{2}^{2}}{2\left(\lambda_{1}-\lambda_{2}\right)^{2}} \delta \lambda .
\end{aligned}
$$

The wavelengths $\lambda_{1}$ and $\lambda_{2}$ can be expressed as

$$
\begin{aligned}
& \lambda_{1}=\lambda+\Delta \lambda, \\
& \lambda_{2}=\lambda-\Delta \lambda,
\end{aligned}
$$

where $\Delta \lambda$ is half of the difference between the wavelengths $\lambda_{1}$ and $\lambda_{2}, \lambda$ is the median of $\lambda_{1}$ and $\lambda_{2}(\Delta \lambda<<\lambda)$. Substituting Eq. 2.10 into Eq. 2.9, the relative error of multiple-valley demodulation is

$$
\left|\frac{\delta(n d)}{n d}\right|_{m} \approx\left|\frac{\delta \lambda}{\Delta \lambda}\right| .
$$

It is obvious that the single-valley demodulation has much smaller relative error since

$$
\frac{\left|\frac{\delta(n d)}{n d}\right|_{s}}{\left|\frac{\delta(n d)}{n d}\right|_{m}} \approx \frac{\Delta \lambda}{\lambda}<<1 .
$$

For a single-cavity FP sensor with an air gap from $10 \mu \mathrm{m}$ to $100 \mu \mathrm{m}$, the relative error of multiple-valley demodulation is about 25.8 to 258 times of that from singlevalley demodulation ( $\lambda=1550 \mathrm{~nm}$ ). However, single-valley demodulation can not eliminate the ambiguity in optical-thickness measurement due to unknown order number $m$ and constant $k$. This difficulty can be overcome by first estimating the order number $m$ or constant $k$ from multiple-valley demodulation and then calculating the optical 
thickness by single-valley demodulation [142]. The rough $k$ value can be calculated from Eq. 2.7

$$
k=\frac{\lambda_{2}}{\lambda_{1}-\lambda_{2}} .
$$

According to Eq. 2.5, the order number $m$ can be estimated as

$$
m=k+\frac{\phi_{0}}{2 \pi} .
$$

The order number should be an integer, so the accurate value is found by rounding towards the nearest integer

$$
m=\left[k+\frac{\phi_{0}}{2 \pi}\right],
$$

where the square brackets mean to round up. Here the coupling phase shift $\phi_{0}$ can be calculated from the estimated cavity length, which is similar to the analysis of spice loss of two single-mode fibers [148]. When the lateral and angular misalignments are minimized during fabrication, the phase shift is mainly due to the longitudinal misalignment:

$$
\phi_{0}=-\operatorname{atan}(G / 2),
$$

where $G=Z /\left(K S^{2}\right)$ is the longitudinal displacement factors. $Z=2 d / a$ is the normalized longitudinal displacement, $d$ is the air cavity length, $a$ is the fiber core radius. The effective displacement is two times the cavity length because the incident light reflects from the reflection fiber and couples back into the lead-in fiber. $K=k a$ is the normalized wave number, $S=s / a=w /(\sqrt{2} a)$ is the normalized intensity-based spot size radius, $w$ is the amplitude-based spot size radius and can be approximated by the empirical formula [149]:

$$
\frac{w}{a}=0.65+\frac{1.619}{V^{3 / 2}}+\frac{2.879}{V^{6}} .
$$

Here $a=4.1 \mu \mathrm{m}, V=2.15, S=0.84$ (Corning SMF-28, $\lambda=1550 \mathrm{~nm}$ ). Figure 2.8 shows the relationship between the coupling phase shift $\phi_{0}$ and the cavity length $d$. Compared to the transmission phase shift in Eq. 2.3, the coupling phase shift changes much more slowly 
with the cavity length. If the measurement is in a narrow range of cavity lengths [135, 150], the coupling shift can be estimated as a constant value. Therefore the change in total phase shift is directly proportional to variations of the cavity length.

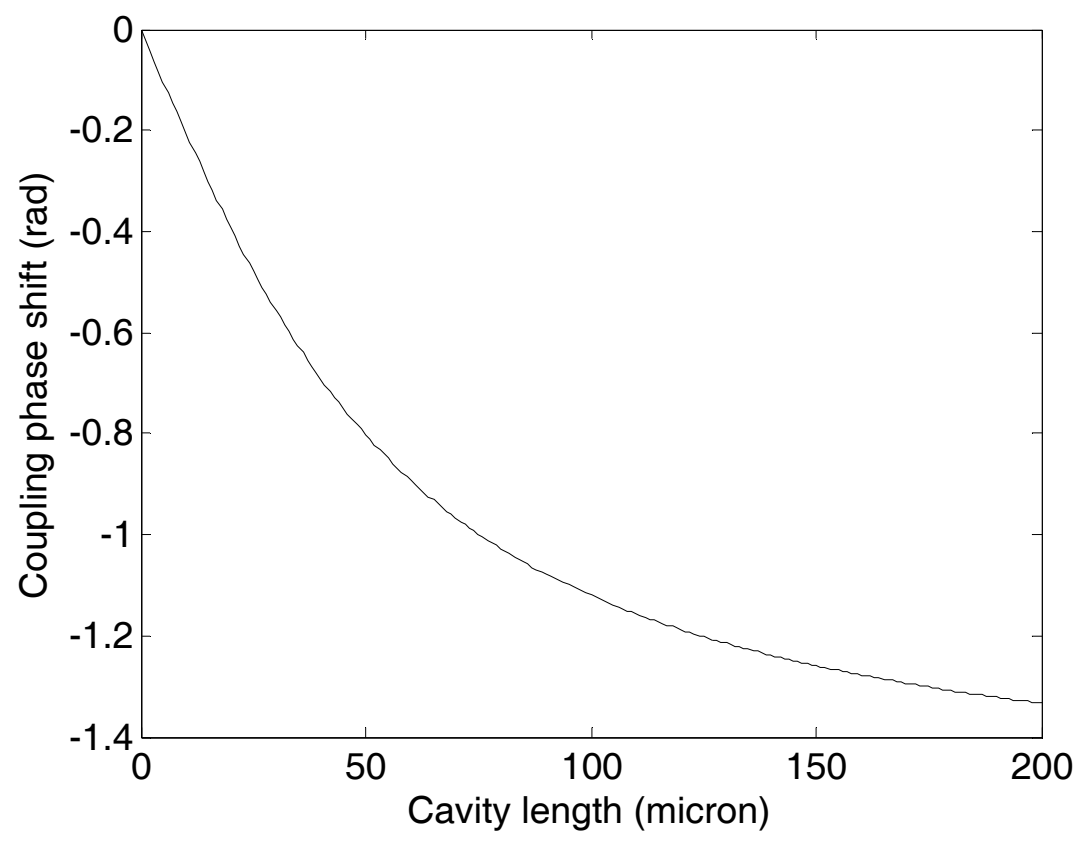

Figure 2.8 Coupling phase shift verses the cavity length.

In low-finesse FP sensors [135, 142, 150], the incident light is transmitted through an air gap, reflected by another fiber or diaphragm, and coupled back into the lead-in fiber. The fringe visibility in the reflection spectrum is closely related to the power coupling efficiency. If there is no misalignment in lateral and angular direction between the lead-in fiber and reflection fiber, the coupling efficiency can also be expressed as a function of the longitudinal displacements [148]:

$$
T=\frac{4}{G^{2}+4} .
$$

Figure 2.9 shows the power coupling coefficient verses the cavity length. The coupling efficiency, as well as the fringe visibility, decreases rapidly with an increase in cavity length. Short air gaps (less than $100 \mu \mathrm{m}$ ) are preferred in single-cavity FP sensor for better fringe visibility $[135,150]$. 


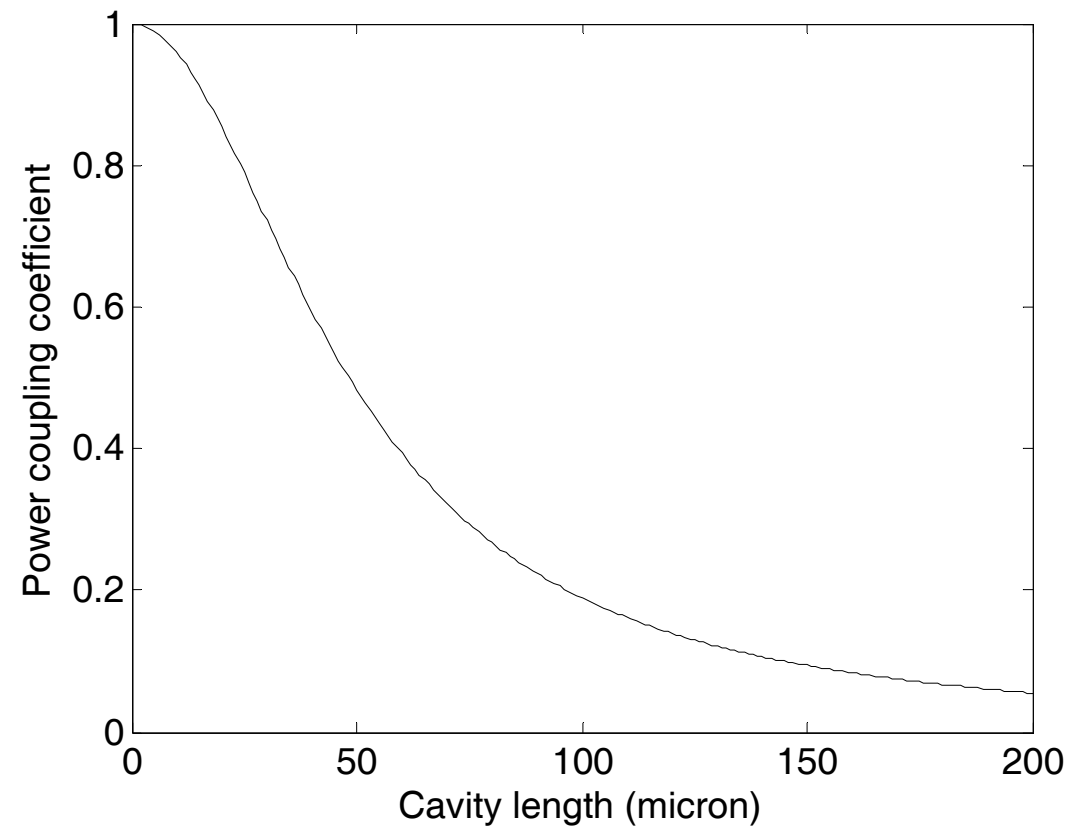

Figure 2.9 Power coupling coefficient verses the cavity length.

\subsubsection{Matrix optics for FP sensor}

Multiple-beam interference can be evaluated by matrix formalism [151]. The multiple reflections neglected in section 2.3.1 are considered here. The multilayer configuration is shown in Figure 2.10. Light is incident on the system from left to right. In any layer $\mathrm{j}, E_{r j}$ is the field component that is traveling to the right; $E_{l j}$ is traveling to the left. The fields on the left side of a layer are represented by unprimed notation and the fields on the right by primed notation. The field components on the left side of layer $\mathrm{j}$ can be expressed as column vector $E_{j}=\left(\begin{array}{c}E_{l j} \\ E_{r j}\end{array}\right)$ and $E_{j}{ }^{\prime}=\left(\begin{array}{c}E_{l j}{ }^{\prime} \\ E_{r j}{ }^{\prime}\end{array}\right)$ represents the fields on the right side of layer $\mathrm{j}$. The field transition between interface $\mathrm{i}-\mathrm{j}$ can be expressed as

$$
E_{i}{ }^{\prime}=H_{i j} E_{j},
$$

where $H_{i j}$ is the interface transition matrix 


$$
H_{i j}=\frac{1}{\tau_{i j}}\left(\begin{array}{cc}
1 & \rho_{i j} \\
\rho_{i j} & 1
\end{array}\right) .
$$

$\rho_{i j}$ and $\tau_{i j}$ are the reflection coefficient and transmission coefficient of interface $\mathrm{i}-\mathrm{j}$

respectively. For the field propagation in layer $\mathrm{j}$

$$
E_{j}=L_{j} E_{j}{ }^{\prime} .
$$

$L_{j}$ is the layer propagation matrix defined as

$$
L_{j}=\left(\begin{array}{cc}
\exp \left(-i \beta_{j}\right) & 0 \\
0 & \exp \left(i \beta_{j}\right)
\end{array}\right),
$$

where $\beta_{j}=\frac{2 \pi}{\lambda_{0}} n_{j} d_{j}$ for normal incidence. So

$$
\begin{aligned}
E_{i}{ }^{\prime} & =H_{i j} E_{j} \\
& =H_{i j} L_{j} E_{j}{ }^{\prime} \\
& =H_{i j} L_{j} H_{j k} E_{k},
\end{aligned}
$$

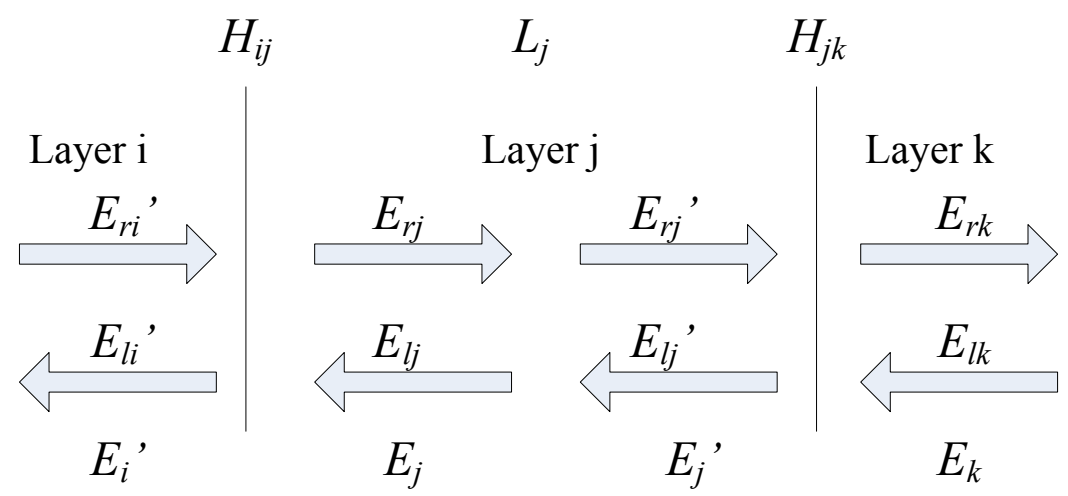

Figure 2.10 Notation for waves traveling over the interface and propagation inside the layer.

In a similar manner we can obtain the relationships between the fields in the incident medium and the final medium $(\mathrm{N})$

$$
E_{1}{ }^{\prime}=S_{1 N} E_{N} .
$$

$S_{1 N}$ is the stack matrix that represents the effects of all the layers with multiple reflections 


$$
S_{1 N}=H_{12} L_{2} \ldots L_{N-1} H_{N-1, N} .
$$

$S_{1 N}$ can be calculated if $\rho_{i j}, \tau_{i j}$ and $\beta_{j}$ are known for each layer. Then it is easy to find the relationship between the fields on either side of the multilayer system

$$
\begin{aligned}
\left(\begin{array}{c}
E_{l 1}{ }^{\prime} \\
E_{r 1}{ }^{\prime}
\end{array}\right) & =\left(\begin{array}{ll}
S_{11} & S_{12} \\
S_{21} & S_{22}
\end{array}\right)\left(\begin{array}{c}
0 \\
E_{r N}
\end{array}\right) \\
& =\left(\begin{array}{l}
S_{12} E_{r N} \\
S_{22} E_{r N}
\end{array}\right) .
\end{aligned}
$$

Therefore the reflectance $\mathrm{R}$ and transmittance $\mathrm{T}$ are

$$
\begin{gathered}
R=|\rho|^{2}=\left|\frac{S_{12}}{S_{22}}\right|^{2}, \\
T=\frac{n_{N}}{n_{1}}|\tau|^{2}=\frac{n_{N}}{n_{1}}\left|\frac{1}{S_{22}}\right|^{2} .
\end{gathered}
$$

\subsubsection{Single cavity sensor}

For the single cavity sensor in Figure 2.7, an air gap of index $n_{2}$ is between the fibers of index $n_{1} . n_{1}=1.45, n_{2}=1$. The stack matrix can be expressed as

$$
S=H_{12} L_{2} H_{21} .
$$

The interface transition matrices are

$$
H_{12}=\frac{1}{\tau_{12}}\left(\begin{array}{cc}
1 & \rho_{12} \\
\rho_{12} & 1
\end{array}\right), H_{21}=\frac{1}{\tau_{21}}\left(\begin{array}{cc}
1 & \rho_{21} \\
\rho_{21} & 1
\end{array}\right) .
$$

The layer propagation matrix is

$$
L_{2}=\left(\begin{array}{cc}
\exp (-i \beta) & 0 \\
0 & \exp (i \beta)
\end{array}\right)
$$

where $\beta=\frac{2 \pi n_{2} d}{\lambda_{0}}+\phi_{0}{ }^{\prime}$ is the phase shift of the air cavity. $\phi_{0}{ }^{\prime}$ is the coupling phase shift from the misalignment.

Substituting Eq. 2.30, 2.31 into Eq. 2.29 yields 


$$
S=\frac{1}{\tau_{12} \tau_{21}}\left(\begin{array}{cc}
\exp (-i \beta)+\rho_{12} \rho_{21} \exp (i \beta) & \rho_{21} \exp (-i \beta)+\rho_{12} \exp (i \beta) \\
\rho_{12} \exp (-i \beta)+\rho_{21} \exp (i \beta) & \rho_{12} \rho_{21} \exp (-i \beta)+\exp (i \beta)
\end{array}\right) .
$$

The reflectance and transmittance are

$$
\begin{aligned}
& R=\frac{\rho_{12}{ }^{2}+\rho_{21}{ }^{2}+2 \rho_{12} \rho_{21} \cos 2 \beta}{1+\rho_{12}{ }^{2} \rho_{21}{ }^{2}+2 \rho_{12} \rho_{21} \cos 2 \beta}, \\
& T=\frac{\tau_{12}{ }^{2} \tau_{21}{ }^{2}}{1+\rho_{12}{ }^{2} \rho_{21}{ }^{2}+2 \rho_{12} \rho_{21} \cos 2 \beta},
\end{aligned}
$$

where $\rho_{12}$ and $\rho_{21}$ are reflection coefficients. For the single cavity configuration in Figure 2.1,

$$
\rho_{12}=-\rho_{21}=\frac{n_{1}-n_{2}}{n_{1}+n_{2}}>0 .
$$

$R_{1}=\rho_{12}{ }^{2}, R_{2}=\left|\rho_{21}\right|^{2}, T_{1}=\tau_{12} \tau_{21}$ are the intensity reflection coefficient and transmission coefficient of interface 1-2. The reflectance and transmittance become

$$
\begin{aligned}
& R=\frac{R_{1}+R_{2}-2 \sqrt{R_{1} R_{2}} \cos 2 \beta}{1+R_{1} R_{2}-2 \sqrt{R_{1} R_{2}} \cos 2 \beta}, \\
& T=\frac{T_{1}^{2}}{1+R_{1} R_{2}-2 \sqrt{R_{1} R_{2}} \cos 2 \beta} .
\end{aligned}
$$

When reflection from the interface is low $\left(R_{1}, R_{2}<<1\right)$, multiple reflections can be neglected. The reflection can be approximated as

$$
\begin{aligned}
R & \approx R_{1}+R_{2}-2 \sqrt{R_{1} R_{2}} \cos 2 \beta \\
& =R_{1}+R_{2}-2 \sqrt{R_{1} R_{2}} \cos \left(\frac{4 \pi n_{2} d}{\lambda_{0}}+2 \phi_{0}{ }^{\prime}\right),
\end{aligned}
$$

which is similar to Eq. 2.3 since the two-beam interferometric model is the simplified model without considering the multiple reflections. The comparison between the matrix optics model and two-beam model of the single-cavity sensor reflection spectrum is shown in Figure 2.11. When considering multiple reflections, the peak reflection intensity is relatively lower. The differences in the valley reflection intensities are much smaller than the peaks, while the wavelength positions of peaks and valleys are the same. To 
verify the equalization of the peak and valley wavelength positions, we can find the extrema of Eq. 2.36 and Eq. 2.38 by differentiation. Eq. 2.36 and Eq. 2.38 can be expressed as

$$
R=\frac{A+f(\lambda)}{B+f(\lambda)}
$$

and

$$
R^{\prime}=A+f(\lambda)
$$

respectively where $A=R_{1}+R_{2}, B=1+R_{1} R_{2}, f(\lambda)=-2 \sqrt{R_{1} R_{2}} \cos 2 \beta$. Therefore,

$$
\begin{gathered}
\frac{d R}{d \lambda}=\frac{(B-A)}{(B+f)^{2}} \frac{d f}{d \lambda}=0 \Rightarrow \frac{d f}{d \lambda}=0, \\
\frac{d R^{\prime}}{d \lambda}=0 \Rightarrow \frac{d f}{d \lambda}=0,
\end{gathered}
$$

since

$$
B-A=1+R_{1} R_{2}-R_{1}-R_{2}>0
$$

and

$$
B+f=1+R_{1} R_{2}-2 \sqrt{R_{1} R_{2}} \cos 2 \beta>0 .
$$

So the two models have the same peak and valley wavelength positions in the reflection spectrum. In data processing of the white-light interferometric sensor, peak or valley tracing methods are usually applied [142]. Here we find that the two-beam model for single cavity FP sensors is still valid since the peak or valley positions are not affected by ignoring the multiple reflections. The simplification itself will not cause any systematic errors. However we will mention in a later section that the tracing method may not be directly applied to the multicavity sensor because of the multiple interferences involved. Therefore we will apply a curve-fitting method instead in the analysis of the multicavity situation. 


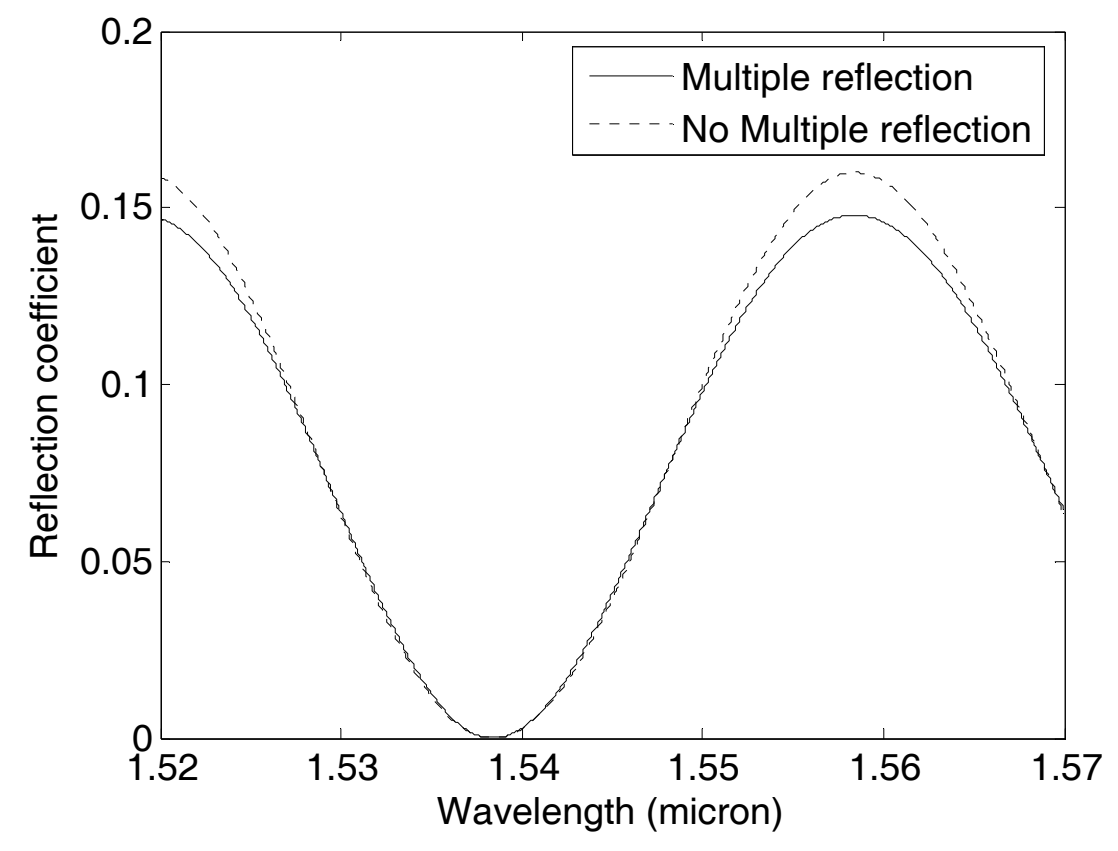

Figure 2.11 Comparison of the theoretical models for single FP cavity sensor. Here assume $\mathrm{R} 1=0.04, \mathrm{R} 2=0.04, \mathrm{~d}=50 \mu \mathrm{m}$ and $\phi_{0}{ }^{\prime}=0$.

\subsubsection{Multicavity sensor}

For the layer structure shown in Figure 2.4, the composite cavity matrix can be expressed as

$$
S=H_{12} L_{2} H_{23} L_{3} H_{34} .
$$

From Eq. 2.20 the interface transition matrices are

$$
H_{12}=\frac{1}{\tau_{12}}\left(\begin{array}{cc}
1 & \rho_{12} \\
\rho_{12} & 1
\end{array}\right), H_{23}=\frac{1}{\tau_{23}}\left(\begin{array}{cc}
1 & \rho_{23} \\
\rho_{23} & 1
\end{array}\right), H_{34}=\frac{1}{\tau_{34}}\left(\begin{array}{cc}
1 & \rho_{34} \\
\rho_{34} & 1
\end{array}\right),
$$

and from Eq. 2.22 the layer propagation matrices are

$$
L_{2}=\left(\begin{array}{cc}
\exp \left(-i \beta_{2}\right) & 0 \\
0 & \exp \left(i \beta_{2}\right)
\end{array}\right), L_{3}=\left(\begin{array}{cc}
\exp \left(-i \beta_{3}\right) & 0 \\
0 & \exp \left(i \beta_{3}\right)
\end{array}\right)
$$


where $\beta_{2}=\frac{2 \pi n_{2} d_{2}}{\lambda}+\phi_{0}{ }^{\prime}$ is the phase shift of the air cavity, $\beta_{3}=\frac{2 \pi n_{1} d_{3}}{\lambda}$ is the phase shift of the fiber cavity. $n_{1}=1.45, n_{2}=1$ are the refractive indices of fiber and air. $\phi_{0}{ }^{\prime}$ is the coupling phase shift from the longitudinal misalignment. The intensity reflection coefficient of the multicavity sensor is

$$
\begin{aligned}
& R=\frac{\rho_{12}{ }^{2}+\rho_{23}{ }^{2}+\rho_{34}{ }^{2}+\rho_{12}{ }^{2} \rho_{23}{ }^{2} \rho_{34}{ }^{2}+2 \rho_{12} \rho_{23}\left(\rho_{34}{ }^{2}+1\right) \cos \left(2 \beta_{2}\right)+}{1+\rho_{12}{ }^{2} \rho_{23}{ }^{2}+\rho_{23}{ }^{2} \rho_{34}{ }^{2}+\rho_{12}{ }^{2} \rho_{34}{ }^{2}+2 \rho_{12} \rho_{23}\left(\rho_{34}{ }^{2}+1\right) \cos \left(2 \beta_{2}\right)+} \\
& \frac{2 \rho_{23} \rho_{34}\left(\rho_{12}{ }^{2}+1\right) \cos \left(2 \beta_{3}\right)+2 \rho_{12} \rho_{34} \cos \left(2 \beta_{2}+2 \beta_{3}\right)+2 \rho_{12} \rho_{23}{ }^{2} \rho_{34} \cos \left(2 \beta_{2}-2 \beta_{3}\right)}{2 \rho_{23} \rho_{34}\left(\rho_{12}{ }^{2}+1\right) \cos \left(2 \beta_{3}\right)+2 \rho_{12} \rho_{34} \cos \left(2 \beta_{2}+2 \beta_{3}\right)+2 \rho_{12} \rho_{23}{ }^{2} \rho_{34} \cos \left(2 \beta_{2}-2 \beta_{3}\right)}
\end{aligned}
$$

where $\rho_{12}, \rho_{23}$ and $\rho_{34}$ are reflection coefficients. For the symmetric configuration in Figure 2.4,

$$
\rho_{12}=-\rho_{23}=\rho_{34}=\frac{n_{1}-n_{2}}{n_{1}+n_{2}}>0 .
$$

The intensity reflection coefficients are $R_{1}=\rho_{12}{ }^{2}, R_{2}=\left|\rho_{23}\right|^{2}, R_{3}=\rho_{34}{ }^{2}$. The reflectance coefficient then becomes

$$
\begin{aligned}
& R=\left|\frac{S_{12}}{S_{22}}\right|^{2}=\frac{R_{1}+R_{2}+R_{3}+R_{1} R_{2} R_{3}-2 \sqrt{R_{1} R_{2}}\left(R_{3}+1\right) \cos \left(2 \beta_{2}\right)-}{1+R_{1} R_{2}+R_{2} R_{3}+R_{1} R_{3}-2 \sqrt{R_{1} R_{2}}\left(R_{3}+1\right) \cos \left(2 \beta_{2}\right)-} \\
& \frac{2 \sqrt{R_{2} R_{3}}\left(R_{1}+1\right) \cos \left(2 \beta_{3}\right)+2 \sqrt{R_{1} R_{3}} \cos 2\left(\beta_{2}+\beta_{3}\right)+2 \sqrt{R_{1} R_{3}} R_{2} \cos 2\left(\beta_{2}-\beta_{3}\right)}{2 \sqrt{R_{2} R_{3}}\left(R_{1}+1\right) \cos \left(2 \beta_{3}\right)+2 \sqrt{R_{1} R_{3}} \cos 2\left(\beta_{2}+\beta_{3}\right)+2 \sqrt{R_{1} R_{3}} R_{2} \cos 2\left(\beta_{2}-\beta_{3}\right)}
\end{aligned}
$$

When considering the coupling loss from misalignment and deformation, the effective reflection coefficients are usually smaller than the theoretical prediction. The effective reflection coefficients and phase shifts can be found by fitting the reflection spectrum to the above model. Then the cavity lengths can be calculated from the phase shifts. Multiple reflections are considered for better fitting of the experimental result.

As mentioned in section 2.3.1, the power coupling loss limits the air cavity length to less than $100 \mu \mathrm{m}$ in low-finesse FP sensors. Transverse and angular misalignment in air-cavity fabrication will further deteriorate the fringe visibility and thus reduce the suitable range of air-cavity size. The light source in fiber optic sensing (LED, SLD) usually has a wavelength range of tens of nanometers. In our test, the tunable laser (SI720, 
Micron Optics) is continuously swept across a $50 \mathrm{~nm}$ spectrum $(1520-1570 \mathrm{~nm})$. From Eq. 2.8 , the optical length of the cavity can be expressed as:

$$
n d=\frac{\lambda \lambda^{\prime}}{2\left(\lambda-\lambda^{\prime}\right)} \approx \frac{\lambda^{2}}{2 \Delta \lambda},
$$

where $\lambda$ and $\lambda^{\prime}\left(\lambda>\lambda^{\prime}\right)$ are the wavelengths of adjacent peaks or valleys in the interference spectrum, $\Delta \lambda=\lambda-\lambda$. The central wavelength in the detection range is around $1550 \mathrm{~nm}$. So the fringe period of a $100 \mu \mathrm{m}$ air cavity is

$$
\Delta \lambda \approx \frac{\lambda^{2}}{2 n d}=12 \mathrm{~nm} .
$$

The reflection spectrum of a $100 \mu \mathrm{m}$ cavity has only 4 cycles in $50 \mathrm{~nm}$ wavelength range. It is difficult to demodulate the cavity length in a multicavity sensor by filtering in the wavenumber domain. Therefore we applied a curve fitting method in the demodulation of tubing-based multicavity signals. Figure 2.12 gives a sample spectrum of a multicavity sensor with air cavity size of $32.97 \mu \mathrm{m}$ and fiber cavity size of $113.92 \mu \mathrm{m}$. The lower frequency envelope is mostly due to the air cavity and the higher frequency signal is due to the combined signal of the air and fiber cavities. The peak or valley tracing method cannot be directly applied because of the multiple interferences. The shifts of peaks or valleys are not in linear response to changes of either cavity length. The theoretical fitting by the matrix optics model (Eq. 2.50) is also presented in Figure 2.12. Both of the cavity lengths can be demodulated with $0.1 \mathrm{~nm}$ resolution. 


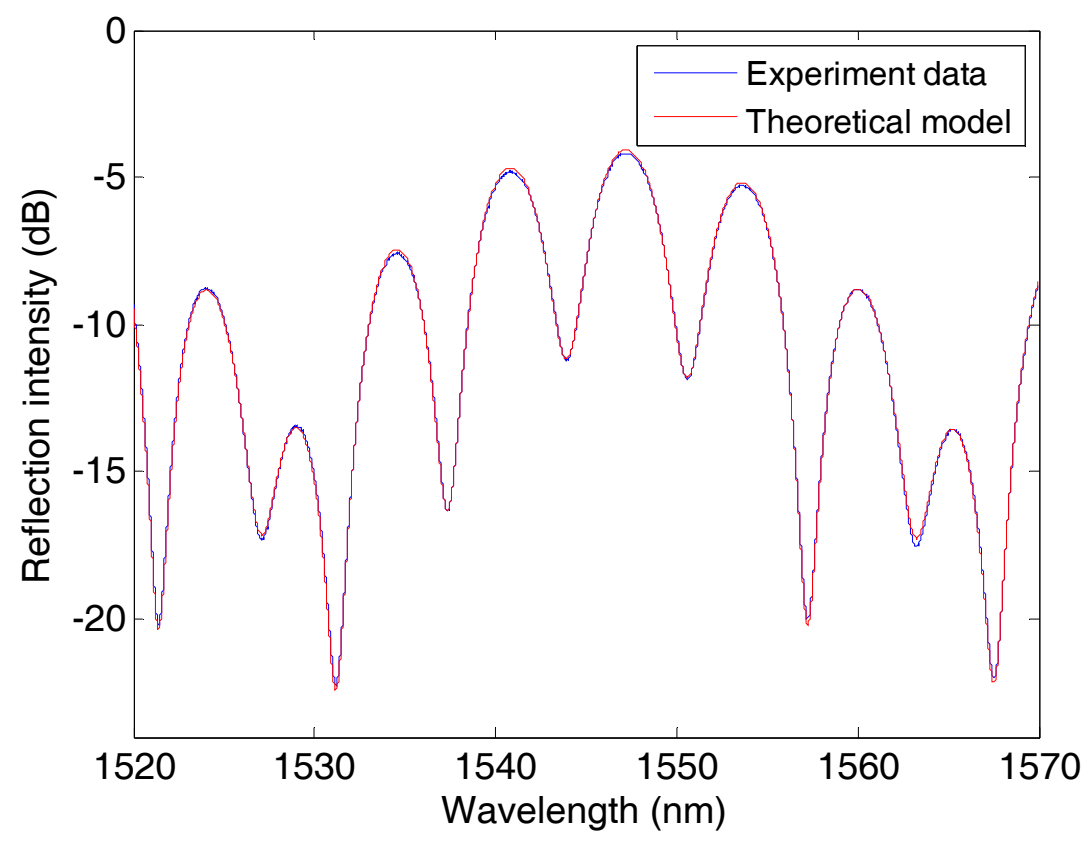

Figure 2.12 Comparison of the experimental data and theoretical fitting for a multicavity FP sensor.

\subsection{Temperature compensation}

The tubing-based multicavity Fabry-Perot interferometric (MFPI) sensor is designed for self-assembled thin-film applications [35]. The MFPI sensor is distinguished by its simplicity, high sensitivity, low cost and capability in the measurement of ultra-thin films in chemical or biological applications by monitoring the changes in the optical thickness of the cavity. However, the changes in optical cavity length induced by the thermo-optic effect and thermal expansion have significant influence on the measurement accuracy. As shown in Figure 2.13, the reflection spectrum of the multicavity sensor results from the multiple interferences between the reflection signals, which is sensitive to the temperature. It is necessary to introduce temperature compensation in highaccuracy measurements at varying temperatures. Temperature compensation in fiber optic sensors can be achieved by multiplexing a separate temperature sensor [152] or creating a compound interferometric cavity from fibers with opposite temperature 
characteristics [153]. Here we report an MFPI sensor with integrated compensation by extracting the temperature information directly from the multicavity structure [154]. The built-in temperature compensation scheme results in advantages such as simple but robust structure, miniature size and resistance to harsh environments.

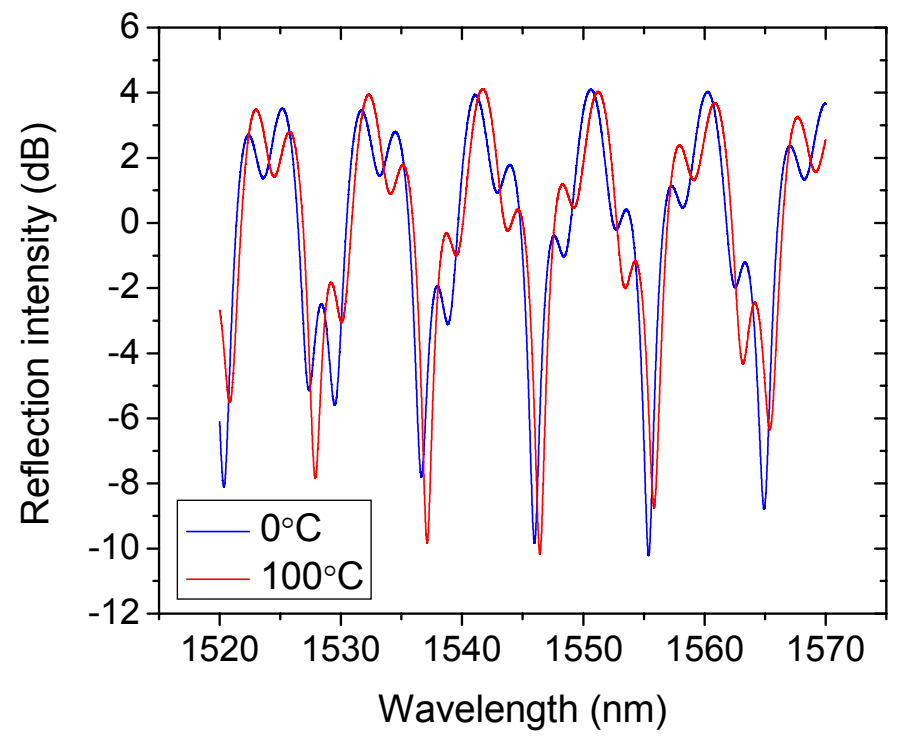

Figure 2.13 Reflection spectra of a multicavity $\mathrm{FP}$ sensor at $0^{\circ} \mathrm{C}$ and $100^{\circ} \mathrm{C}$.

The thin-film sensing is based on the measurement of optical thickness changes of the fiber-film cavity caused by the adsorption of target molecules. The adsorbed thin film can be estimated as an extension of the fiber cavity when the refractive index of the film is similar to the fiber. High sensitivity can be achieved due to the intrinsic nature of Fabry-Perot interferometry. However, the optical thickness of the fiber cavity is also highly dependent on the environment temperature due to the thermo-optic effect and thermal expansion. The change in the optical thickness of the fiber cavity with temperature can be expressed as [155]:

$$
\begin{aligned}
\Delta(n d)_{f} & =\Delta n_{f} \cdot d_{f}+\Delta d_{f} \cdot n_{f} \\
& =\alpha_{n} \Delta T \cdot d_{f}+\alpha_{d} d_{f} \Delta T \cdot n_{f} \\
& =\alpha_{f} n_{f} d_{f} \Delta T,
\end{aligned}
$$


where $\alpha_{n}$ is the thermo-optic coefficient, $\alpha_{d}$ is the thermal expansion coefficient, $\alpha_{f}=\alpha_{n} / n_{f}+\alpha_{d}$ is the effective temperature coefficient. For fused silica $\alpha_{n}=1.0 \times 10^{-5} /{ }^{\circ} \mathrm{C}$, $\alpha_{d}=5.5 \times 10^{-7} /{ }^{\circ} \mathrm{C}, \alpha_{f}=7.4 \times 10^{-6} /{ }^{\circ} \mathrm{C}\left(n_{f}=1.457, \lambda=0.633 \mu \mathrm{m}\right)$ [156]. The thermo-optic effect is the dominant noise factor in the optical thickness measurement. It is more than ten times larger than the effect of the thermal expansion. Therefore the nonlinearity of the thermal expansion effect can be neglected in Eq. 2.53. From the above analysis, any variation of the environmental temperature will affect the accuracy of the thin-film measurement. In particular when the fiber cavity is longer, the absolute change in optical thickness will increase consequently. For an MFPI sensor with a $100 \mu \mathrm{m}$ fiber cavity, the variation in optical thickness over a $100^{\circ} \mathrm{C}$ temperature range for an uncompensated sensor is around $100 \mathrm{~nm}$, which is too large for thin-film measurement. In order to minimize the temperature effect, a shorter fiber cavity is preferred, but this will cause difficulties in the fabrication and signal analysis.

The length of the air cavity also changes with the temperature due to the thermal expansion of the silica tube. Therefore it is possible to compensate the temperature dependence of the fiber cavity by extracting the temperature information from the air cavity. The change in thickness of the air cavity with temperature can be estimated as [157]

$$
\Delta d_{a}=\left(\alpha_{d} \Delta T+\beta_{d} \Delta T^{2}\right) d_{a},
$$

where $\alpha_{d}$ and $\beta_{d}$ are the thermal expansion coefficients of the silica tube, $d_{a}$ is the thickness of the air cavity. Here the nonlinearity of the thermal expansion effect is considered for better fitting. As mentioned in section 2.1, the tubing-based multicavity sensor is based on silica capillary tubing sandwiched between fibers. The size of the air cavity depends on the cleaving and splicing. It is possible to achieve an air cavity size of as small as $10 \mu \mathrm{m}$ when cleaving the tubing under microscope. Another restriction originates from the alignment during splicing. When the cavity size is smaller than $20 \mu \mathrm{m}$, the alignment of air-cavity end-faces becomes worse after splicing. For better visibility of the reflection spectrum, a minimum cavity size of $20 \mu \mathrm{m}$ is required. As in Eq. 2.54 the temperature sensitivity is related to the thermal expansion coefficients and the thickness 
of the air cavity. Due to the low thermal expansion coefficients of silica, a longer air cavity is preferred for temperature measurement. However, as explained in section 2.3.1, the coupling loss from a long air cavity will deteriorate the visibility of the interference fringes and further lower the sensitivity in temperature measurement. Preliminary results show that an air-cavity thickness around $100 \mu \mathrm{m}$ is a reasonable trade-off with good temperature sensitivity and low coupling loss. The optical thickness of the fiber cavity is selected to be longer than the air cavity and able to be differentiated from the air cavity in the wavenumber domain of the reflection spectrum. Here the air-cavity thickness was 133 $\mu \mathrm{m}$ and the optical thickness of the fiber cavity was $242 \mu \mathrm{m}$.

The experimental setup of the MFPI sensor system is shown in Figure 2.14. The reflection spectrum of the sensor was monitored by a component testing system (CTS Micron Optics) and analyzed by a personal computer. The tunable laser of the CTS was coupled into the sensor and the reflection signal was routed back to the CTS detector through the same coupler. The CTS offers a measurement range of 1520-1570 nm with $1 \mathrm{pm}$ accuracy.

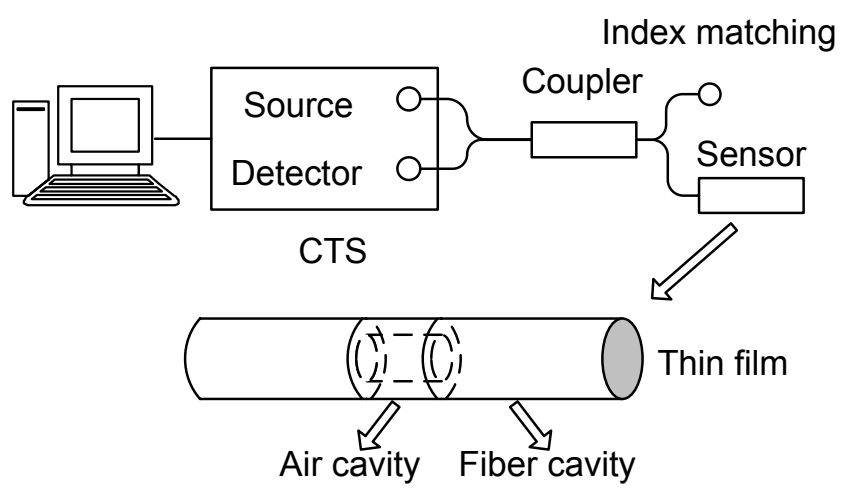

Figure 2.14 Schematic of multicavity FP sensor system for thin-film applications.

Multiple-beam interference from the multicavity FP sensor can be evaluated by matrix formalism (Section 2.3.2). By fitting the spectrum with the theoretical model in Eq. 2.50 , the cavity length can be demodulated with up to $0.1 \mathrm{~nm}$ resolution, limited by the $\mathrm{CTS}$, which corresponds to $2^{\circ} \mathrm{C}$ temperature resolution. The temperature sensitivity is 
limited by the low thermal expansion coefficient of the silica tubing. Higher temperature resolution can be achieved by replacing the silica capillary tubing with other materials or sensors with higher temperature coefficients.

The sensor was first calibrated in an environmental chamber (1020C TestEquity). Figure 2.15 and Figure 2.16 show the changes in air-cavity thickness and fiber-cavity optical thickness with temperature, respectively. The nonlinearity of thermal expansion described in Eq. 2.54 is obvious from the polynomial fitting in Figure 2.15. The thermal expansion of silica shows a nonlinear increase up to about room temperature, followed by an approximately linear range. The total thermal effect on optical thickness changes on the fiber cavity is almost linear with temperature variations, as shown in Figure 2.16. The effective temperature coefficient was fitted as $6.54 \pm 0.06 \times 10^{-6} /{ }^{\circ} \mathrm{C}(\lambda=1.55 \mu \mathrm{m})$. The difference between the experimental results and previous calculation may be due to the different wavelength range. The thermo-optic coefficient of silica becomes smaller for longer wavelength [156]. Moreover, the fiber material may have slightly different thermal effect because of the doping.

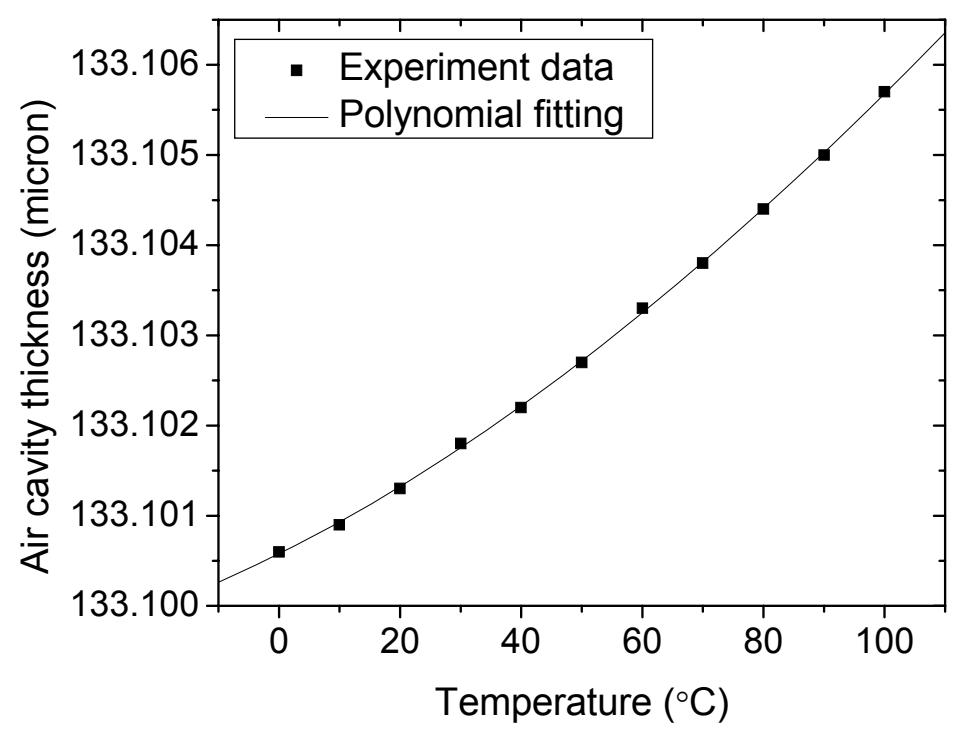

Figure 2.15 Changes in air-cavity thickness with temperature from 0 to $100^{\circ} \mathrm{C}$. 


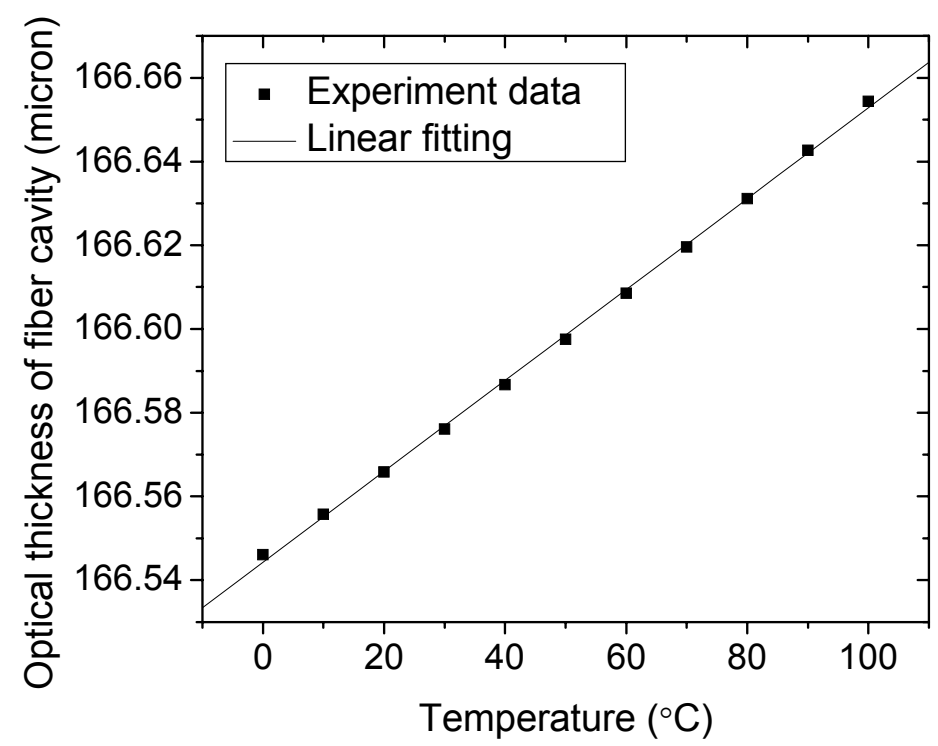

Figure 2.16 Changes in optical thickness of fiber cavity with temperature from 0 to $100^{\circ} \mathrm{C}$.

The relative changes in the air-cavity thickness and the fiber-cavity optical thickness due to temperature variations are shown in Figure 2.17, which can be fit to a polynomial. The change in air-cavity thickness depends on the thermal expansion of the silica tube but is not related to the subsequent adsorption of thin films onto the fiber cavity end. Therefore the thermo-optic and thermal expansion effects of the fiber cavity can be compensated by extracting the temperature information from the air cavity. To demonstrate this, the multicavity sensor was then coated with a polyelectrolyte thin film. By alternately immersing the sensor in polyallylamine hydrochloride (PAH) and polysodium 4-styrenesulfonate (PSS) solutions, a multilayer thin film with structure of $[\mathrm{PAH} / \mathrm{PSS}]_{10}$ was self-assembled onto the sensor end [35]. The film thickness was measured to be $86 \mathrm{~nm}(\mathrm{n}=1.54)$ at room temperature $\left(25^{\circ} \mathrm{C}\right)$ by comparing the optical thickness of the fiber-film cavity with the bare fiber cavity. Since the film is much thinner than the fiber cavity, any variation in the environmental temperature will affect accuracy in the thin-film measurement due to the thermal effects of the fiber cavity. Figure 2.18 
shows the temperature compensation effect on the optical thickness error of the fiber cavity. In the temperature range from 0 to $100^{\circ} \mathrm{C}$, the temperature dependence was about $\pm 76 \mathrm{~nm}$ before compensation. The errors are mostly due to the thermal effects of the fiber cavity itself, which makes the measurement of ultra-thin film under temperature variations difficult. The optical thickness changes of the fiber cavity resulting from the temperature variations can be subtracted by introducing the temperature information from the air cavity. As can be seen in Figure 2.18, the temperature dependence of the optical thickness reduced to about $\pm 5 \mathrm{~nm}$ after compensation, which corresponded to thickness errors of about $\pm 3 \mathrm{~nm}(\mathrm{n}=1.54)$.

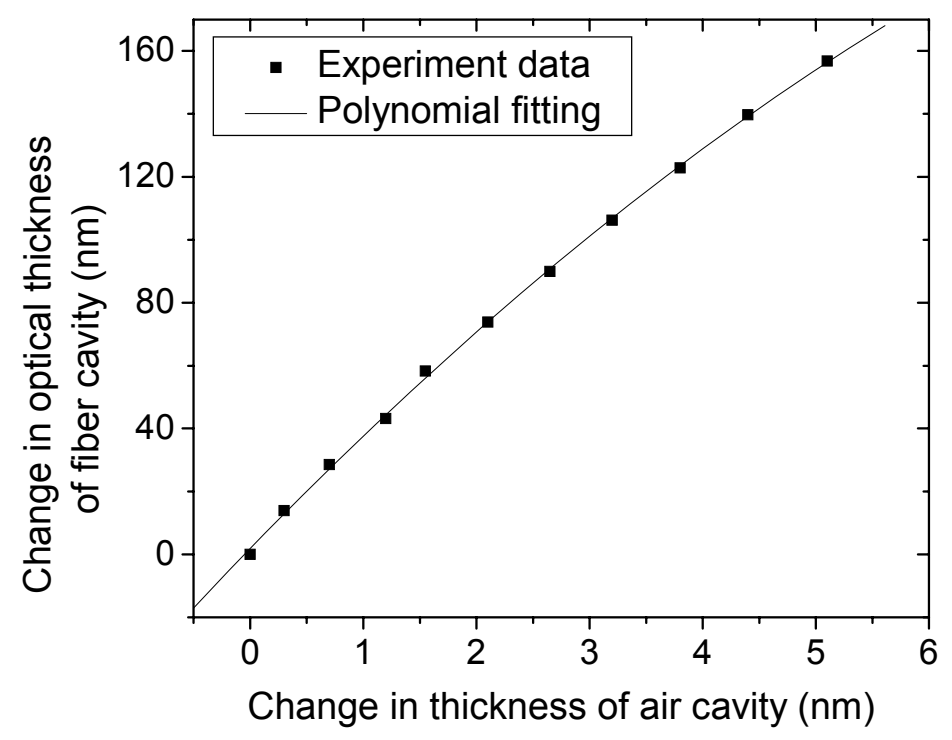

Figure 2.17 Comparison of changes in air-cavity thickness to the fiber-cavity optical thickness of an MFPI sensor with respect to temperature from 0 to $100^{\circ} \mathrm{C}$. 


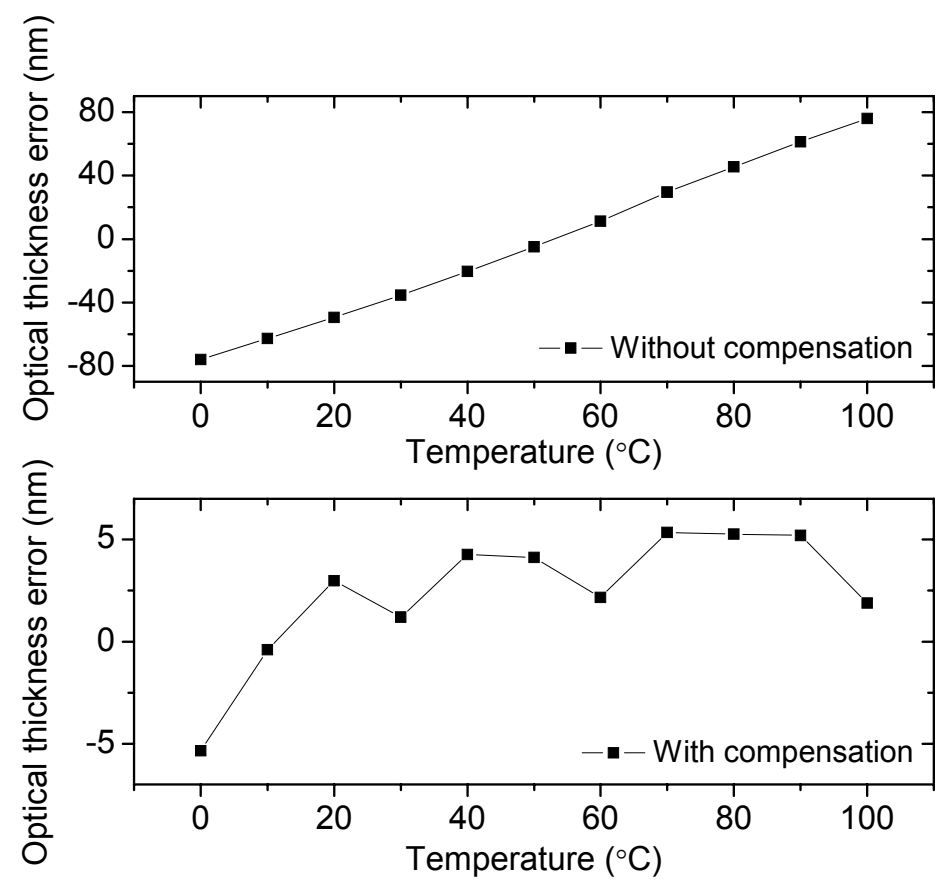

Figure 2.18 Effects of temperature compensation on the optic thickness error of fiber cavity in an MFPI sensor coated with thin film. The upper curve shows the optical thickness error before compensation in the temperature range from 0 to $100^{\circ} \mathrm{C}$. The lower curve shows the error after compensation.

To our knowledge, there is no exact thermal expansion and thermo-optical coefficients for the specific self-assembly in the study. However, we can estimate the effect from similar polymer materials. Generally polymer materials have a positive thermal expansion coefficient and negative thermo-optic coefficient [156]. The thermal expansion coefficient of polystyrene is $6.0 \sim 8.0 \times 10^{-5} /{ }^{\circ} \mathrm{C}$ and the thermo-optical coefficient is $-1.2 \sim-1.4 \times 10^{-4} /{ }^{\circ} \mathrm{C}$. From Eq. 2.53 , the effective temperature coefficient is $-2.75 \sim 0.5 \times 10^{-5} /{ }^{\circ} \mathrm{C}(\mathrm{n}=1.6)$. If the film thickness is $100 \mathrm{~nm}$, the optical thickness change for temperature variation of $100^{\circ} \mathrm{C}$ is $-0.44 \sim 0.08 \mathrm{~nm}$, which is smaller than the compensation error. Therefore the overall variations in the optical thickness of the polymer self-assembly can be neglected compared to the compensation error. Furthermore, the slight variation of the compensated data is most likely caused by 
thickness changes in the thin film because of the release of bound water molecules from the film and reentry from the air due to temperature changes [158].

\subsection{Conclusions}

A tubing-based multicavity fiber-optic FP sensor has been developed for chemical and biological thin-film applications. A fusion-splicing technique is applied in the fabrication of the MFPI sensor. It is easy to control the cavity length during the cleaving and splicing process. The size of the sensor was reduced compared to other extrinsic FP sensors by sandwiching a silica capillary tube between the lead-in fiber and the reflection fiber. An additional fiber cavity was introduced to interferometrically measure the adsorbed thin film. Matrix optics was applied in the analysis of the reflection spectrum from the multicavity sensor. A theoretical model of the multicavity sensor was developed which is in accordance with the experimental results.

The temperature dependence of the fiber-cavity optical thickness can be compensated by extracting the temperature information from the air cavity, greatly reducing the temperature dependence in the thin-film measurement. Thus it provides the possibility to examine the thin-film characteristics under different environment temperatures. The MFPI sensor is applicable not only to the measurement of polyelectrolyte self-assembly but also to the in situ monitoring of the binding process in biosensing applications, including antibody-antigen interactions and DNA hybridization events, as well as gas and chemical sensing. The MFPI sensor may be incorporated into the white light interferometric fiber optic sensor systems developed in our lab. An LED as the low-cost broadband light source and a fiber spectrometer as the detector can provide high stability, high sensitivity, low cost and compact size. 


\section{Chapter 3 Microgap multicavity Fabry-Perot sensor}

\subsection{Introduction}

Among various fiber optic sensors, fiber-optic Fabry-Perot interferometric (FFPI) sensors are distinctive because of the high sensitivity, ease of fabrication, localization and lead insensitivity. As described earlier, FFPI sensors can be classified as extrinsic FabryPerot interferometric (EFPI) sensors and intrinsic Fabry-Perot interferometric (IFPI) sensors. The EFPI sensor is attractive in various applications [134, 136, 137, 140]. However, the EFPI has intrinsic disadvantages such as the difficulty in bonding, nonuniformity in diameter due to the alignment tube and limitation on the cavity size due to coupling loss. The large mismatch in thermal-expansion coefficients will cause severe stress between different materials in sensor construction. The bonding adhesive, such as epoxy, is another potential problem in high temperature environment. The geometric discontinuity will create difficulty in protecting and mounting the sensor in the measurement. The limited cavity length reduces the possible number of multiplexing sensors. In contrast, the IFPI sensor contains the sensing element, i.e. the FP cavity, inside the fiber. The fiber guides light and experiences the perturbation of the interest. IFPI sensors reduce the bonding difficulties experienced in EFPI sensor fabrication, and provide miniature size, continuous geometry, robust structure and versatile installation.

The local reflectors or mirrors inside the fiber can be fabricated with various methods such as dielectric thin-films [133, 143, 159], fiber Bragg gratings [144], and UV-induced mirrors [145]. Single-layer [133, 143] or multilayer [159] dielectric mirrors can be deposited onto the fiber by magnetron sputtering. After splicing with another fiber, internal mirrors with reflectance of greater than $85 \%$ can be achieved [159]. Although this technique has shown preliminary success, it is limited by the need for a special coating on the fiber and deterioration in film quality during splicing. Moreover the coupling between the coating and the fiber core may cause additional loss due to surface roughness, cleave angle or reflection into the cladding of the fiber [160]. Another method of producing high-finesse fiber cavities is to combine two fiber Bragg gratings (FBG) as 
mirrors [144]. High finesse values can be achieved with narrow spectral-width FBG [161]. Recently chirped Bragg gratings with much wider spectral widths have been examined in FP filters [162]. Theoretical analyses predict that high finesse and wide spectral widths could be achieved with chirped Bragg gratings [163]. Similar to the fabrication of gratings, a UV laser can cause the changes in refractive index of photosensitive fiber or hydrogen-loaded fiber. The exposed fiber area with a different refractive index works as a reflector in a low-finesse FP cavity. A number of sensors can be easily multiplexed due to the low insertion loss of each FP interferometer [145]. However, the low reflectance and low finesse of UV-induced IFPI sensors require a highpower light source and ultrasensitive detector. In another approach, the reflection between fibers with different core diameters works as a reflective mirror in a costeffective way. But extensive polishing is required to avoid ample power losses [164]. Cleaved fiber ends separated by air gaps can serve as reflectors for intrinsic FP cavity [35, $135,138]$. The cavity size is limited by the coupling efficiency thus reduce the flexibility in fabrication and multiplexing.

In this research, microgap FP sensors were fabricated by combining wet etching and fusion splicing [139]. This process is cost-effective, easy to multiplex and suitable for batch production. Multicavity sensors for thin-film applications were constructed by multiplexing microgap sensors. This approach not only has the advantages similar to the tubing-based MFPI sensors described in Chapter 2, but also excels at its simplicity in fabrication process and flexibility in cavity lengths. Furthermore, the temperature sensitivity for temperature compensation has increased by an order of magnitude from the tubing-based MFPI sensor. Thus the thin-film characteristics under temperature variations could be examined more accurately.

\subsection{Sensor fabrication}

Chemical etching is widely used in fiber optic probe fabrications. Conical core [80], microwells [88], as well as nanotips $[165,166]$ can be achieved with wet-etching techniques. Chemical etching offers a simple and cost-effective fabrication technique, in 
which the optical fibers are dipped into a balanced solution of hydrofluoric acid (HF), and ammonium fluoride $\left(\mathrm{NH}_{4} \mathrm{~F}\right)$ buffer. The process depends on a differential etch rate between the pure silica of the fiber cladding and the germanium-doped silica of the fiber core (SMF-28 Corning). $\mathrm{NH}_{4} \mathrm{~F}$ is used to reduce the dissolution rate of the germaniumdoped core. The chemical reaction of $\mathrm{SiO}_{2}$ and $\mathrm{GeO}_{2}$ with buffered hydrofluoric acid (BHF) can be described by [167]:

$$
\begin{aligned}
& \mathrm{SiO}_{2}+4 \mathrm{HF}+2 \mathrm{NH}_{4} \mathrm{~F} \rightarrow\left(\mathrm{NH}_{4}\right)_{2} \mathrm{SiF}_{6}+2 \mathrm{H}_{2} \mathrm{O}, \\
& \mathrm{GeO}_{2}+4 \mathrm{HF}+2 \mathrm{NH}_{4} \mathrm{~F} \rightarrow\left(\mathrm{NH}_{4}\right)_{2} \mathrm{GeF}_{6}+2 \mathrm{H}_{2} \mathrm{O} .
\end{aligned}
$$

The difference in the solubility of the resultant $\left(\mathrm{NH}_{4}\right)_{2} \mathrm{SiF}_{6}$ and $\left(\mathrm{NH}_{4}\right)_{2} \mathrm{GeF}_{6}$ leads to the differential etching rate between core and cladding [168].

The geometry of etched fiber can be controlled by the doping ratio of $\mathrm{GeO}_{2}$, the composition of the etching solution, and the etching time. Here the $\mathrm{GeO}_{2}$-doping ratio is $3.6 \mathrm{~mol} \%$ which correspond to the refractive index differences of $0.36 \%$ (Corning SMF28). The cone angle of the etched tip is in inverse ratio with volume ratio of $\mathrm{NH}_{4} \mathrm{~F}$ in BHF [168]. Various cone angles can be obtained by controlling the volume ratio of $\mathrm{NH}_{4} \mathrm{~F}$. In contrast to the conventional fiber tip in scanning near-field microscopy (SNOM), etched fiber probes with cone angles larger than $180^{\circ}$ means that the etching rate of the core is larger than that of the cladding. The negative cone angle corresponds to a dip in the core area. After splicing with another fiber, the resulting microgap works as a reflector in the optical-fiber FP cavity. The etching solution used was composed of hydrofluoric acid (HF 50\% weight concentration), ammonium fluoride $\left(\mathrm{NH}_{4} \mathrm{~F} 40 \%\right.$ weight concentration) and deionized water. When the volume ratio $\mathrm{NH}_{4} \mathrm{~F}: \mathrm{HF}: \mathrm{H}_{2} \mathrm{O}=\mathrm{X}: 1: 1$ $(\mathrm{X}<1.6$ [169] or 1.7 [170]), the etching rate of the core will be faster than that of the cladding. Etching rate is also closely related to the temperature of the solution. To avoid the high-temperature use of HF and simplify the procedure, all the experiments were carried out at room temperature $\left(25^{\circ} \mathrm{C}\right)$.

The fiber (corning SMF-28) was first cleaved using a cleaver (Fujikura CT-04B). The reflection intensity of the cleaved fiber was examined by the CTS (Micron Optics SI720) to ensure the quality of the end face. Then the fiber was etched in a BHF solution 
for $15 \mathrm{~min}$. The volume ratio of $\mathrm{BHF}$ can be expressed as $\mathrm{NH}_{4} \mathrm{~F}: \mathrm{HF}: \mathrm{H}_{2} \mathrm{O}=0.5: 1: 1$. After rinsing in deionized water and drying, the end face of the fiber tip was examined under the microscope (Zeiss Axiovert 25). Figure 3.1 shows a picture of the etched end face. By comparing the diameter of the fiber tip before and after etching, the etching rate of the cladding can be calculated as $1.2 \mu \mathrm{m} / \mathrm{min}$. The etching rate of the core is a little bit higher than that of the cladding so there is a dip in the core area which is shown in Figure 3.1. The thickness of the dip is about 1-2 $\mu \mathrm{m}$, as estimated under the microscope. The morphology of the cladding and the dipped core are not purely flat, which may come from the composition profile during the preform fabrication [170]. Generally, this could be eliminated by carefully controlling the preform fabrication conditions.

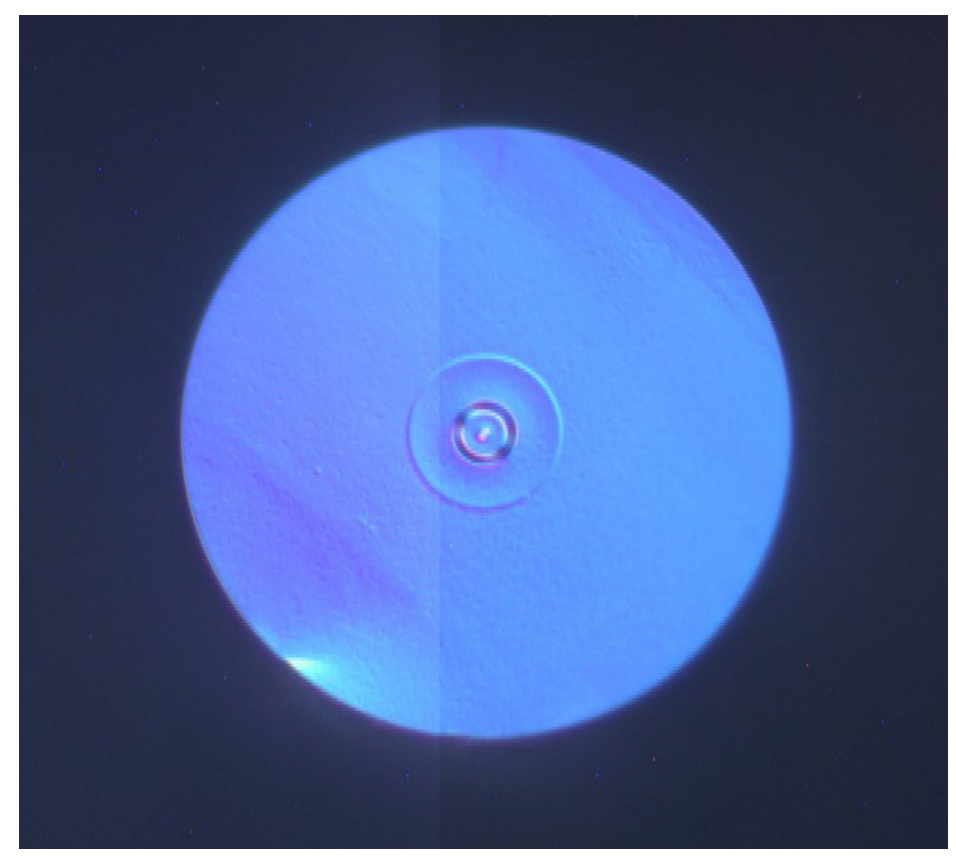

Figure 3.1 The end face of a fiber (corning SMF-28) after 15 min etching in BHF.

The etched fiber was then spliced with another SM fiber using a conventional fiber splicer (Sumitomo Type-36). A microgap was formed in the core area between the fibers and behaves as a reflector in a FP cavity. The splicing conditions are similar to that of the tubing-based MFPI sensor (Table 2.1). Shorter arc time and lower arc power are 
preferred during splicing. Otherwise the reflectance from the microgap will become lower or even disappear. By cleaving the reflection fiber, the endface acts as another reflector of the FP cavity. A low-finesse FP cavity was thus formed at the end of the fiber (Figure 3.2). Figure 3.3 is the picture of a microgap sensor under the microscope. Due to curvature in the etched core area, the reflection at that surface is much lower than the cleaved end. Normally a $5 \mathrm{~dB}$ decrease in the reflection intensity is seen at the etched fiber tip compared with a cleaved fiber. The microgap FP sensor can be analyzed as a single FP cavity sensor (Figure 3.2).

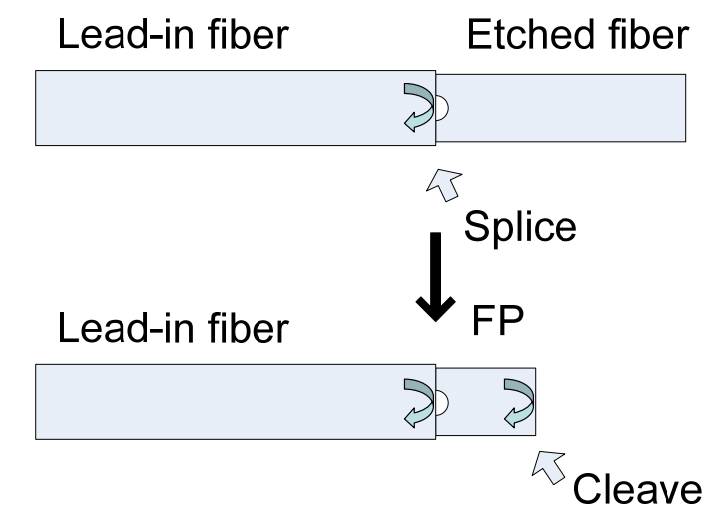

Figure 3.2 Fabrication of microgap FP sensors.

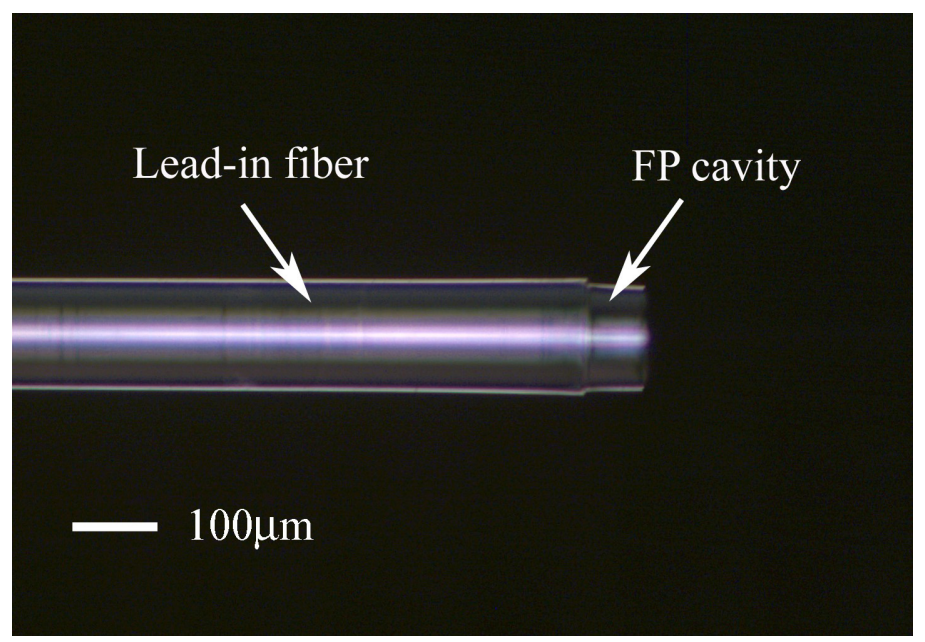

Figure 3.3 Picture of a microgap FP sensor. 
That single-cavity FP sensor itself can work as a thin-film sensor in chemical sensing or biosensing by monitoring the changes in the reflection spectrum during adsorption. Moreover, the microgap sensor is distinguished for its multiplexing capability. By multiplexing with another microgap sensor, temperature and thin-film adsorption can be examined simultaneously. This not only reduces the temperatureinduced error but also provides the opportunity to investigate the film under temperature variations.

By splicing a microgap sensor with another etched fiber, a second microgap was formed between the sensor and the etched fiber. A multicavity sensor was fabricated by cleaving the second etched fiber. Figure 3.4 illustrates the structure of the multicavity sensor. A picture of a multicavity sensor is shown in Figure 3.5. The two FP cavities are designed with different purposes. The first FP cavity (FP1 in Figure 3.4) provides the temperature sensing capability and the second cavity (FP2 in Figure 3.4) offers the thinfilm sensing functionality.

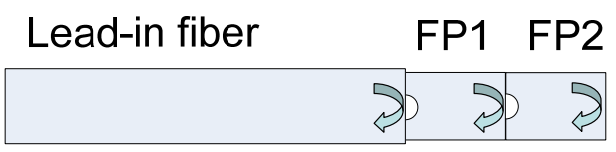

Figure 3.4 Structure of the microgap multicavity FP sensor. 


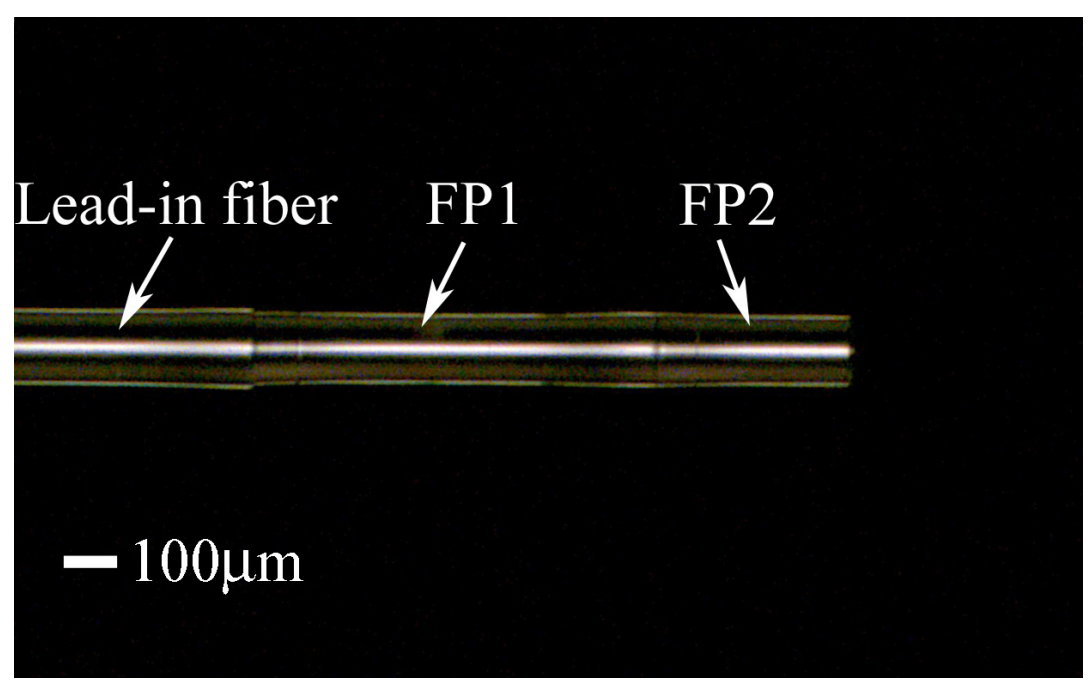

Figure 3.5 Picture of a microgap multicavity FP sensor.

\subsection{Signal analysis}

\subsubsection{Single-cavity sensor}

The microgap sensor in Figure 3.2 can be simplified as a single-cavity sensor due to the much lower reflection from the etched surface. Figure 3.6 gives a sample spectrum of a microgap FP sensor in the wavenumber domain. The sinusoidal waveform validates our assumption. The Fourier transformation of the reflection spectrum is given in Figure 3.7. The low frequency component is mainly due to the DC offset in the reflection spectrum. A band pass filter is able to efficiently separate the fiber cavity signal from the lower frequency signal and higher frequency harmonics [171]. Finite impulse response (FIR) filters were selected due to the linear phase response to the frequency. Figure 3.8 shows the Fourier transformation of the bandpass-filtered spectrum. The single frequency signal has been separated by the filter. The phase delay of an Nth-order filter can be expressed as [172]

$$
n=\frac{N-1}{2}
$$


The delay can be easily compensated by shifting the filtered signal backward by $n$ samples.

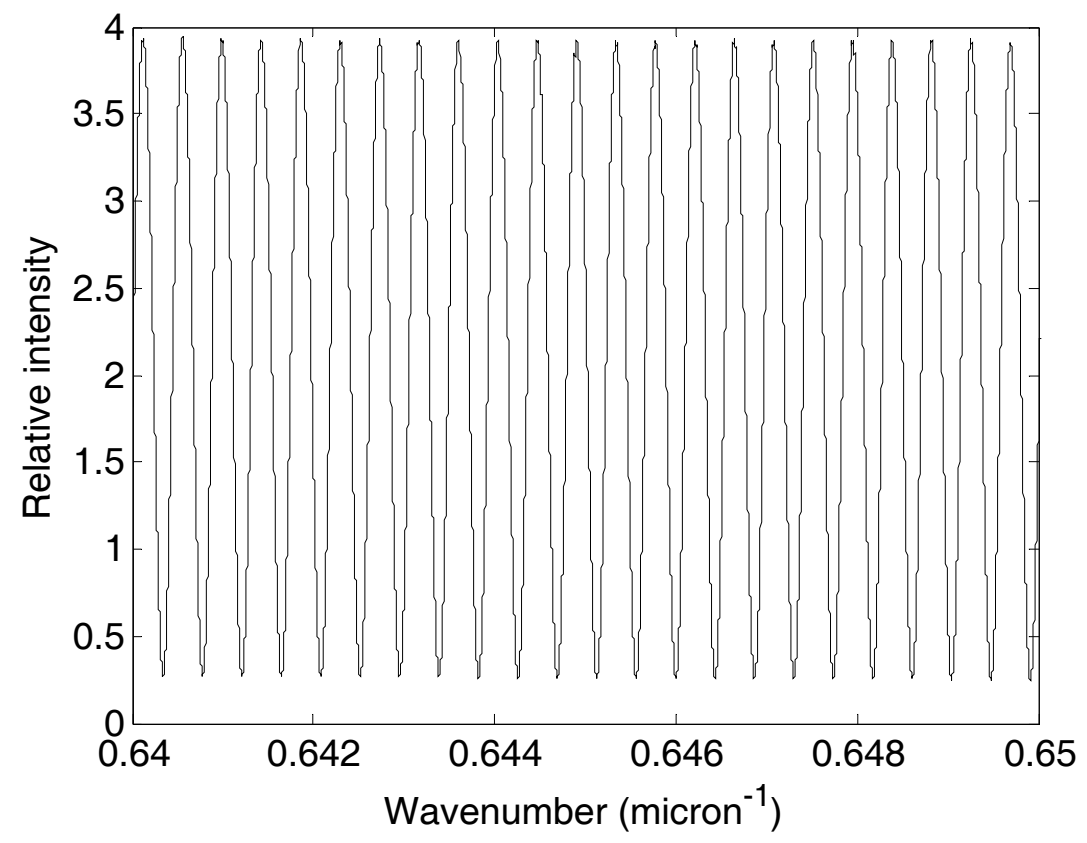

Figure 3.6 Reflection spectrum of a microgap FP sensor.

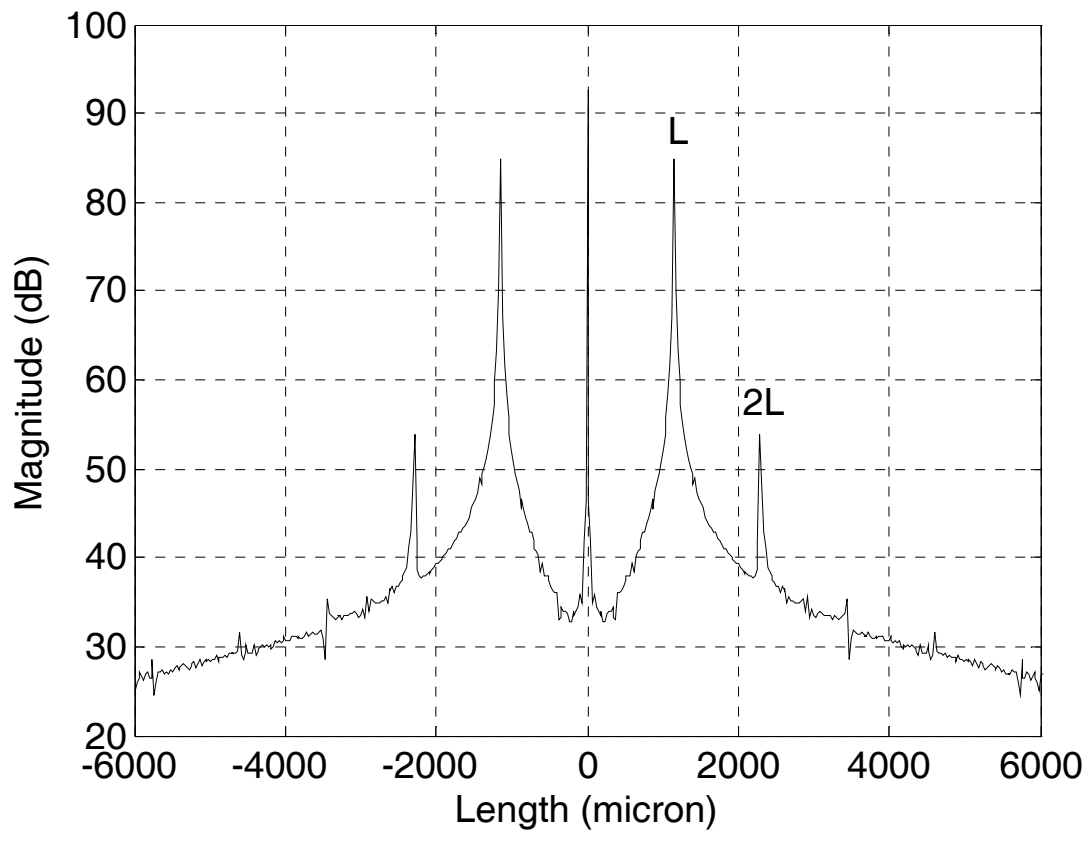

Figure 3.7 Fourier transformation of the reflection spectrum. 


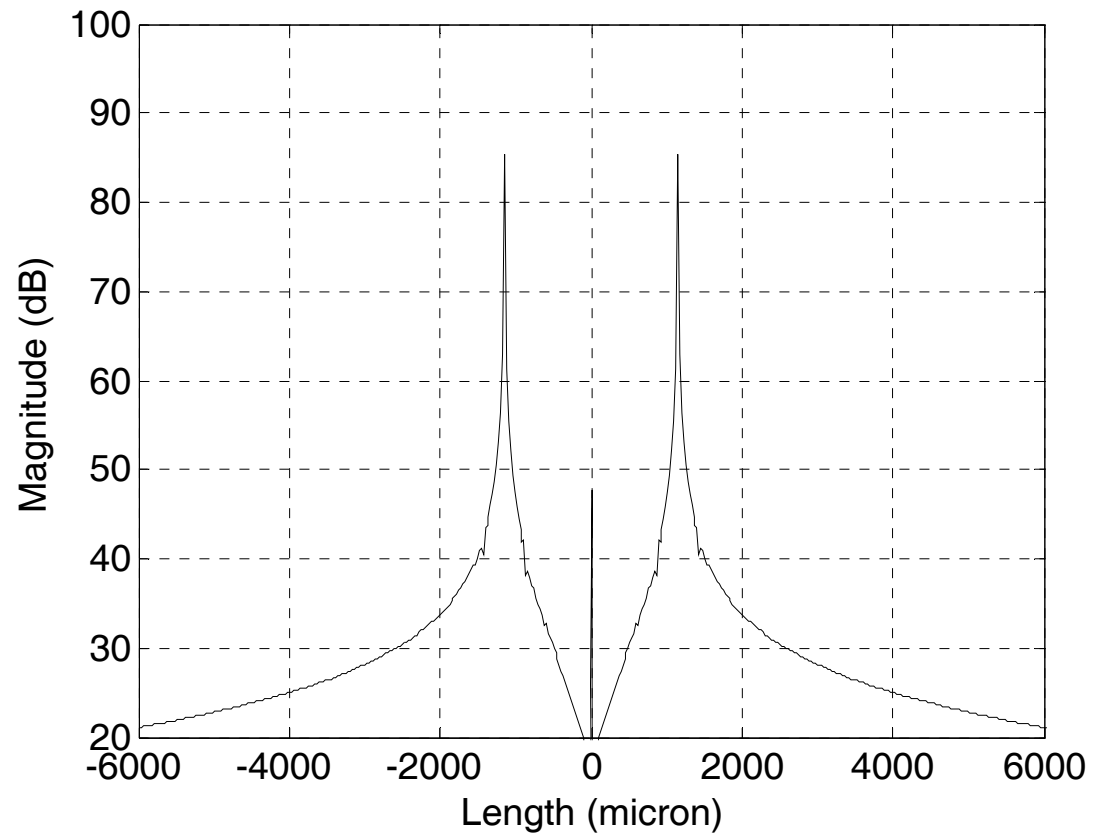

Figure 3.8 Fourier transformation of the bandpass-filtered spectrum.

The reflection spectrum of a low-finesse single-cavity FP sensor can be given as

$$
I=A+B \cos (2 K L+\phi),
$$

where $A$ and $B$ are coupling coefficients and independent of wavelength, $K=2 \pi / \lambda$ is the circular wavenumber, $L=n d$ is the optical thickness of the FP cavity, and $\phi$ is the coupling phase shift. As introduced in section 2.3.1, the combination of single-peak (single-valley) tracing and multiple-peak (multiple-valley) provides large dynamic range and high resolution simultaneously. The first step is to determine the specific order number of a certain peak. From Eq 3.3, the adjacent peaks satisfy

$$
\begin{aligned}
2 K L+\phi & =2 \pi m, \\
2 K^{\prime} L+\phi & =2 \pi(m+1),
\end{aligned}
$$

where $m$ is the order number of a peak and should be a positive integer. The order number can be expressed as: 


$$
m=\left[\frac{K}{\Delta K}+\frac{\phi}{2 \pi}\right],
$$

where the square brackets mean to round towards the nearest integer, and $\Delta K=K^{\prime}-K$. Here the phase shift $\phi$ is from the coupling of light in the microgap. According to Eq 2.16, the phase shift is related to the size of the microgap. The size of the microgap is only about 1-2 $\mu \mathrm{m}$, so the phase shift will not affect the rounding result in calculation of the order number. Then the optical cavity length (OCL) can be calculated as:

$$
L=\frac{2 \pi m-\phi}{2 K} \text {. }
$$

It is difficult to derive the exact cavity length from above equation because of the unknown phase shift from the microgap. The optical cavity length can be estimated as

$$
L \approx \frac{\pi m}{K}=\frac{m \lambda}{2}
$$

since $\phi<<2 \pi m$. The error of this estimation depends on the phase shift. In a rough estimation, a $0.1 \mathrm{rad}$ phase shift will cause an optical-cavity-length error of $0.01 \mu \mathrm{m}$ $(\lambda=1.55 \mu \mathrm{m})$. However, we care more about the relative changes of the optical cavity length than the absolute cavity length itself. It is possible to calculate the changes in the optical cavity length as

$$
\Delta L=\frac{2 \pi m-\phi}{4 \pi} \Delta \lambda \approx \frac{m}{2} \Delta \lambda,
$$

where $\Delta \lambda$ is the wavelength shift of the $m$ th order peak. The relative error is

$$
\frac{\delta(\Delta L)}{\Delta L}=\frac{\frac{\phi}{4 \pi} \Delta \lambda}{\frac{m}{2} \Delta \lambda}=\frac{\phi}{2 \pi m}<<1 .
$$

For a FP cavity with OCL of $200 \mu \mathrm{m}$, the order number of a peak at $\lambda=1550 \mathrm{~nm}$ is $m=2 L / \lambda \approx 258$. If the phase shift is $0.1 \mathrm{rad}$, the relative error of the changes in OCL is only $6 \times 10^{-5}$. In our study, the change in OCL is always less than $1 \mu \mathrm{m}$, so the error will be smaller than $0.1 \mathrm{~nm}$. 


\subsubsection{Multicavity sensor}

A multicavity sensor with structure shown in Figure 3.4 is constructed by two FP cavities. Figure 3.9 shows a reflection spectrum of that kind of sensor. The Fourier transformation in Figure 3.10 clearly illustrates the spatial frequency components from each FP cavity and their combinations. Following a similar method as for the singlecavity sensor, the frequency component of each cavity can be filtered out by specially designed bandpass filters. Then the cavity lengths and their changes can be calculated as described in last section.

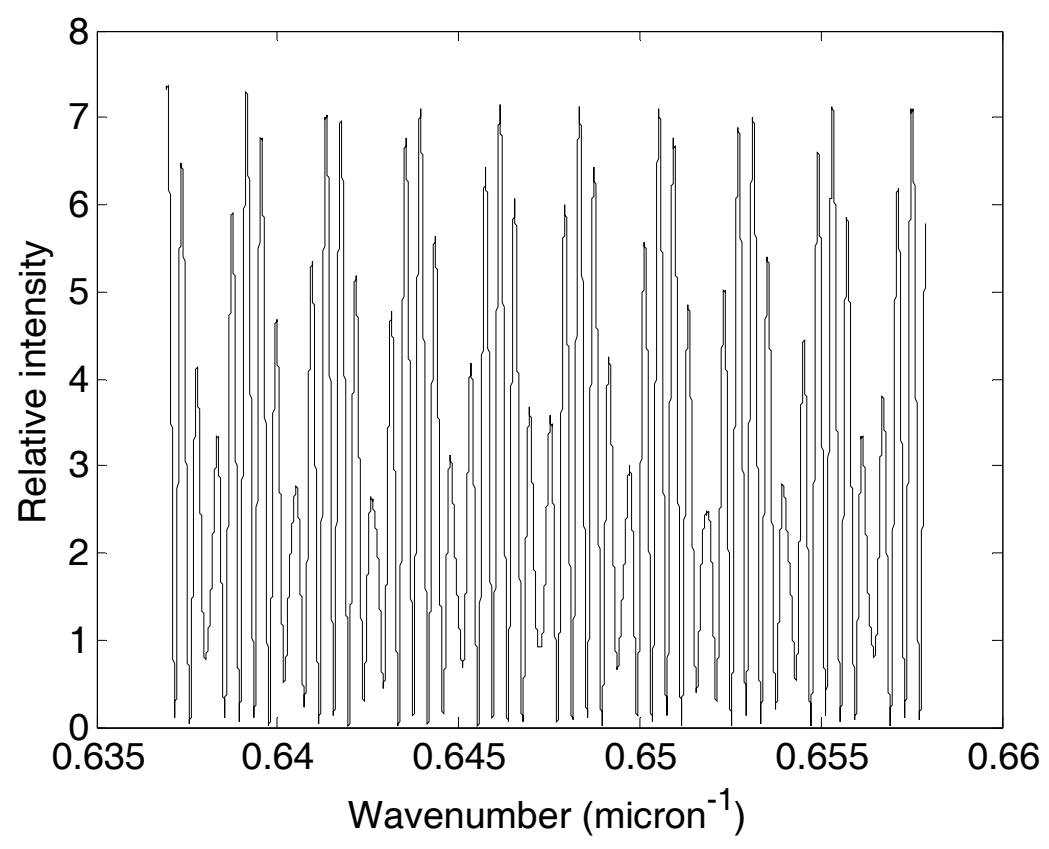

Figure 3.9 Reflection spectrum of a microgap multicavity FP sensor. 


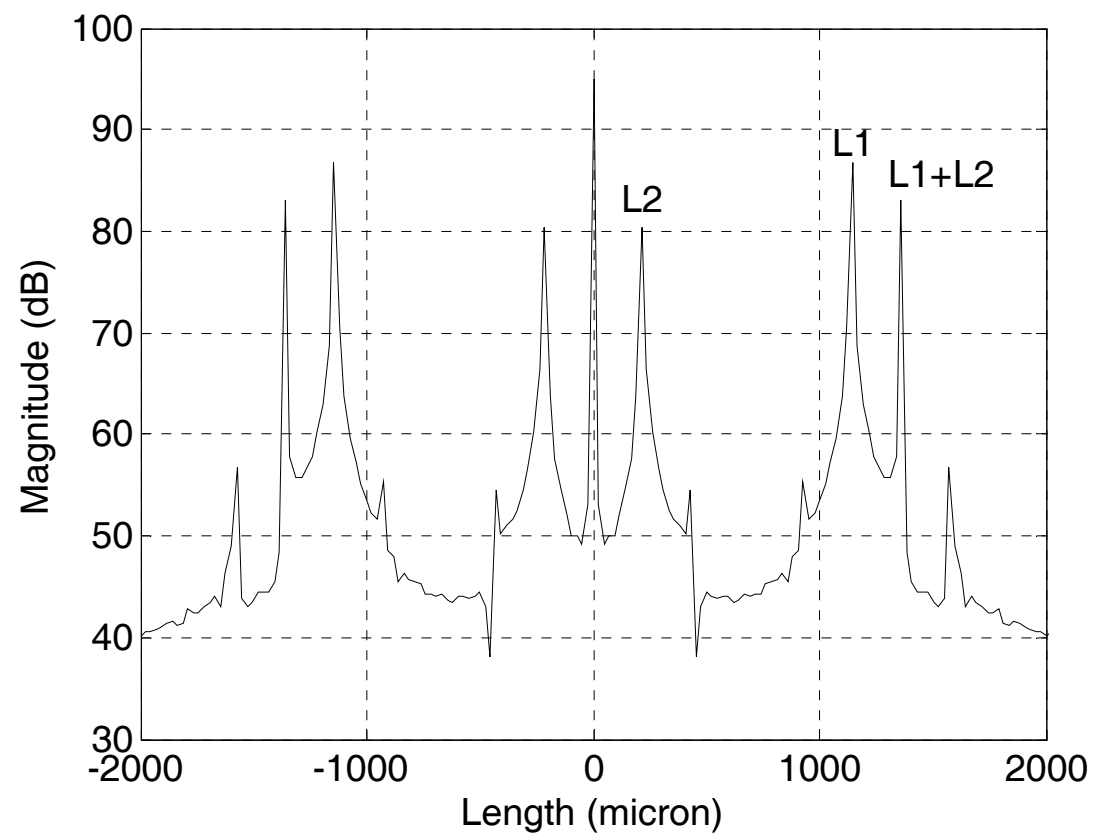

Figure 3.10 Fourier transformation of the reflection spectrum of a microgap multicavity FP sensor.

\subsection{Temperature compensation}

The microgap sensor has been used in temperature, strain and thin-film measurement [139]. The sensor is distinguished by its simplicity, low cost and high sensitivity in monitoring various changes. It is capable of measuring ultra-thin films in chemical or biological applications by comparing the changes in the optical thickness of the cavity. However, the thermal-induced errors can not be neglected due to the significant influence on the measurement accuracy. It is necessary to introduce temperature compensation in high-accuracy measurements at varying temperatures. In tubing-based MFPI sensors, temperature compensation was achieved by extracting the temperature information from the air cavity. The temperature resolution is about $2^{\circ} \mathrm{C}$ because of the low thermal expansion coefficient. The microgap multicavity sensor increases the temperature sensitivity by an order of magnitude, simplifies the fabrication procedures and provides flexibility in the cavity lengths. 
The experimental setup of the microgap sensor system is shown in Figure 3.11. A low-noise fiber ring laser from a component testing system (CTS Micron Optics) was coupled into the sensor. The reflection spectrum of the sensor was monitored by the detector in the CTS and analyzed by a personal computer. The CTS offers a measurement range of 1520-1570 $\mathrm{nm}$ with $1 \mathrm{pm}$ accuracy.

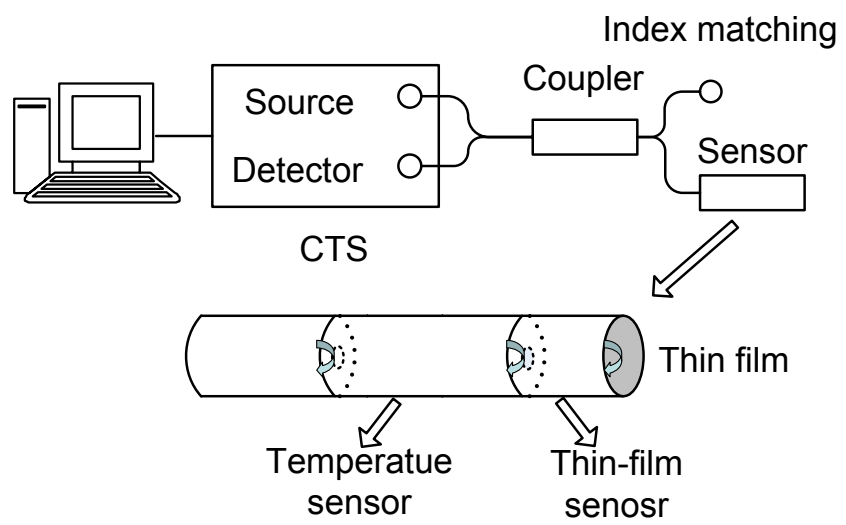

Figure 3.11 Schematic of microgap multicavity FP sensor system for thin-film applications

A multicavity sensor was constructed with a temperature sensor and a thin-film sensor as in Figure 3.11. Similar to the description in section 2.4, the optical thickness of the thin-film sensing cavity is also highly dependent on the environment temperature due to the thermo-optic effect and thermal expansion. The change in the optical thickness of the cavity with temperature can be expressed as Eq. 2.53. The effective temperature coefficient is about $7.4 \times 10^{-6} /{ }^{\circ} \mathrm{C}(\lambda=0.633 \mu \mathrm{m})$. For a FP sensor with a $100 \mu \mathrm{m}$ fiber cavity, the variation in optical thickness over a $100^{\circ} \mathrm{C}$ temperature range is around 100 $\mathrm{nm}$, which is too large for thin-film measurement. The thermal effect can be reduced by shorter cavity length. As shown in Figure 3.12, an optical cavity length as small as $20 \mu \mathrm{m}$ can be achieved by cleaving under a microscope. However, the difficulty in obtaining high-visibility fringes increases when the size of the cavity becomes shorter. Furthermore, a light source with wider wavelength range is required in demodulation of the reflection spectrum. From Eq. 2.51, the wavelength range can be expressed as 


$$
\Delta \lambda \approx \frac{\lambda^{2}}{2 L}
$$

where $\lambda$ is the central wavelength of the light source, $L=n d$ is the optical cavity length. For a FP cavity with optical cavity length as small as $10 \mu \mathrm{m}$, the wavelength range of the light source $(\lambda=1550 \mathrm{~nm})$ should be larger than $120 \mathrm{~nm}$ for multiple-peak demodulation. Due to the difficulties existing in both fabrication and signal processing, a single-cavity sensor is not appropriate for thin-film measurement under temperature variations. A microgap multicavity sensor with a built-in temperature sensor and thin-film sensor not only compensates the temperature-induced error but also simplifies the fabrication and signal analysis compared to tubing-based MFPI sensors. To effectively demodulate the reflection spectrum of the multicavity sensor, two cavities are fabricated with different thickness. The temperature-sensing cavity is longer for better temperature sensitivity which depends on the relative changes in the optical thickness of the cavity. The thinfilm-sensing cavity is shorter for higher resolution of the absolute changes in optical thickness during adsorption. Here the optical cavity lengths of the temperature sensor and thin-film sensor are around $1000 \mu \mathrm{m}$ and $200 \mu \mathrm{m}$, respectively. The lengths of each cavity are quite flexible as long as their corresponding frequency components can be effectively differentiated from each other. 


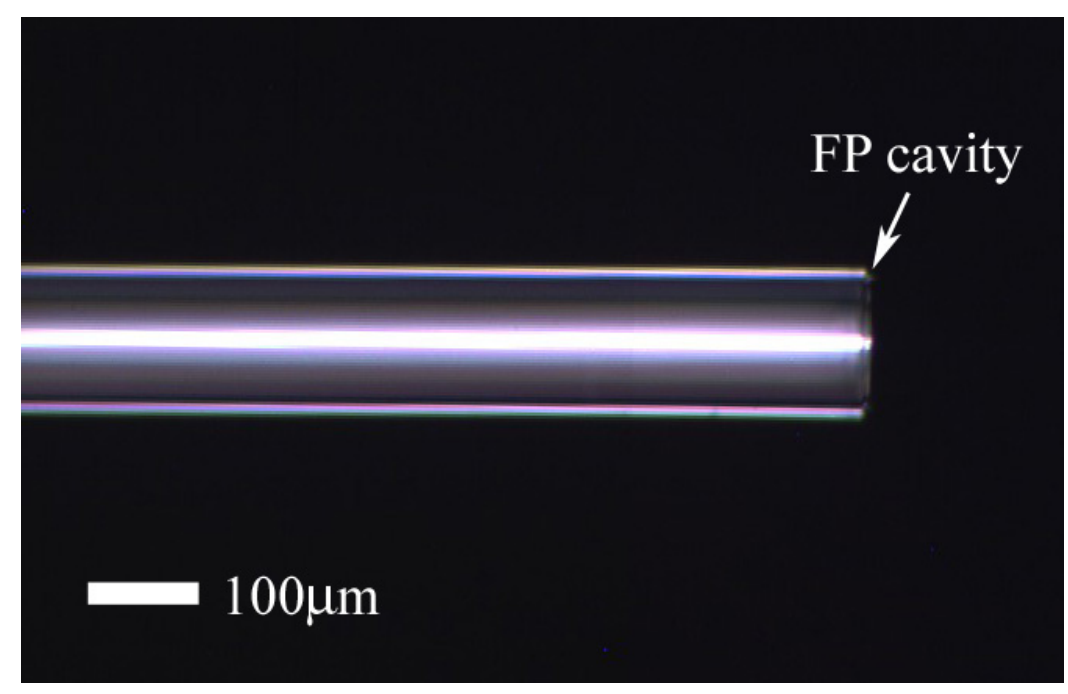

Figure 3.12 A microgap sensor with small FP cavity.

The multicavity sensor was calibrated in an environmental chamber (1020C TestEquity). The temperature response of each cavity is illustrated in Figure 3.13 and Figure 3.14. The relationship between the optical cavity length and temperature is almost linear, as expressed in Eq. 2.53. The weak nonlinearity may come from the thermal expansion effect of silica [157]. According to Eq. 2.53 and 2.54, the changes in optical cavity length can be derived as:

$$
\Delta L=\left[\left(\frac{\alpha_{n}}{n}+\alpha_{d}\right) \Delta T+\beta_{d} \Delta T^{2}\right] L,
$$

where $\alpha_{n}$ is the thermo-optic coefficient, $\alpha_{d}$ and $\beta_{d}$ are the thermal expansion coefficient. The temperature sensitivity of the microgap sensor is related to the thermo-optic effect and thermal expansion of the FP cavity. The temperature sensitivity of the tubing-based sensor in Chapter 2 is based on the thermal expansion of the silica tubing. The thermooptic coefficient of silica is more than ten times larger than the thermal expansion coefficient [156]. So the temperature sensitivity of the microgap sensor is higher than the tubing-based FP sensor by an order of magnitude. Thus the thin-film characteristics under temperature variations could be examined more accurately. 


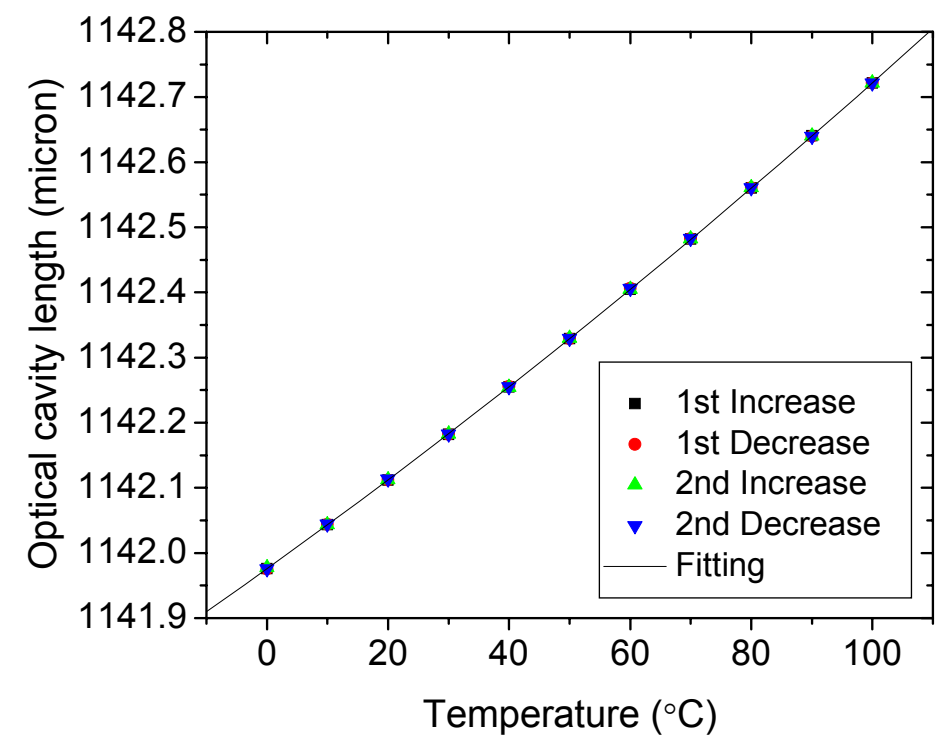

Figure 3.13 Changes in optical cavity length of temperature sensor with temperature from 0 to $100^{\circ} \mathrm{C}$.

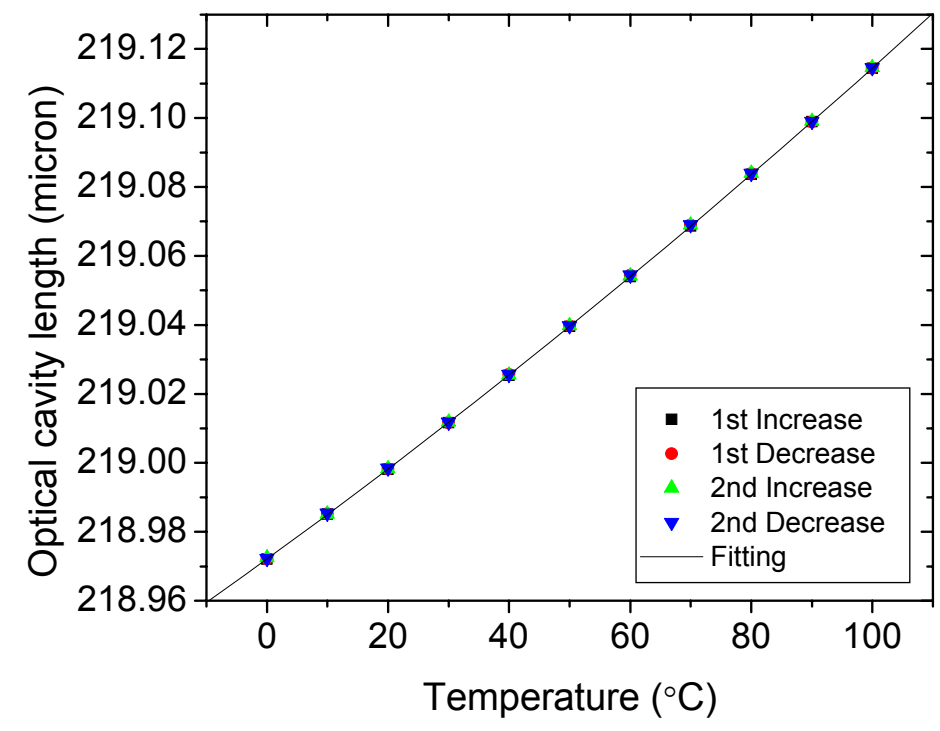

Figure 3.14 Changes in optical cavity length of thin-film sensor with temperature from 0 to $100^{\circ} \mathrm{C}$. 
The relative changes in the optical cavity lengths of the temperature sensor and thin-film sensor can be given as:

$$
\frac{\Delta L_{f}}{\Delta L_{T}}=\frac{L_{f}}{L_{T}},
$$

where $L_{f}$ and $L_{T}$ are the optical cavity lengths of each cavity. Figure 3.15 shows the linear relationship between the changes of the cavities. Therefore the thermal-induced error in thin-film sensing can be easily compensated by comparing the changes in optical cavity lengths of two sensors. Figure 3.16 shows the temperature compensation effect on the optical thickness error of the thin-film sensor before adsorption. In the temperature range from 0 to $100^{\circ} \mathrm{C}$, the temperature dependence is about $\pm 70 \mathrm{~nm}$ before compensation. The thermal-induced error greatly affects the thin-film measurement under temperature variations. Temperature compensation effectively reduces the thermal-induced error to less than $\pm 0.2 \mathrm{~nm}$ over the entire temperature range.

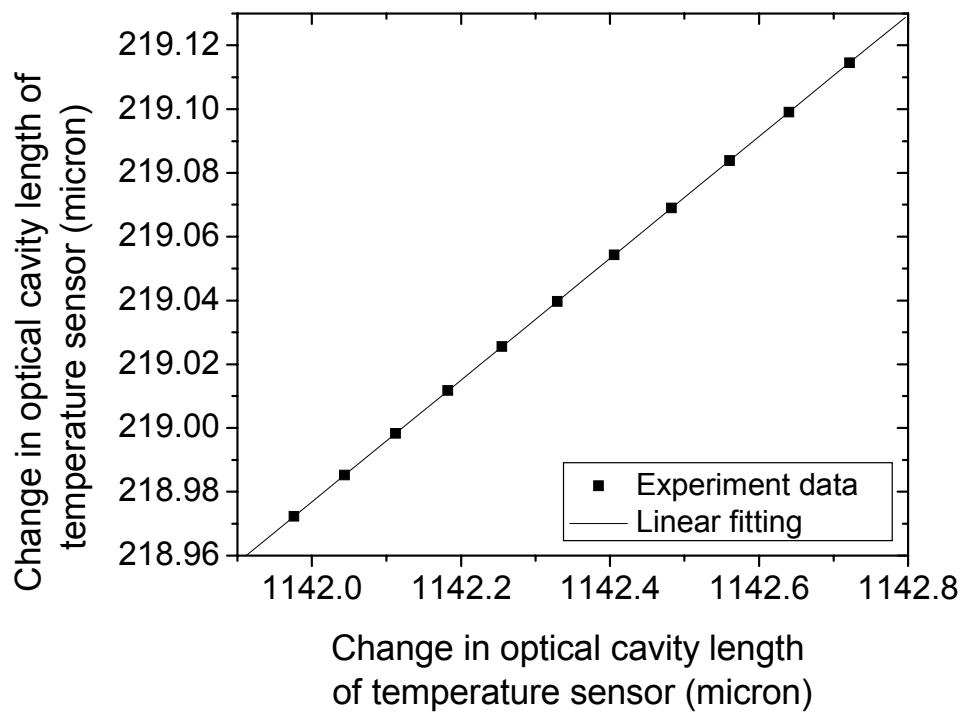

Figure 3.15 Comparison of changes in optical cavity lengths of temperature sensor and thin-film sensor with respect to temperature from 0 to $100^{\circ} \mathrm{C}$. 


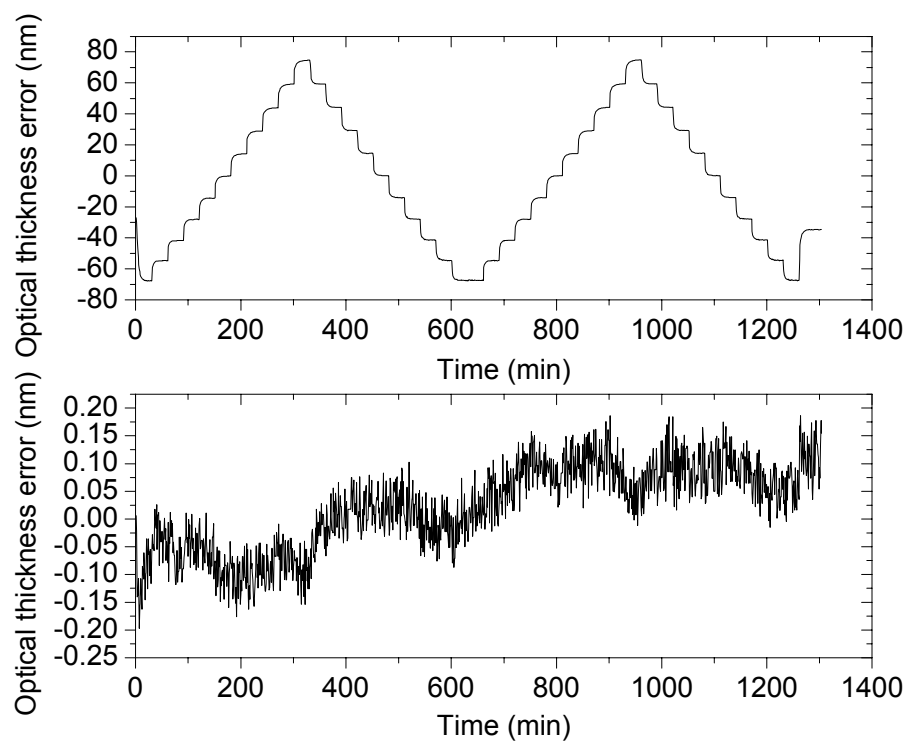

Figure 3.16 Effects of temperature compensation on the optical thickness error of thinfilm sensor. The temperature cycled twice by steps of $10^{\circ} \mathrm{C}$ in the range from 0 to $100^{\circ} \mathrm{C}$. The upper curve shows the optical thickness error before compensation and the lower curve shows the error after compensation.

To demonstrate the effect of temperature compensation in thin-film sensing, the multicavity sensor was coated with a self-assembled polyelectrolyte multilayer. The sensor was alternately immersed in a polycationic (PAH) and a polyanionic (PSS) solution. A multilayer thin film with structure of $[\mathrm{PAH} / \mathrm{PSS}]_{10}$ was self-assembled onto the sensor end. The film thickness was measured to be $86 \mathrm{~nm}(n=1.54)$ at room temperature $\left(25^{\circ} \mathrm{C}\right)$ by calculating the changes in optical cavity lengths of the thin-film sensor. The thin-film was examined in the chamber under temperature range of 0 to $100^{\circ} \mathrm{C}$. Figure 3.17 illustrates the changes in the optical thickness of the thin film. The film was heated in $10^{\circ} \mathrm{C}$ increments, and equilibrated for $30 \mathrm{~min}$ at each step. From 0 to $20^{\circ} \mathrm{C}$, the optical thickness decreased slowly. From 20 to $100^{\circ} \mathrm{C}$, the decrease became more rapidly. Then the film was cooled to $0^{\circ} \mathrm{C}$ in $10^{\circ} \mathrm{C}$ steps and 30 min equilibration time. The optical thickness slowly increased but apparent hysteresis was observed. After the film was kept at room temperature for 24 hours, the optical thickness recovered to its 
initial value. The decrease in the optical thickness and the hysteresis may come from the release of bound water molecules from the film and reentry from the air due to temperature and humidity changes [158].

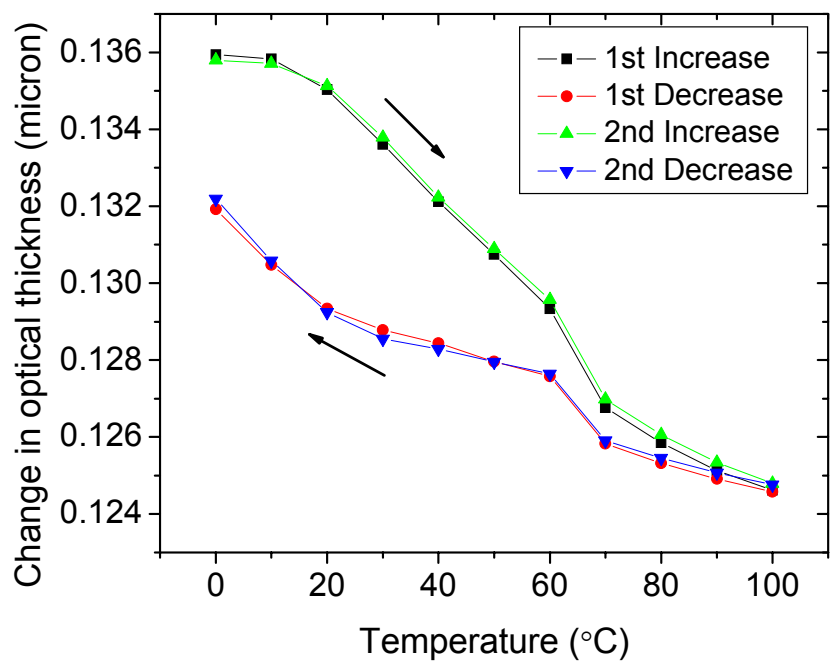

Figure 3.17 Change in optical thickness of the $[\mathrm{PAH} / \mathrm{PSS}]_{10}$ film under temperature variations.

\subsection{Conclusion}

Microgap FP sensors have been developed by wet etching and splicing techniques. Small dips in fiber cores can be fabricated by differential etching. By splicing the etched fiber with another fiber, a microgap was generated inside the fiber which functioned as a reflector. Low-finesse FP cavities were formed between the microgap reflectors or cleaved fiber ends. This process provides a simple and cost-effective method for IFPI sensor fabrication. The multiplexing of microgap sensors with other sensors provides an effective method of temperature-compensated measurement.

Multicavity sensors for thin-film measurement were constructed by multiplexing microgap sensors. It not only has advantages similar to tubing-based MFPI sensors, but also excels at its simplicity in fabrication process and flexibility in cavity lengths. 
Furthermore, the temperature sensitivity for temperature compensation has increased by an order of magnitude from the tubing-based MFPI sensor. Thus the thin-film characteristics under temperature variations could be examined more accurately. 


\section{Chapter 4 Surface modification by polymer self-assembly}

\subsection{Introduction}

The performance of a biosensor is closely related to the activity of the biosensing molecules. Biological molecules are highly efficient at recognizing specific analytes or catalyzing reactions, but only in specific aqueous media, which limits their applications in biosensor development. The lack of a preferred medium often results in denaturation and loss of reactivity in many cases where biomolecules have been directly bound to solid surfaces through adsorption. Immobilization techniques to stabilize and preserve the reactivities of biomolecules are currently being pursued in biosensing. Thin organic films for supporting or embedding the biomolecules are of special interest. Such thin films have been fabricated by Langmuir-Blodgett (LB) or self-assembly techniques [173, 174]. Multilayer protein films have been formed by electrostatic layer-by-layer adsorption of oppositely charged polyelectrolytes and proteins [174]. It was demonstrated that polymer self-assembly is an effective method for immobilization of biological molecules in biosensing [175].

Electrostatic self-assembly (ESA), a low-cost and simple nanofabrication technique, has been widely investigated $[176,177]$. The growth process is quick with a few minutes per layer, and fabrication is achieved at room temperature. It is easy to control the detailed structure and thickness. ESA films are naturally self-limiting in thickness and uniform at the molecular level. Therefore, film thickness increases linearly with the number of bilayers and each bilayer has uniform thickness. The monolayers are formed by electrostatic attraction between oppositely charged organic polyelectrolytes: polycations (positively charged) and polyanions (negatively charged). As shown in Figure 4.1, alternating adsorption of cationic and anionic monolayers onto a charged substrate allows multilayer structures to be formed electrostatically in nanometer steps. The film structure can be controlled by an array of parameters such as polymer concentration, ionic strength, $\mathrm{pH}$ and substrate conditions. For example the film thickness can be controlled by the salt concentration in the polymer solutions [178]. Not only the 
deposition conditions but also environmental factors such as temperature and humidity may also affect the film thickness $[158,179]$. The interfaces in the multilayer structure are ambiguous because of the interpenetration [180]. Under certain conditions, the multilayer film can be simplified as a single homogeneous film.

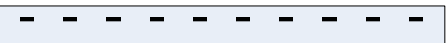

(a)

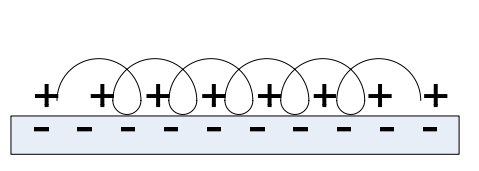

(b)

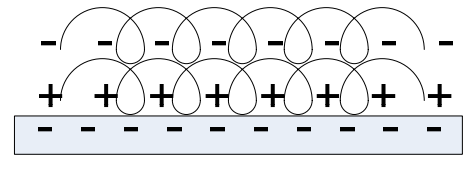

(c)

Figure 4.1 Adsorption steps of thin film deposition. The polycation adsorption and polyanion adsorption steps can be repeated to the required layer numbers. (a) Negative charged substrate; (b) Polycation adsorption; (c) Polyanion adsorption.

The contribution from the electrostatic effect also depends on the chemical nature of the polyions. The electrostatic attraction may be the major driving force for multilayer buildup. However, there are many other interactions that can be used for multilayer deposition such as hydrogen bonds [181], covalent bonds [182] and specific recognition [183]. Thus various materials can be incorporated into the multilayer films such as small molecules [184], macromolecules [158], proteins and DNA [175, 185-187]. Polymer selfassembly has been used as substrates for immobilization of immunoglobulin $\mathrm{G}(\mathrm{IgG})$ and anti-IgG [175]. The immobilized IgG (anti-IgG) layers remain active to their biospecific partner species.

ESA is also an effective technique in surface modification. The silica surface of the optical fiber is known to be able to adsorb proteins. However, proteins are denatured (the hydrogen-bonded configuration is broken) by the forces that flatten the molecules against the surface [131]. Therefore, polymer self-assembly is necessary to retain activity of biological molecules in our fiber optic biosensors. Furthermore, it increases the capacity of the sensing thin film through the fabrication of a multilayer antibody film. 


\subsection{Characterization of polymer self-assembly by ellipsometry}

Polymer self-assembly has been investigated by ellipsometry [188], x-ray reflectometry [176], near-field scanning optical microscopy [189], atomic force microscopy [190] and other techniques [190-192]. Some of them can be applied as in situ characterization methods to analyze the kinetics of adsorption [188, 190-192]. Among those techniques, ellipsometry is a versatile optical technique that has applications in many different fields. Ellipsometry measures the changes in polarization of light upon reflection from a sample. The change in polarization is determined by the thickness and refractive index of the sample. It is commonly used to characterize thin films with an excellent accuracy.

\subsubsection{Analysis of factors on thin-film characteristics}

Thin-film sensing by multicavity FP sensor is based on the measurement of optical thickness changes upon the adsorption of molecules. Optical thickness is the product of the thickness and refractive index. Previous works have shown that deposition conditions and environmental factors affect both of the characteristics [158, 178, 179, 193]. The factors affect $\mathrm{S} / \mathrm{N}$ ratios and mean values simultaneously. $\mathrm{S} / \mathrm{N}$ ratios correspond to the repeatability in characteristics. Mean values varies across the levels of factors which shows the relative contribution of each factor. In the multicavity FP biosensor, polymer self-assembly works as the precursor film. The variation of the polymer film between each self-assembling process may affect the subsequent adsorption of antibody as well as the repeatability in immunosensing. In order to improve the repeatability in polymer self-assembly, high $\mathrm{S} / \mathrm{N}$ ratios for both thickness and refractive index are preferred. A systematic study of the multiple factors was performed in order to provide the optimum conditions for fabrication of the self-assembly. Design of experiments was applied to increase the efficiency of the experimentation. 


\subsubsection{Experimental design}

Experimental design is used to organize the experiment properly to ensure that the right type of data, and enough of it, is available to answer the questions of interest as clearly and efficiently as possible. Since we are investigating the effects of several factors, it is usually more efficient to manipulate these variables in one experiment than to run a separate experiment for each variable. Moreover, only in experiments with more than one independent variable is it possible to test for interactions among variables. By choosing a design with all possible combinations, called a full factorial design, all effects are distinguishable from other effects. However, meaningful results can also be obtained by doing fewer runs or combinations called fractional factorial designs.

Preliminary results show that five primary factors may affect the characteristics of the thin film including the $\mathrm{pH}$ value of the cationic and anionic solutions, the salt concentration in those solutions, the polymer concentration, the polymer species, and the immersion time. To simplify the procedure, each factor was examined at two levels as shown in Table 4.1. Interactions between factors were not investigated to reduce the required number of trials. With an L-8 array, a fractional factorial design with 5 factors and 8 runs is more appropriate than the full factorial design. $\mathrm{S} / \mathrm{N}$ analysis can be obtained by fabricating two identical samples in each trial. Table 4.2 shows the sample matrix of the fractional factorial design.

Table 4.1 Factors and levels for experimental design.

\begin{tabular}{|l|c|c|}
\hline Factor & Low setting & High setting \\
\hline $\mathrm{pH}$ & 6 & 7.5 \\
\hline Salt concentration $(\mathrm{M})$ & 0 & 0.15 \\
\hline Polymer concentration $(\mathrm{mg} / \mathrm{ml})$ & 2 & 4 \\
\hline Cationic polymer species & PAH & PEI \\
\hline Immersion time (min) & 1 & 3 \\
\hline
\end{tabular}


Table 4.2 Sample matrix

\begin{tabular}{|c|c|c|c|c|c|}
\hline Trial No & PH & $\begin{array}{c}\text { Salt } \\
\text { concentration } \\
(\mathrm{M})\end{array}$ & $\begin{array}{c}\text { Polymer } \\
\text { concentration } \\
(\mathrm{mg} / \mathrm{ml})\end{array}$ & $\begin{array}{c}\text { Polymer } \\
\text { species }\end{array}$ & $\begin{array}{c}\text { Immersion } \\
\text { time (min) }\end{array}$ \\
\hline 1 & 6.0 & 0.00 & 2 & PAH & 1 \\
\hline 2 & 6.0 & 0.00 & 2 & PEI & 3 \\
\hline 3 & 6.0 & 0.15 & 4 & PAH & 1 \\
\hline 4 & 6.0 & 0.15 & 4 & PEI & 3 \\
\hline 5 & 7.5 & 0.00 & 4 & PAH & 3 \\
\hline 6 & 7.5 & 0.00 & 4 & PEI & 1 \\
\hline 7 & 7.5 & 0.15 & 2 & PAH & 3 \\
\hline 8 & 7.5 & 0.15 & 2 & PEI & 1 \\
\hline
\end{tabular}

\subsubsection{Materials and methods}

Silicon substrates were cleaned by piranha solution $\left(\mathrm{H}_{2} \mathrm{O}_{2}: \mathrm{H}_{2} \mathrm{SO}_{4}=30: 70 \mathrm{v} / \mathrm{v}\right)$ at room temperature for $1 \mathrm{~h}$, after which the substrates were ultrasonically rinsed in ultrapure water and dried under nitrogen. The ultrapure water used for all experiments was obtained by reverse osmosis (Barnstead Diamond RO) followed by ion exchange and filtration (Barnstead Nanopure Diamond UV/UF). Polyallylamine hydrochloride (PAH $\mathrm{MW} \approx 70000$ ), polysodium 4-styrenesulfonate (PSS MW 70000 ), and polyethylenimine (PEI MW $\approx 25000)$, were obtained from Aldrich. Polymer solutions of the given concentrations were prepared using ultrapure water. $\mathrm{NaCl}$ (Mallinckrodt) was used to adjust the salt concentration. $\mathrm{pH}$ values were adjusted using $\mathrm{HCl}$ and $\mathrm{NaOH}$ by a Corning 455 Ion Analyzer with ATC probe.

Silicon substrates are naturally covered by a thin silica layer $(\sim 2 \mathrm{~nm})$. Silica surfaces immersed in water or solutions are negatively charged primarily through the dissociation of terminal silanol groups [131]. The surface charge density results from an equilibrium between counterions at the silica surface and free ions in the bulk electrolyte. 
By adding salt ions, the electrostatic interaction between the surface sites will be screened to accommodate more surface charges. Thus the surface charge density will be affected by the salt concentration.

The self-assembly process was begun with the cationic layer (PAH or PEI). The substrates were alternately immersed in PAH (or PEI) and PSS for 5 min for the first two bilayers and the time specified in Table 4.2 for the remaining bilayers. The substrates were rinsed in ultrapure water after the assembly of each layer. A total of 20 bilayers were assembled onto the substrates. Then samples were dried in nitrogen and measured immediately.

In order to investigate the thickness and refractive index of the polyelectrolyte self-assembled thin film, films on silicon substrates were measured by a variable angle spectroscopic ellipsometer (VASE, J. A. Woollam). Each of the samples was measured three times at different locations across the surface. A simplified Cauchy model was used to describe the refractive index

$$
n(\lambda)=A+\frac{B}{\lambda^{2}},
$$

where $\mathrm{A}$ and $\mathrm{B}$ are the fitted parameters.

\subsubsection{Results and analysis}

The results were analyzed in MINITAB (Minitab Inc). The effect of the factors on thickness and refractive index differed so the analysis includes two parts: analysis of thickness and analysis of refractive index.

Quality characteristic (QC) indicates the direction of desirability of the evaluation numbers. Depending on the criteria and how it is measured, QC can be selected from 'Bigger is better', 'Smaller is better' and 'Nominal is the best'. 'Nominal is the best' is selected when a target or nominal value is desired. In order to control the thickness or refractive index of the polymer self-assembly to a desired value, 'Nominal is the best' was chosen in our study.

A main effect describes the changes of the mean response across the levels of a factor. The relative strength of the effects across factors can be compared from the plots. 
Figure 4.2 and Figure 4.3 are the main effects plots for the thickness analysis. Figure 4.2 illustrates the main effects of factors for $\mathrm{S} / \mathrm{N}$ ratios and Figure 4.3 shows the main effects of factors for mean values. The relative effects of factors can be estimated from the figures. For example, the salt concentration and polymer species have much higher effects both on the $\mathrm{S} / \mathrm{N}$ ratios and on the means of thickness.

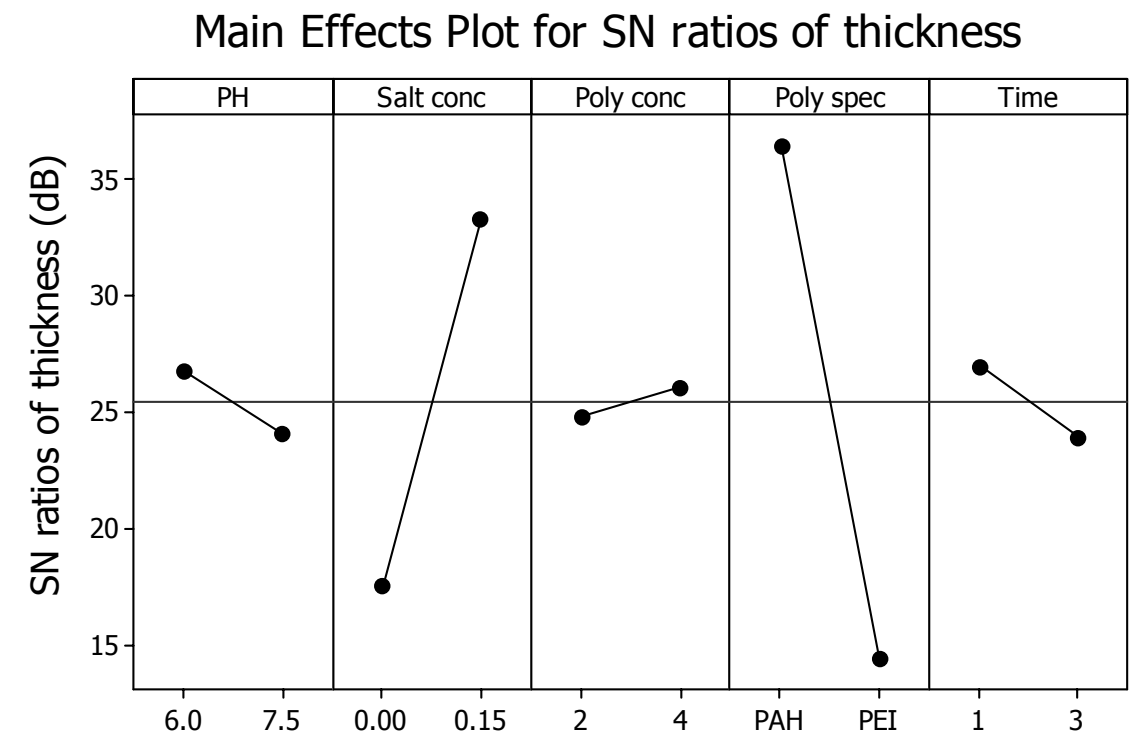

Figure 4.2 Main effects plot for $\mathrm{S} / \mathrm{N}$ ratios of thickness. 


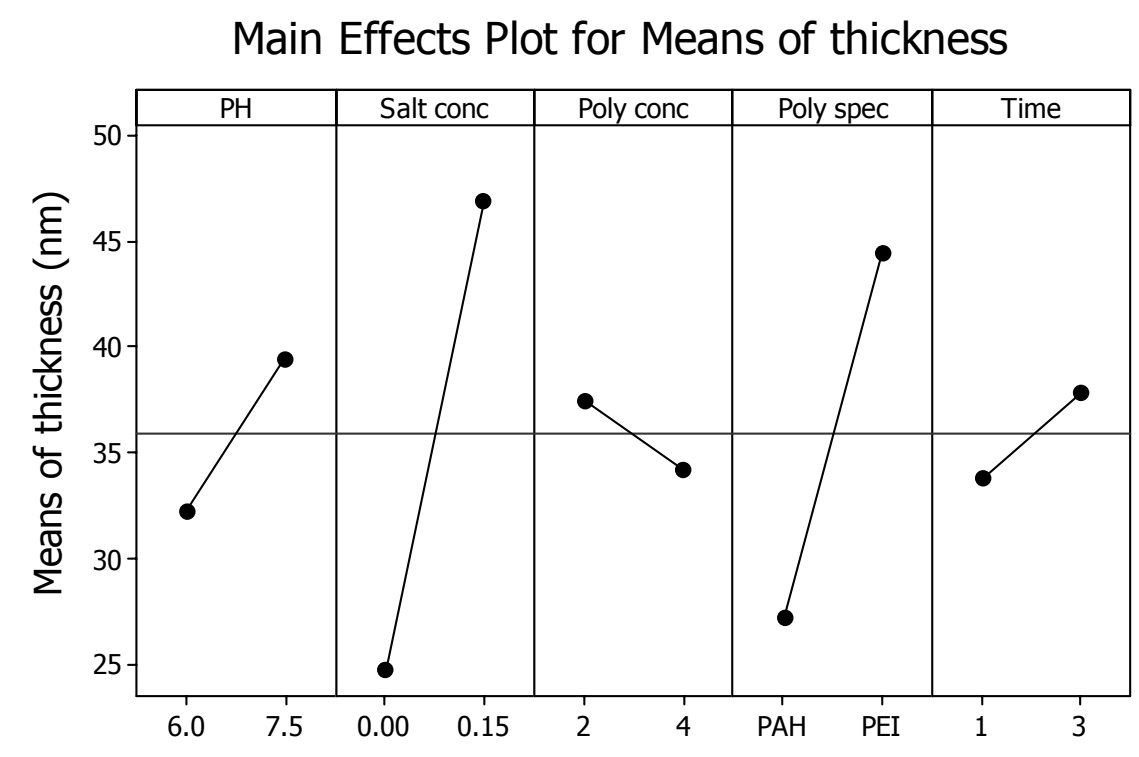

Figure 4.3 Main effects plot for means of thickness.

The relative effect of factors can be examined more accurately from the response tables. The response tables list the average of each response characteristic $(\mathrm{S} / \mathrm{N}$ ratios, means) for each level of each factor. The tables include ranks based on Delta statistics, which compare the relative magnitude of effects. The Delta statistic is the highest minus the lowest average for each factor. The ranks are assigned based on Delta values; rank 1 to the highest Delta value, rank 2 to the second highest, and so on. It is possible to determine which level of each factor provides the best result from level averages in the response tables. Table 4.3 lists the response of factors for $\mathrm{S} / \mathrm{N}$ ratios and Table 4.4 is for the mean values. According to Table 4.3 and Table 4.4, the polymer species and salt concentration are the dominant factors for $\mathrm{S} / \mathrm{N}$ ratio and mean values of thickness. The different conformations from polycation species (PAH, PEI) will definitely affect the layer thickness. Compared with PAH/PSS film, PEI/PSS film has higher average thickness (more than $60 \%$ thicker) and lower $\mathrm{S} / \mathrm{N}$ ratio (more than $20 \mathrm{~dB}$ lower). It may come from the different structures of the polycation molecules. It was reported that the thickness of the polyelectrolyte films can drastically be increased by addition of electrolyte in the polymer solutions [178]. The conformation of the polyelectrolyte 
changes with respect to the ionic strength. The molecular confirmation will take a more "loopy" form instead of "flat" form in the solution of higher ionic concentration. This can be explained by the screening of the electrostatic repulsion of the ionic groups on the chains of the polyelectrolyte.

Table 4.3 Response table for signal to noise ratios (in $\mathrm{dB}$ ) of thickness.

\begin{tabular}{|c|c|c|c|c|c|}
\hline Level & $\mathrm{pH}$ & $\begin{array}{c}\text { Salt } \\
\text { concentration }\end{array}$ & $\begin{array}{c}\text { Polymer } \\
\text { concentration }\end{array}$ & $\begin{array}{c}\text { Polymer } \\
\text { species }\end{array}$ & Time \\
\hline 1 & 26.76 & 17.54 & 24.82 & 36.44 & 26.97 \\
\hline 2 & 24.11 & 33.33 & 26.05 & 14.43 & 23.90 \\
\hline Delta & 2.65 & 15.78 & 1.22 & 22.01 & 3.06 \\
\hline Rank & 4 & 2 & 5 & 1 & 3 \\
\hline
\end{tabular}

Table 4.4 Response table for means of thickness (in nm).

\begin{tabular}{|c|c|c|c|c|c|}
\hline Level & $\mathrm{pH}$ & $\begin{array}{c}\text { Salt } \\
\text { concentration }\end{array}$ & $\begin{array}{c}\text { Polymer } \\
\text { concentration }\end{array}$ & $\begin{array}{c}\text { Polymer } \\
\text { species }\end{array}$ & Time \\
\hline 1 & 32.29 & 24.84 & 37.53 & 27.27 & 33.86 \\
\hline 2 & 39.50 & 46.95 & 34.26 & 44.52 & 37.93 \\
\hline Delta & 7.20 & 22.12 & 3.26 & 17.25 & 4.08 \\
\hline Rank & 3 & 1 & 5 & 2 & 4 \\
\hline
\end{tabular}

Similar to the analysis for the thickness of polymer self-assembly, the main effects plots (Figure 4.4, Figure 4.5) and response tables (Table 4.5, Table 4.6) for refractive index are also derived from the experimental results. Figure 4.4 and Figure 4.5 are the main effects plots for the $\mathrm{S} / \mathrm{N}$ ratios and mean values, respectively. From the figures, polymer species and salt concentrations are the dominant factors for $\mathrm{S} / \mathrm{N}$ ratios but it is difficult to define the dominant factors for mean values of refractive index. Most of the factors have similar effect on the mean values. According to the results in Table 4.6, the variations of response between factors and levels are small. Comparison of the 
mean values in Table 4.4 and Table 4.6 shows that the factors have less effect on the refractive index than on the thickness. Factors can change the thickness of the selfassembly by affecting the molecular conformation. However, the refractive index of the self-assembly is dependent on the structure and component of the bulk materials. The self-assembly is a multilayer buildup of polycation and polyanion molecules. The closepacked nature of the self-assembly is not highly affected by the molecular conformation, so the refractive index is not closely related with the factors and levels that affect the conformation. According to the results, even change of the polycation from PAH to PEI does not vary the refractive index a lot.

Main Effects Plot for SN ratios of refractive index

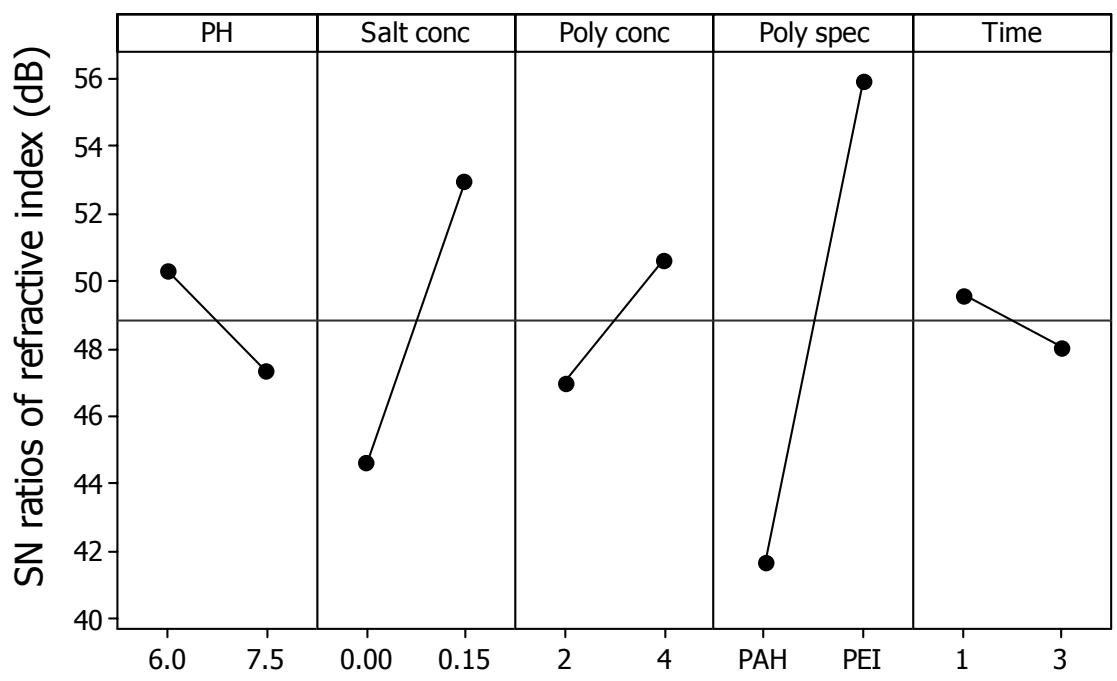

Figure 4.4 Main effects plot for $\mathrm{S} / \mathrm{N}$ ratios of refractive index. 


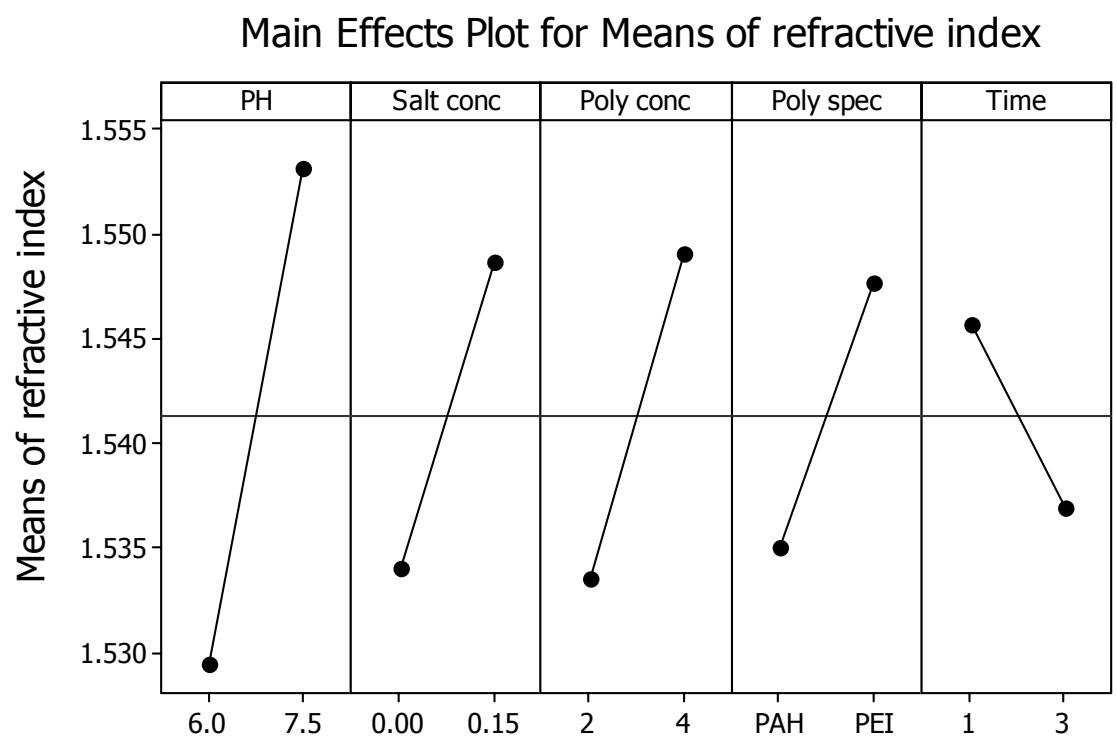

Figure 4.5 Main effects plot for means of refractive index.

Table 4.5 Response table for signal to noise ratios (in $\mathrm{dB}$ ) of refractive index.

\begin{tabular}{|c|c|c|c|c|c|}
\hline Level & $\mathrm{pH}$ & $\begin{array}{c}\text { Salt } \\
\text { concentration }\end{array}$ & $\begin{array}{c}\text { Polymer } \\
\text { concentration }\end{array}$ & $\begin{array}{c}\text { Polymer } \\
\text { species }\end{array}$ & Time \\
\hline 1 & 50.30 & 44.66 & 47.02 & 41.67 & 49.59 \\
\hline 2 & 47.33 & 52.98 & 50.61 & 55.96 & 48.04 \\
\hline Delta & 2.96 & 8.32 & 3.59 & 14.29 & 1.55 \\
\hline Rank & 4 & 2 & 3 & 1 & 5 \\
\hline
\end{tabular}

Table 4.6 Response table for means of refractive index.

\begin{tabular}{|c|c|c|c|c|c|}
\hline Level & $\mathrm{pH}$ & $\begin{array}{c}\text { Salt } \\
\text { concentration }\end{array}$ & $\begin{array}{c}\text { Polymer } \\
\text { concentration }\end{array}$ & $\begin{array}{c}\text { Polymer } \\
\text { species }\end{array}$ & Time \\
\hline 1 & 1.529 & 1.534 & 1.534 & 1.535 & 1.546 \\
\hline 2 & 1.553 & 1.549 & 1.549 & 1.548 & 1.537 \\
\hline Delta & 0.024 & 0.015 & 0.016 & 0.013 & 0.009 \\
\hline Rank & 1 & 3 & 2 & 4 & 5 \\
\hline
\end{tabular}


The relative variation of refractive index (Table 4.6) is much smaller than that of thickness (Table 4.4) in the examined factors and levels. So the factors of S/N ratio in thickness have higher priority than that in refractive index. From Table 4.3, polymer species and salt concentration are the dominant factors of $\mathrm{S} / \mathrm{N}$ ratio in thickness. $\mathrm{PAH}$ in polymer species and $0.15 \mathrm{M}(\mathrm{NaCl})$ in salt concentration are selected for future experiments since those levels corresponding to higher $\mathrm{S} / \mathrm{N}$ ratios. Other factors such as $\mathrm{pH}$, polymer concentration and dipping time can be selected according to the requirement of the experiment.

\subsubsection{Stability of the polymer self-assembly}

Long term stability of the polymer self-assembly was examined. A thin film with structure $[\mathrm{PAH} / \mathrm{PSS}]_{10}$ was fabricated and kept at room temperature for more than a month. Figure 4.6 shows the variations of thickness and refractive index $(\lambda=589 \mathrm{~nm})$ of the film. The thickness has a relative change of $\pm 0.4 \%$ which is about $\pm 0.1 \mathrm{~nm}$ in more than 30 days. At the same time, there is a $\pm 0.6 \%$ change in the refractive index. These variations in thickness and refractive index are most likely caused by the release of bound water molecules from the film and reentry from the air due to temperature and humidity changes [24]. The slight variation will not affect subsequent immobilization of biological molecules. 


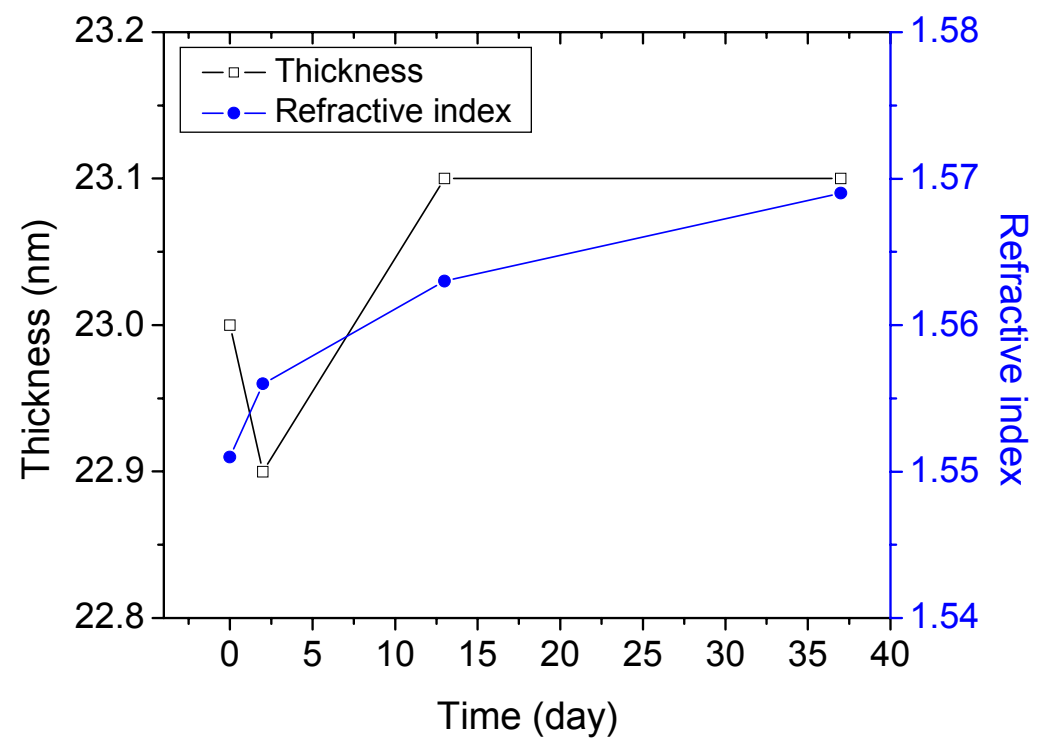

Figure 4.6 Variations in thickness and refractive index of [PAH/PSS $]_{10}$ film at room temperature.

\subsubsection{Immobilization of IgG and immunosensing}

A silicon substrate was first cleaned in piranha solution for $1 \mathrm{~h}$, after which the substrate was ultrasonically rinsed in ultrapure water and dried under nitrogen. PAH (2 $\mathrm{mg} / \mathrm{ml})$ and PSS ( $2 \mathrm{mg} / \mathrm{ml})$ solutions were prepared using ultrapure water. $\mathrm{pH}$ values of the solution were adjusted to 7.5 (Corning 455 Ion Analyzer). Films were prepared by alternately dipping the wafer in the appropriately charged polyelectrolyte solution. PAH or PSS layers were deposited by immersing for $3 \mathrm{~min}$ ( $5 \mathrm{~min}$ for the first two bilayers) in the polyelectrolyte solution, followed by rinsing in ultrapure water and drying. A film with structure $[\mathrm{PAH} / \mathrm{PSS}]_{20} \mathrm{PAH}$ was fabricated and measured by an ellipsometer (VASE, J. A. Woollam). The film had a thickness of $15.18 \pm 0.04 \mathrm{~nm}$ and refractive index of $1.561 \pm 0.007(\lambda=589 \mathrm{~nm})$. Then the wafer was placed in a flow cell mounted on the ellipsometer for monitoring immobilization of rabbit IgG and immunoreactions. The wafer was immersed in the rabbit IgG solution ( $25 \mu \mathrm{g} / \mathrm{ml}$ HEPES buffer) for $1 \mathrm{~h}$. The dynamic changes in the film thickness are shown in Figure 4.7. After rinsing in pure 
water, the thickness of the IgG layer was measured to be $3.6 \mathrm{~nm}(\mathrm{n}=1.45)$. When the wafer was immersed in HEPES buffer, no obvious desorption of IgG was observed. The immunoreactions between the immobilized rabbit IgG and anti-rabbit IgG solution ( 25 $\mu \mathrm{g} / \mathrm{ml}$ HEPES buffer) can be observed in Figure 4.7. The anti-IgG layer was measured to be $6.1 \mathrm{~nm}(\mathrm{n}=1.45)$ after rinsing.

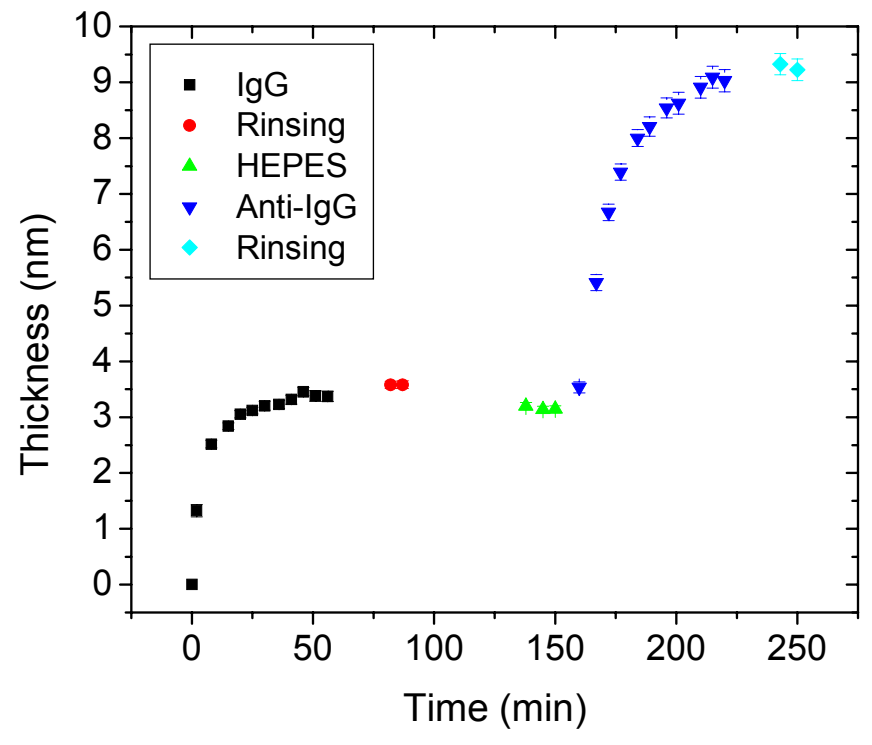

Figure 4.7 IgG immobilization on polymer film and immunosensing.

This experiment demonstrates that polymer self-assembly as a surface modification technique can effectively adsorb biomolecules and retain their biological activity. Therefore we applied this technique on our fiber optic sensors for immobilization of receptors and further immunosensing.

\subsection{Thin film characterization by spectroscopy}

The multilayer buildup can also be investigated by UV/VIS spectroscopy. The optical absorbance increases along with the deposition. The absorption of the multilayer film is due to both the cationic and anionic polymers. Here we first examined the 
absorption of PAH and PSS separately by UV-VIS spectroscopy (UV-2501 Shimadzu). From Figure 4.8 there is no apparent peak in the PAH absorption spectrum in the UVVIS (200-900 nm) range. For PSS, the absorption peak $(225 \mathrm{~nm})$ originates from the aromatic chromophore of the styrene monomer unit (Figure 4.9) [179].

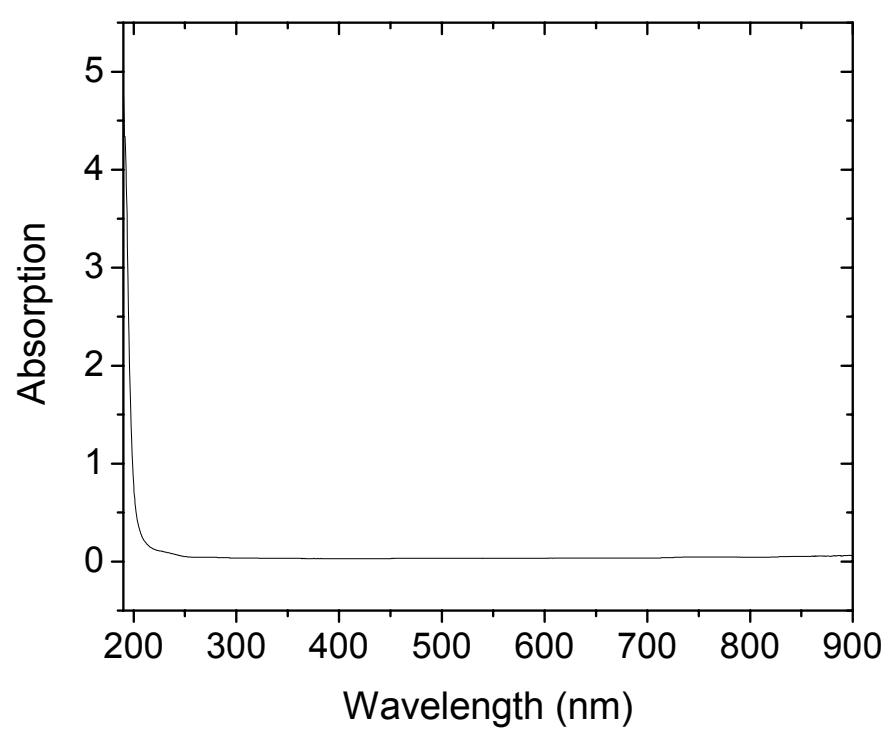

Figure 4.8 UV/VIS absorption spectrum of PAH solution $(0.4 \mathrm{mg} / \mathrm{ml})$. 


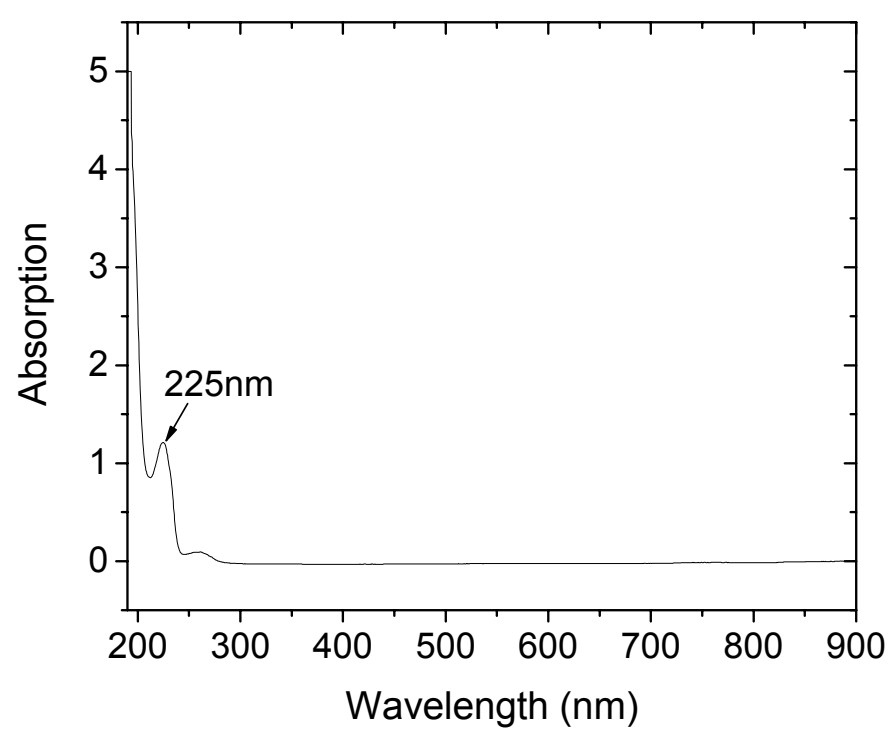

Figure 4.9 UV/VIS absorption spectrum of PSS solution $(0.04 \mathrm{mg} / \mathrm{ml})$.

A film with structure of $[\mathrm{PAH} / \mathrm{PSS}]_{\mathrm{n}}$ was self-assembled onto a silica wafer. During preparation of the PAH/PSS multilayer, absorption increased regularly with the number of layers (Figure 4.10). Both the $195 \mathrm{~nm}$ and $225 \mathrm{~nm}$ bands are the response of the PSS layer numbers. Figure 4.11 shows the absorbance of PSS at $225 \mathrm{~nm}$ with respect to the bilayer number. The fitting shows that the absorbance increases linearly with the layer numbers. The absorbance increase in the first several bilayers is smaller because of the thinner film in initial layers, which is similar to the results from ellipsometry and other methods. 


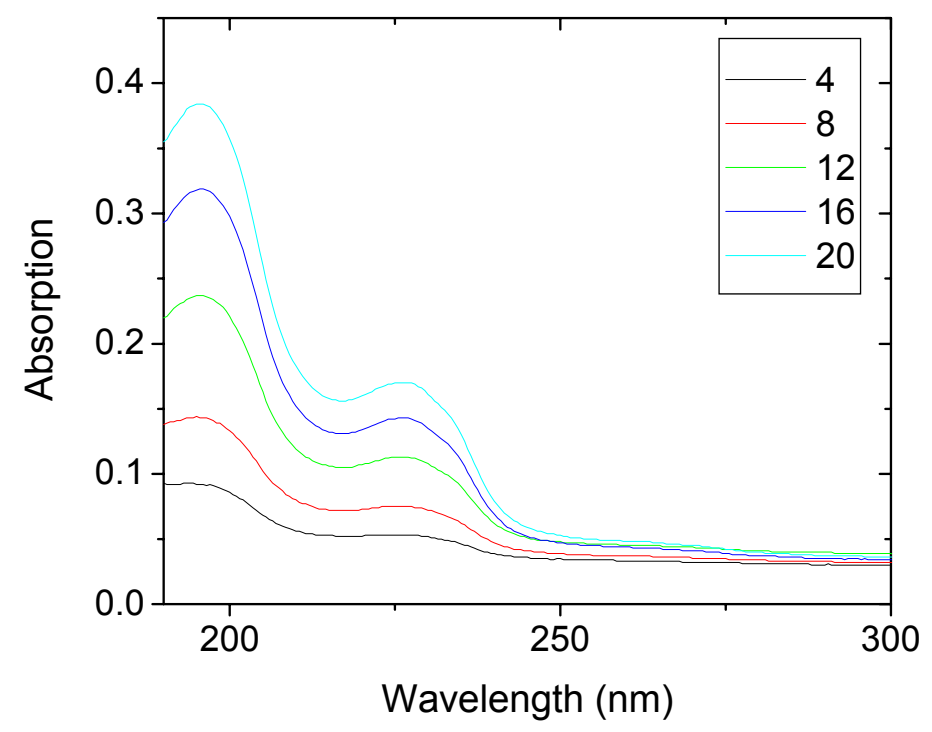

Figure 4.10 UV/VIS absorption spectra of PAH/PSS multilayer film during the fabrication.

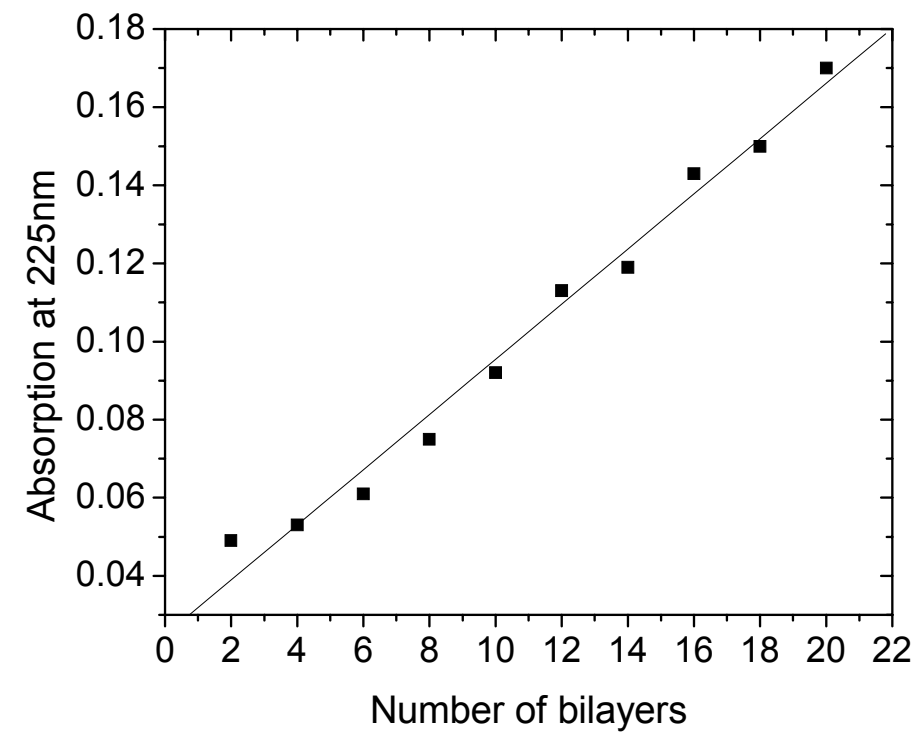

Figure 4.11 The absorbance of the PSS at $225 \mathrm{~nm}$ versus the bilayer number. 
Proteins can be incorporated into the polymer self-assembly [175, 185]. The embedded proteins can keep their biological activity with respect to immunological reactions even when thin polyelectrolyte layers were deposited over them [175]. Bovine serum albumin (BSA) was selected to examine the construction of multilayer proteincontaining films. A self-assembled film with structure of $[\mathrm{PAH} / \mathrm{BSA}]_{7}$ was coated on a silica substrate. Figure 4.12 compares the absorption spectra of the substrate before and after the self-assembling process. The absorption peak at $279 \mathrm{~nm}$ is from the BSA molecules. This effect provides the potential to design bioactive materials with different biological responses.

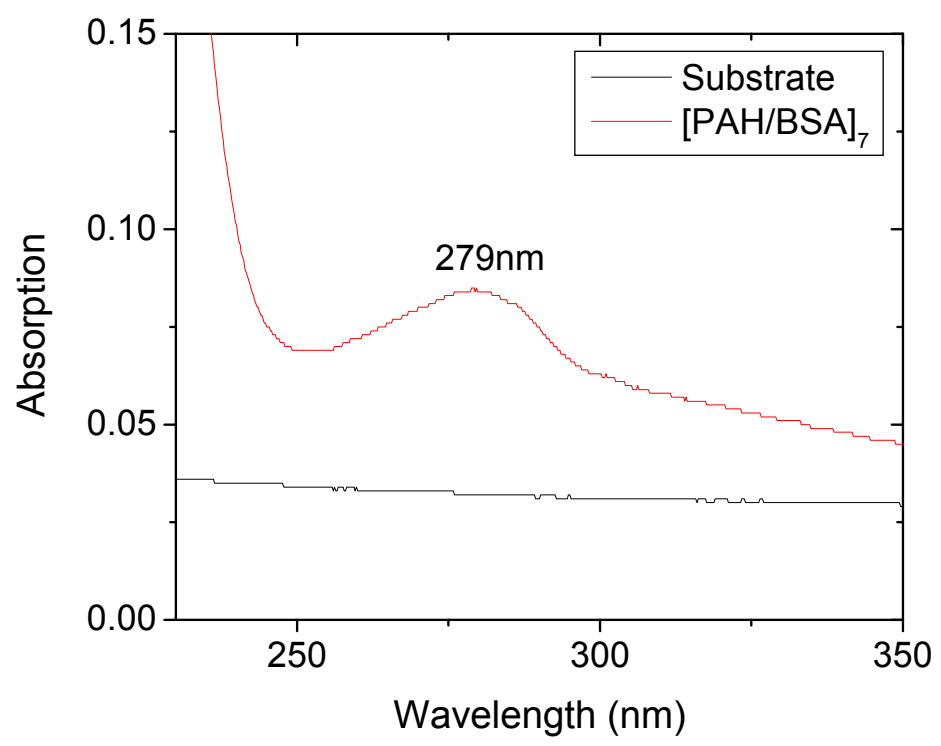

Figure 4.12 Comparison of absorption spectra of substrate with and without PAH/BSA film.

In multicavity sensor applications, a PAH/PSS film is deposited onto the sensor end. The thin film works as a precursor film for immobilization of biological molecules. The absorbance characteristics in the detection wavelength range $(1520-1570 \mathrm{~nm})$ are important in the analysis of the sensor output signal. UV-VIS-NIR spectrum (UV-3101 
Shimadzu, Figure 4.13) of PAH/PSS films confirms that the film absorption is mostly in the UV range. The absorption in the detection wavelength range can be neglected in the analysis.

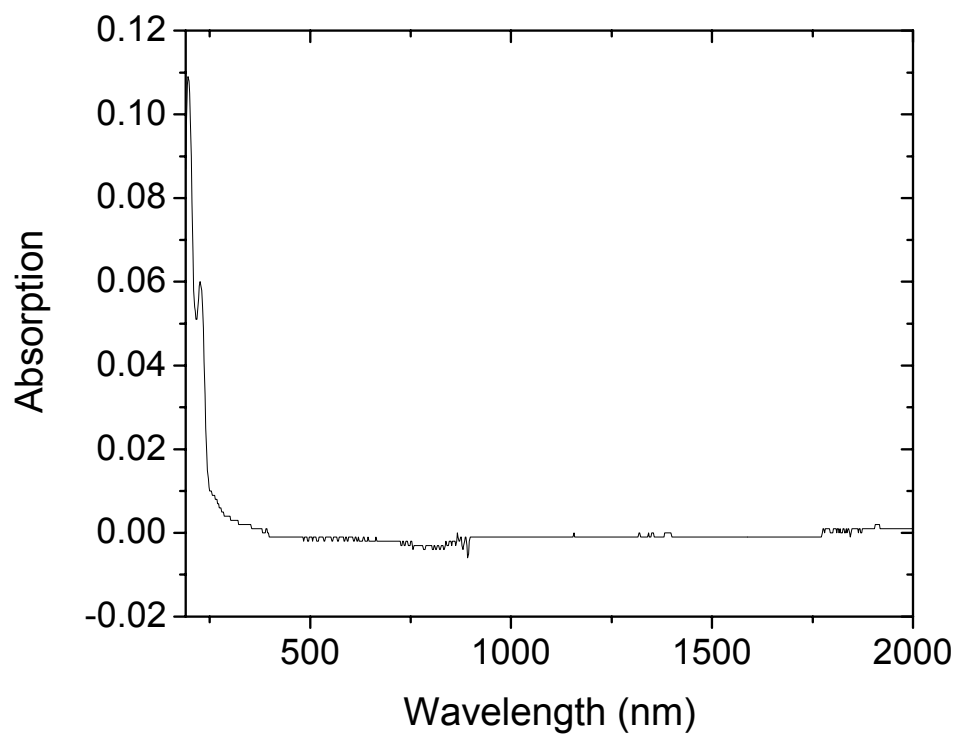

Figure 4.13 UV-VIS-NIR absorption spectrum of PAH/PSS multilayer film.

\subsection{Polyelectrolyte self-assembly on fiber and sensor}

The electrostatic self-assembly process has been applied to deposit nanofilms on various substrates. FP cavities [105] or gratings [194] can be formed on the fiber end by direct deposition of thin film. The composition of the nanofilm is selected according to the sensing application. A fiber optic humidity sensor was designed based on FP interferometry since the reflection coefficient of the external mirror changes due to its hygroscopic nature [106]. The reflection spectrum of the FP cavity is also sensitive to the absorbance of cavity materials, which can be applied in chemical sensing [107]. The measurement of coating thickness is based on the observation of the oscillations in reflected optical power due to the periodic interferometric nature of the coating at the 
fiber end. Since each layer of self-assembled thin film is only a few nanometers, tens or even hundreds of layers are needed to be able to observe the oscillations. Furthermore, the thickness resolution is limited by the intensity-based measurement. High-resolution direct measurement of nanofilms was achieved by multicavity FP sensors, overcoming these difficulties. The adsorption of the self-assembly to the endface of the FP cavity will shift the reflection spectrum. Measurement of polymer self-assembly as thin as a single monolayer is possible due to the high sensitivity of FP sensors.

\subsubsection{Self-assembly on fiber end}

In developing a fiber-optic biosensor, it is important to determine the differences between films on wafers and fibers. It was reported that some polyelectrolyte selfassembled films have similar characteristics on silicon wafers and optical fibers [105]. However, our results indicate that self-assembled films on fiber may have a greater average thickness than that on wafers while the refractive index is similar.

In order to compare the thin film characteristics on wafer and fiber, a polymer thin film was self-assembled on the fiber end to form a FP cavity. The refractive index and thickness of the film on fiber end can be obtained from the FP cavity reflection. The experimental setup is shown in Figure 4.14. An LED with wavelength $\lambda=1.3 \mu \mathrm{m}$ was the light source. The optical spectrum analyzer (OSA, Ando AQ-6315A) measured the reflection spectrum from the FP cavity on the cleaved fiber end. The other end of the coupler is covered with index matching gel to minimize interaction from undesired reflections. 


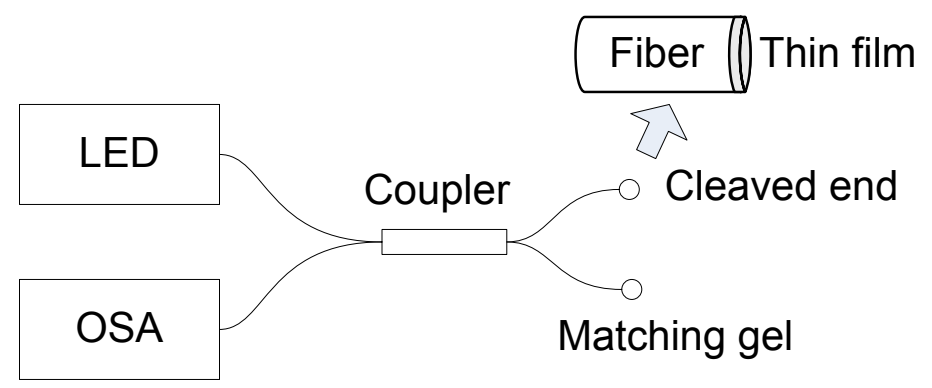

Figure 4.14 Experimental setup for thin film FP cavity.

The reflection intensity of the FP cavity is [105]

$$
R_{F P}=\frac{R_{1}+R_{2}\left(1-A_{1}\right)^{2} \exp (-4 \alpha d)+2 \sqrt{R_{1} R_{2}}\left(1-A_{1}\right) \exp (-2 \alpha d) \cos \phi}{1+R_{1} R_{2} \exp (-4 \alpha d)+2 \sqrt{R_{1} R_{2}} \exp (-2 \alpha d) \cos \phi},
$$

where $R_{1}$ and $R_{2}$ are the reflection coefficients of each surface of the FP cavity. $A_{1}$ is the excess loss of the first reflection surface. $\alpha$ is the absorption coefficient of the film.

$$
\phi=4 \pi n d / \lambda+\pi
$$

is the phase shift of the FP cavity. $n$ and $d$ are the refractive index and thickness of the film respectively. When the light was reflected from a medium with higher refractive index, a $\pi$ phase shift was added to the total phase shift. The reflection intensity changes as the thin film is gradually assembled onto the fiber end. Then the thickness and refractive index of the film can be calculated from Eq. 4.2 and 4.3.

Polydiallyldimethylammonium chloride (PDDA, Aldrich) as the cationic electrolyte and a polymer dye (Poly S-119, Aldrich) as the anionic electrolyte were chosen for the self-assembly process. A bilayer structure of the form [PDDA/PS119] was built up on the cleaved fiber end where $n$ indicates the bilayer number. The interferometric nature of the thin film cavity can be observed in Figure 4.15. The period of the experiment data in Figure 4.15 is around 49 bilayers. The refractive index of the film can be fitted from the experimental results as 1.59. Then the average thickness of a bilayer can be obtained from Eq. 4.3 as $8.3 \mathrm{~nm}$. 


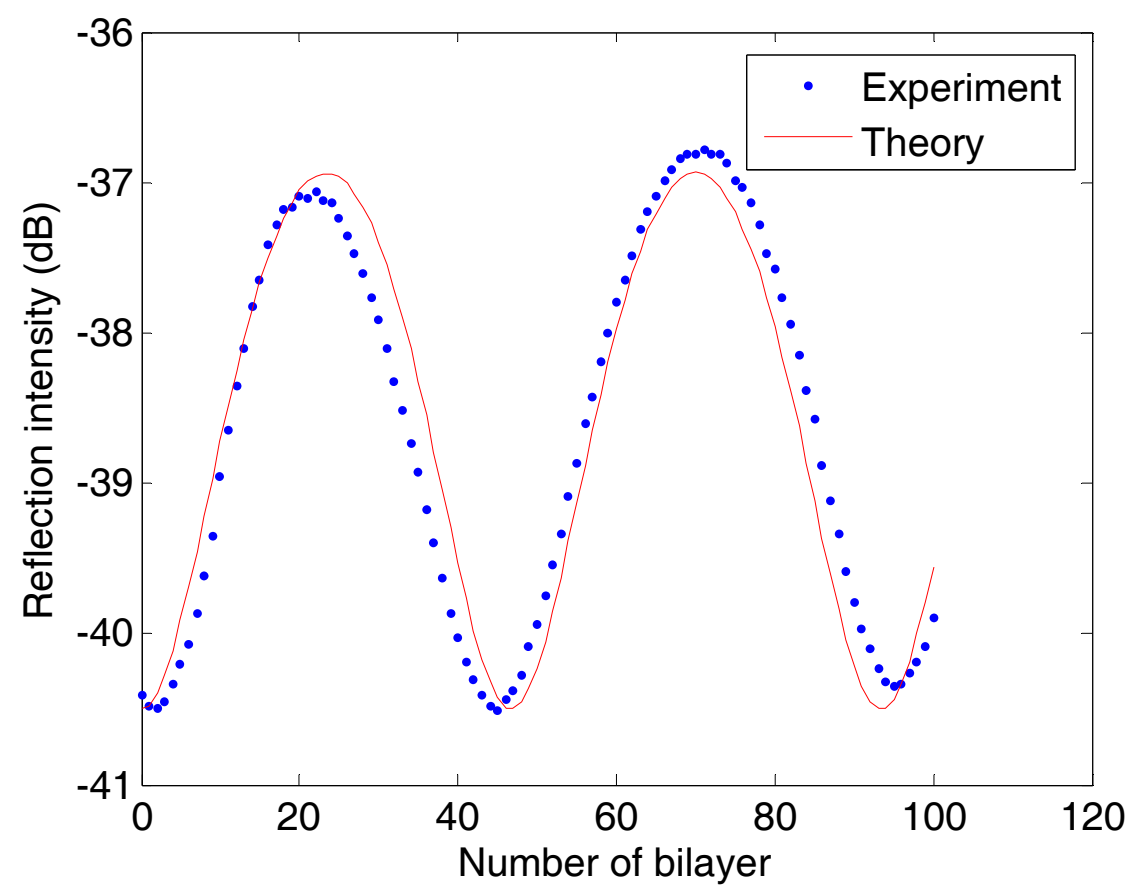

Figure 4.15 The reflection intensity changes with respect to the number of bilayers of PDDA/PS119.

[PDDA/PS119 $]_{\mathrm{n}}$ film was coated on the silicon substrate using identical conditions. The thickness and refractive index of the coating were measured with an ellipsometer (VASE, J. A. Woollam). The average thickness of a bilayer is about $4.2 \mathrm{~nm}$ and refractive index is around 1.59. The average thickness of a PDDA/PS119 bilayer on fiber is almost 2 times of that on wafer, while the refractive index is similar.

A similar test was implemented with [PAH/PSS $]_{\mathrm{n}}$ film. As shown in Figure 4.16 (a), the thickness and refractive index of the film on fiber end was obtained as 9.6 $\mathrm{nm} /$ bilayer and 1.53. The corresponding thickness and refractive index of the film on silicon wafer were $2.6 \mathrm{~nm} /$ bilayer and 1.54 by ellipsometry. The average thickness of a $\mathrm{PAH} / \mathrm{PSS}$ bilayer on fiber end is more than 3 times of that on wafer while the refractive index is similar. 


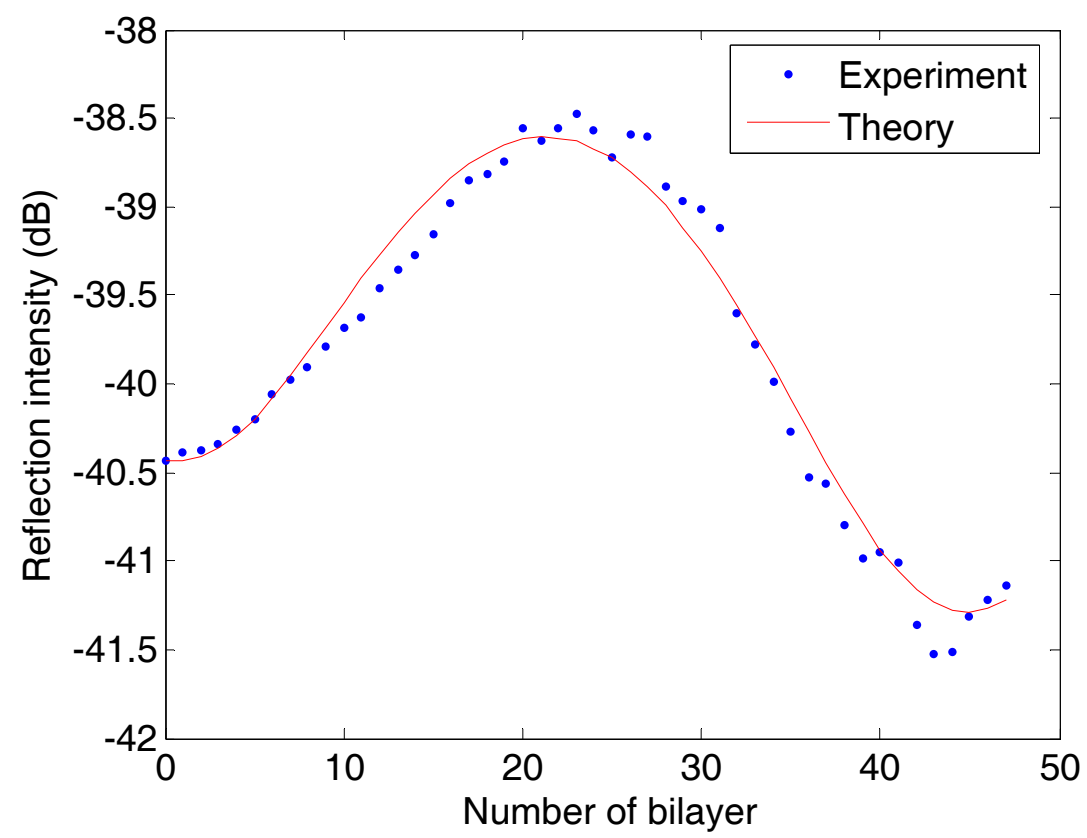

(a)

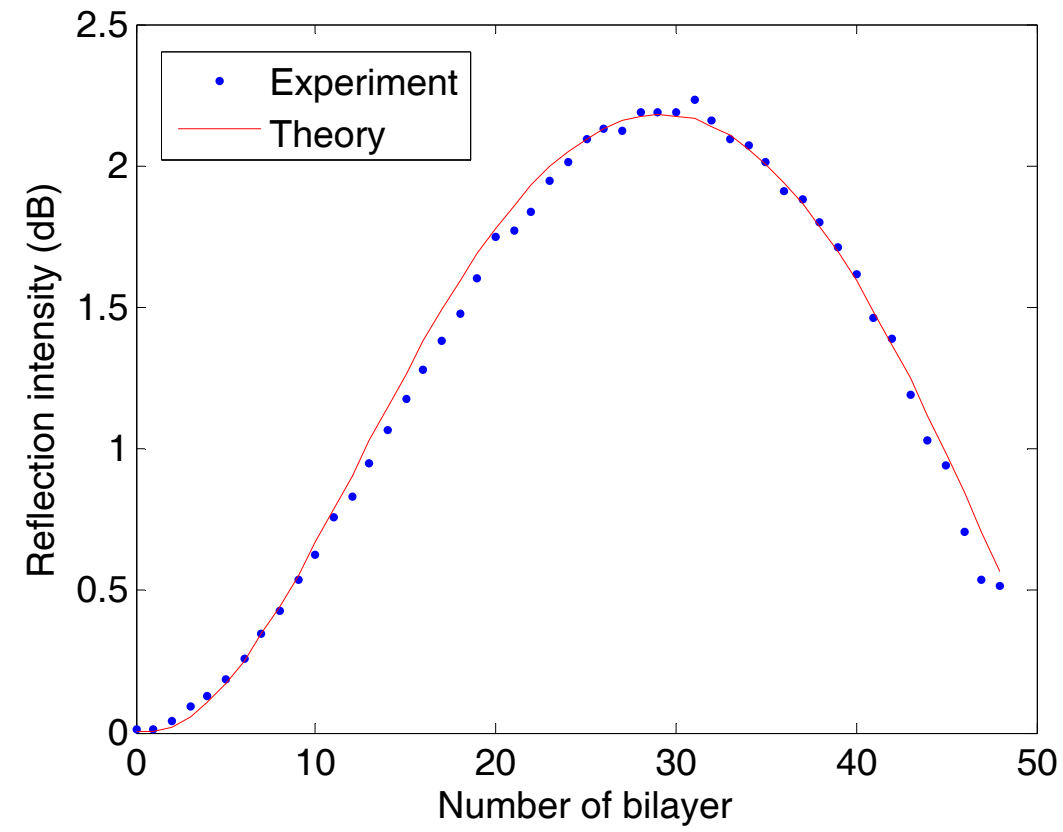

(b)

Figure 4.16 The reflection intensity changes with respect to the number of bilayers of PAH/PSS. (a) $\lambda=1.3 \mu \mathrm{m}$;(b) $\lambda=1.52 \mu \mathrm{m}$. 
Similar experiment on $[\mathrm{PAH} / \mathrm{PSS}]_{\mathrm{n}}$ film was done by replacing the LED and OSA with the CTS using wavelength $\lambda=1.52 \mu \mathrm{m}$ (Figure 4.16 (b)). The average thickness was $8.3 \mathrm{~nm} /$ bilayers and refractive index was 1.54 . The difference in the thickness per layer may be due to different rinsing techniques. Manual rinsing and ultrasonic rinsing were used in the OSA and CTS tests respectively. Compared to manual rinsing, ultrasonic rinsing has better repeatability on average thickness. Therefore we chose ultrasonic rinsing for further polymer self-assembly experiments.

The difference in average thickness between the films on fiber and wafer indicates that the molecular interactions and conformations may be affected by the substrate. The polymer chains may entangle with each other. The molecules seem to entangle more easily with others on fiber end than on the silicon wafer. The thickness difference is also related to the molecular conformation, so the difference may vary with polymer species. Further investigation on the interactions between the polymers and different substrates is needed to reveal the details.

\subsubsection{Self-assembly on multicavity sensors}

In Chapter 2 and 3, two kinds of multicavity FP sensors have been reported for thin-film measurement. The mechanism of self-assembling of polymer films on the multicavity sensor is the same as that on the fiber end. However, compared to the intensity-based detection method described in the last section, the interferometric method has better resolution and is immune to the intensity fluctuations of the light source due to temperature and other factors.

Both the tubing-based sensor and microgap sensor were applied for measurement of polymer self-assembly. Sensors were first cleaned in a piranha solution for $15 \mathrm{~min}$, after which sensors were ultrasonically rinsed in ultrapure water. The sensor surface is negatively charged in the polymer solutions. Films were prepared by alternately dipping the sensor in the appropriately charged polyelectrolyte solution. PAH or PSS layers were deposited alternately by immersion for $5 \mathrm{~min}$ in the polyelectrolyte solution, followed by rinsing in ultrapure water and drying in air. Reflection spectra were recorded to monitor 
the film growth. The thin-film sensing is based on the measurement of optical thickness changes of the fiber-film cavity caused by the adsorption of target molecules. The adsorbed thin film can be estimated as an extension of the fiber cavity when the refractive index of the film is similar to the fiber. High sensitivity can be achieved due to the intrinsic nature of Fabry-Perot interferometry. Figure 4.17 shows the changes in optical thickness of polymer self-assembly [PAH/PSS $]_{\mathrm{n}}$ measured by tubing-based FP sensor. The average thickness per bilayer is $8.6 \pm 0.5 \mathrm{~nm}(n=1.54)$. The self-assembly process was also examined by microgap FP sensors. Figure 4.18 shows the optical thickness measurement by a microgap sensor. The film thickness is $8.2 \pm 0.3 \mathrm{~nm}$ per bilayer $(n=1.54)$. The average thickness of each bilayer is similar to the intensity-based measurement. Furthermore, the interferometric method has better resolution for tracing even the change by a single molecular layer. After the first two bilayers, the film thickness tends to increase linearly with the bilayer number. However, the contributions from PAH and PSS layer are not the same. The average thickness of a PAH layer $(3.2 \pm 0.5 \mathrm{~nm})$ is thinner than that of a PSS layer $(5.8 \pm 0.5 \mathrm{~nm})$. This is not an unexpected result that while the bilayer thickness should remain the same, the thickness contributions of different molecules (e.g. the anion and the cation) are not expected to be the same. The dimensions or the deformations of the coils from polymer-solvent interactions may have a major effect. Another possible reason is that the side-group of PSS is bigger than that of PAH [195]. 


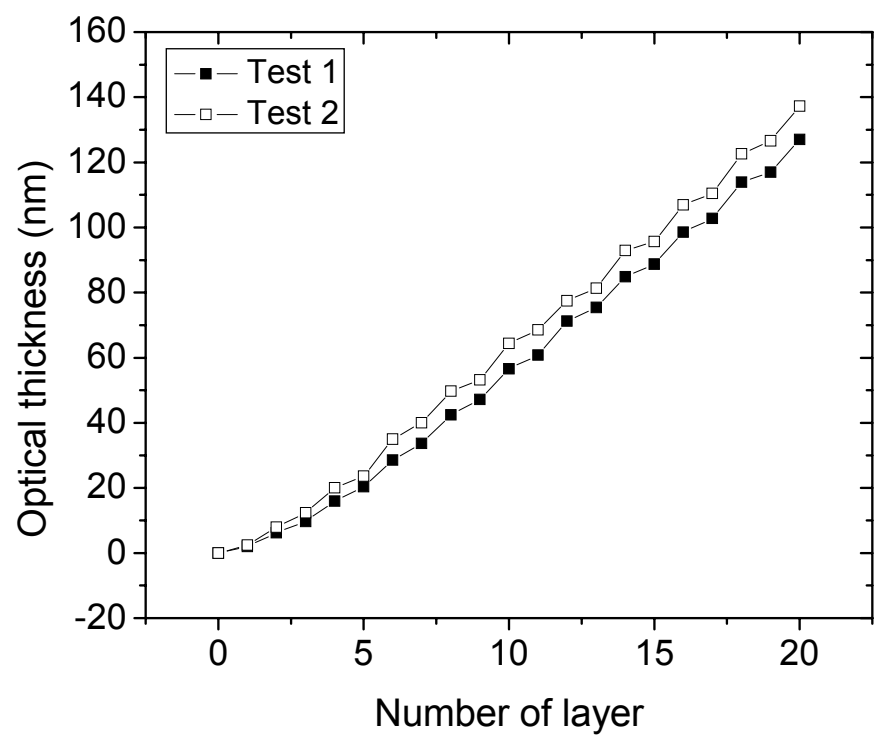

Figure 4.17 Optical thickness changes with the number of PAH/PSS layers on a tubingbased FP sensor.

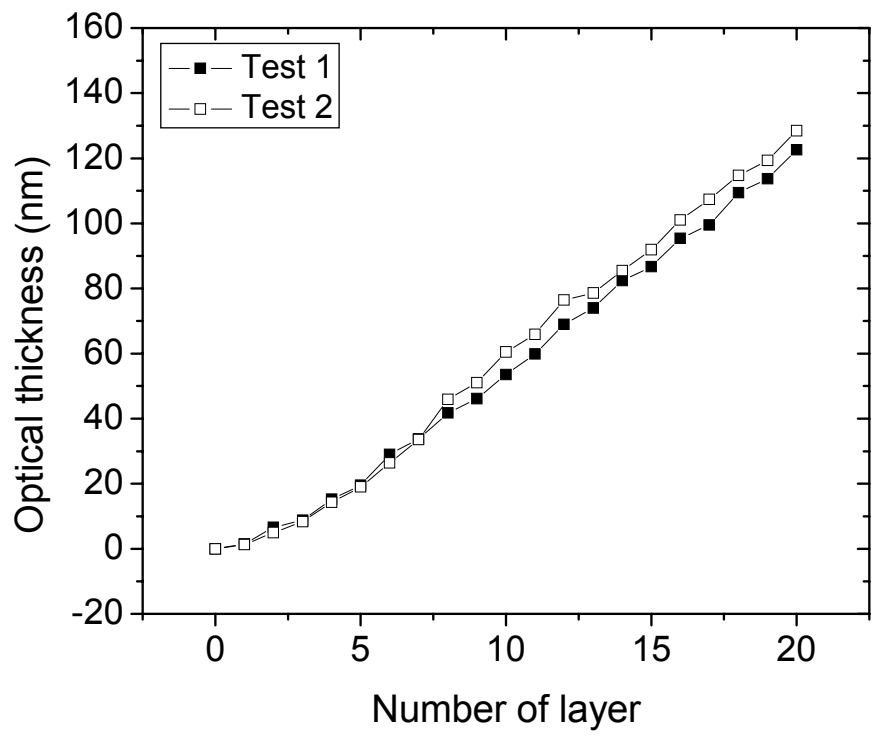

Figure 4.18 Optical thickness changes with the number of PAH/PSS layers on a microgap FP sensor. 


\subsection{Conclusions}

In this chapter, the characteristics of polymer self-assembly were investigated for surface modification and immobilization of biological molecules in fiber optic biosensing. The multilayer buildup on wafers was examined by ellipsometry and UV-VIS-NIR spectroscopy. From the evaluation of the ellipsometry results we obtained the effects of multiple factors on thickness and refractive index. Experimental design and analysis were applied to determine the relative effects of the factors. Polymer species and salt concentration are the dominant factors of $\mathrm{S} / \mathrm{N}$ ratio both in thickness and refractive index. Systematic study of the multiple factors provides the optimum conditions in the fabrication of the precursor film for biosensing. Slight variations in the thickness and refractive index of polymer self-assembly were observed during long-term storage, which is most likely caused by the release of bound water molecules from the film and reentry from the air due to temperature and humidity changes. This minor effect will not affect further immobilization of biomolecules. It has been demonstrated that polymer selfassembly as a surface modification technique can effectively adsorb biomolecules and retain their activities. The growth of the self-assembled film was also monitored by the linear increase in absorbance with the number of layers by UV-VIS spectroscopy. The negligible absorbance of the PAH/PSS film in the NIR range made it suitable as a biosensing precursor film without significant loss in the reflection signals. Multilayer of

proteins can be incorporated into the self-assembly. This provides the potential to design bioactive materials with different biological responses.

Polymer thin-film self-assembled onto fibers was also investigated by intensitybased measurement. The difference in average thickness between the films on fiber and wafer indicates that the molecular interactions and conformations may be affected by the substrate. The interferometric method for thin-film measurement demonstrates higher sensitivity. Single monolayer adsorption can be monitored by multicavity FP sensors. The thickness difference existing between the components reveals more detailed information on the self-assembly process. The high sensitivity of multicavity sensors provides the possibility for sensing immunoreactions at the interfaces. 


\section{Chapter 5 Protein adsorption and immunosensing}

\subsection{Introduction}

In biosensing, it is essential that the biological recognition molecules be immobilized while retaining the activity to recognize the analyte. Biological receptors include proteins, nucleic acids, organelles and cells. The focus of this chapter is the surface modification techniques for protein immobilization and subsequent immunosensing. A number of different strategies have been used for immobilization of proteins $[11,22,111,196]$. The selection of procedures depends on the nature of the biological molecules, the composition of the adsorption surface and on the optical configuration. Immobilization methods can be classified as physical adsorption, covalent attachment, biomolecular interactions and host matrix encapsulation. Among those procedures, physical adsorption is the simplest method for immobilizing biomolecules onto the sensor surface. The protein-surface interactions are determined by the physical state of the surface and proteins and the solution environment. Electrostatic and hydrophobic interactions are the major driving forces in physical adsorption.

The silica surface of the optical fiber is able to absorb proteins. However, proteins are denatured (the hydrogen-bonded configuration is broken) by the forces that flatten the molecules against the surface [131]. Therefore, surface modification is necessary to retain activity of proteins in fiber optic biosensors. Electrostatic self-assembly (ESA) is an effective technique in surface modification. Proteins can adsorb onto the polymer film by hydrophobic and electrostatic interactions. The surface-charge polarity is selectable since positively and negatively charged surfaces appear alternately in self-assembled polyelectrolyte films. The electrostatic interaction may dominate the protein adsorption [197]. The adsorbed amounts are usually larger on an oppositely charged surface.

However, other factors may counteract the electrostatic interaction. It was observed that the proteins strongly interact with polyelectrolyte multilayers whether they have identical or opposite charges [198]. This can possibly be explained by the hydrophobic interaction between the protein and the polyelectrolyte multilayer, which is one of the key factors in 
protein adsorption. Proteins can also be incorporated into self-assembled multilayers, thus increasing the sensing capacity through the fabrication of a multilayer antibody film.

After immobilization of a receptor, the fiber optic sensor can be defined as a biosensor because of the biorecognition capability. Immunosensors, based on the interaction between immobilized immunoglobulin $\mathrm{G}(\mathrm{IgG})$ and their corresponding antigen, are one of the most developed biosensors. Hydrogen bonds are the major interactions of antibody-antigen complexes. Other interactions such as hydrophobic interactions, electrostatic interactions and van der Waals forces also increase the binding specificity between the antibody and antigen. Interactions existing in many locations over the molecules improve the binding affinity.

Various techniques have been applied in the study of protein adsorption and binding. The most commonly used techniques include optical techniques such as ellipsometry [32], Fourier transform infrared spectroscopy [199], fluorescence spectroscopy [200], reflectometry [201] and surface plasmon resonance (SPR) [202]. Other methods such as radiolabeling [203] and scanning force microscopy [204] also exist. Compared to those techniques, a fiber optic sensor has the advantage of miniature size thus allowing the use of a small sample volume. It can be used to monitor either the equilibrium or the kinetics of the interaction without the introduction of radioactive, fluorescent or enzyme labels. In this chapter, we will study the protein adsorption onto the sensor surface covered with self-assembled polyelectrolyte thin film. Furthermore, immunosensing based on the selective affinity between IgG and anti-IgG is also discussed.

\subsection{Immobilization of proteins}

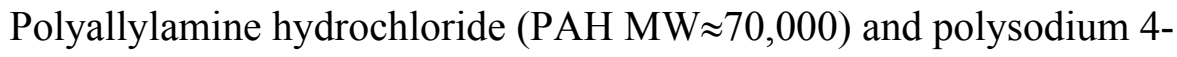

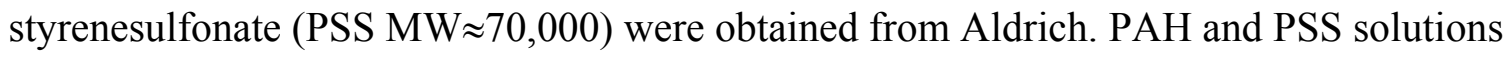
of $4 \mathrm{mg} / \mathrm{ml}$ each were prepared by dissolving polymer in ultrapure water (Nanopure, Barnstead). Both had salt $(\mathrm{NaCl})$ concentrations of $0.15 \mathrm{M} . \mathrm{pH}$ values were adjusted to 
7.5 (Corning Ion Analyzer 455). Pig IgG (1 mg/ml), anti-pig IgG $(0.5 \mathrm{mg} / \mathrm{ml})$ and antirabbit IgG $(0.5 \mathrm{mg} / \mathrm{ml})$ were supplied by Bethyl Inc. All antibodies and antigens were provided in buffered solutions. All experiments were carried out at room temperature.

The experimental setup of the multicavity FP sensor system is shown in Figure 5.1. The reflection spectrum of a multicavity FP sensor was monitored by a component testing system (CTS) (Micron Optics SI720) and recorded by a computer. A low noise fiber ring laser is included in the CTS as the light source. The analyzer in the CTS offers a measurement range of 1520-1570 nm with 1pm accuracy. A fiber coupler (AC Photonics) was connected to couple the light to the sensor and route the reflection signal back to the detector. This setup was used in the following sections.

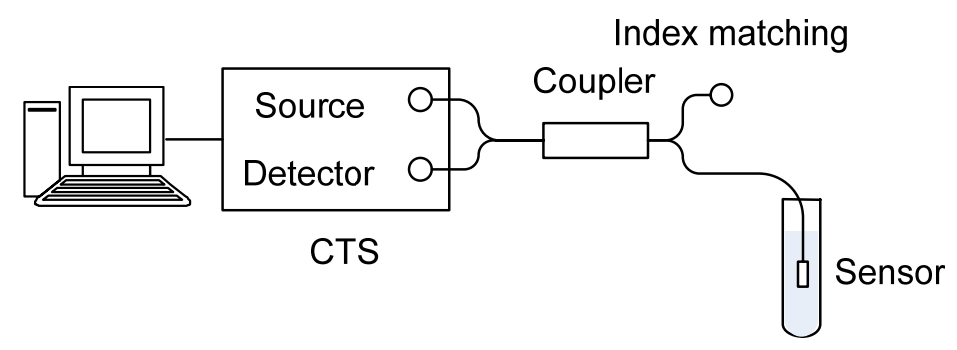

Figure 5.1 Schematic of the multicavity FP sensor system.

Polyelectrolyte films made up of cationic PAH and anionic PSS were alternately self-assembled onto a tubing-based multicavity sensor. In order to study the interactions between proteins and different surfaces, both a positively charged $[\mathrm{PAH} / \mathrm{PSS}]_{2} \mathrm{PAH}$ and a negatively charged $[\mathrm{PAH} / \mathrm{PSS}]_{2}$ film were built up. The reflection spectra corresponding to the multilayers were analyzed before protein adsorption. The thickness of the $[\mathrm{PAH} / \mathrm{PSS}]_{2} \mathrm{PAH}$ and $[\mathrm{PAH} / \mathrm{PSS}]_{2}$ films were $12.4 \pm 0.2 \mathrm{~nm}$ and $10.1 \pm 0.1 \mathrm{~nm}$ respectively $(\mathrm{n}=1.54)$. Sensors were then immersed in pig IgG solution $(20 \mu \mathrm{g} / \mathrm{ml}$ in $0.05 \mathrm{M}$ HEPES) for $60 \mathrm{~min}$. After immobilization of IgG, the reflection spectra of sensors were taken. The effective thickness of the $\mathrm{IgG}$ layer on the $[\mathrm{PAH} / \mathrm{PSS}]_{2} \mathrm{PAH}$ is $3.1 \pm 0.2 \mathrm{~nm}$ and on $[\mathrm{PAH} / \mathrm{PSS}]_{2}$ is $5.1 \pm 0.2 \mathrm{~nm}$, respectively ( $\mathrm{n}=1.432$ ). It is evident that the $\mathrm{IgG}$ adsorb on both positively and negatively charged multilayer surfaces because the surface domains 
of proteins have both positive and negative excess charges. The interpenetration of PAH and PSS layers allows some interactions with proteins no matter which one is the outermost layer. Moreover, polyclonal antibodies and antigens were used in our experiments. Polyclonal IgG or anti-IgG consists of many different molecules with distinct properties such as isoelectric point, hydrophobicity and structural stability. Although there is typically no definition of isoelectric point for polyclonal antibodies and antigens, the many clones in our samples would place the isoelectric point somewhere between 6.5 and 7.5 . The $\mathrm{pH}$ of the HEPES buffer $(0.05 \mathrm{M})$ is about 7.36 so the antibodies and antigens are weakly charged [205]. Hydrophobic interactions may become the major factor in the adsorption.

To determine the effect of IgG concentration on immobilization, a multicavity FP sensor with a self-assembled polymer film ([PAH/PSS $\left.]_{2}\right)$ was exposed to the HEPES buffer $(0.05 \mathrm{M})$. Polyclonal pig IgG solutions with different concentrations were prepared by injecting the IgG into the buffer. The change in thickness after a 60 min exposure as a function of IgG concentration is shown in Figure 5.2. From Figure 5.2, it is obvious that at lower concentrations $(<20 \mu \mathrm{g} / \mathrm{ml})$ the surface coverage is dependent on the concentration. At higher concentrations $(>20 \mu \mathrm{g} / \mathrm{ml})$ the surface coverage is saturated and essentially independent of the concentration. The slight increase for the higher concentration may come from the formation of multilayers. The experimental data were fitted to the Langmuir model [206]:

$$
\Gamma_{r}=\frac{\Delta d}{\Delta d_{m}}=\frac{k c}{1+k c},
$$

where $\Gamma_{r}$ is the relative surface coverage, $\Delta d$ is the thickness change from the adsorption, $\Delta d_{m}$ is the maximum change in thickness, $k$ is the binding constant, and $c$ is the bulk concentration of the protein in solution. After analyzing the data with nonlinear regression, it is often useful to display the data as a Scatchard plot (Figure 5.2), which is a linearization of Eq 5.1:

$$
\frac{\Delta d}{c}=k\left(\Delta d_{m}-\Delta d\right)
$$




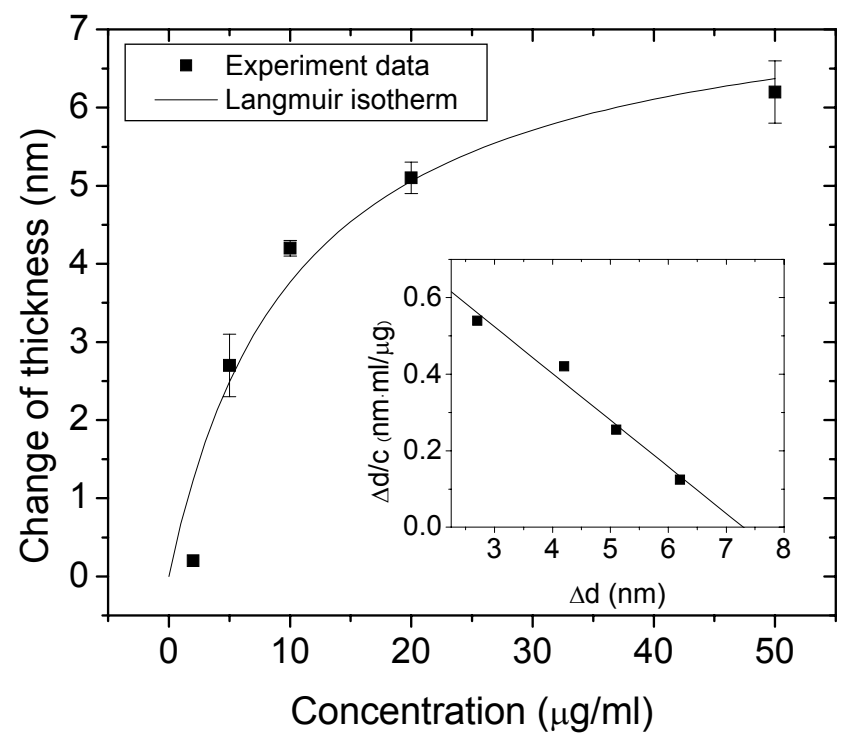

Figure 5.2 Change in thickness after 60 min exposure of the polymer surface to pig IgG as a function of protein concentration. Standard errors are calculated based on multiple tests. Inset is the Scatchard plot which is a linearization of the Langmuir model.

It is possible to estimate $\Delta d_{m}$ and $k$ from the Scatchard plot: $\Delta d_{m}$ is the x intercept and $k$ is the negative slope. Because of the distortion of the Scatchard transformation, linear regression of Scatchard transformed data may be far from their true value. Nonlinear regression of the data gives better estimation of saturation binding data: $k=0.10 \pm 0.04 \mathrm{ml} / \mu \mathrm{g}$ and $\Delta d_{m}=7.7 \pm 1.2 \mathrm{~nm}$.

The Langmuir model describes the binding of proteins to spatially fixed independent and identical binding sites. However the actual number of binding sites is modulated by the repulsion between the proteins. The extended volume of the adsorbed proteins must be included in the formalism. Under these circumstances, the accessible free surface is smaller than the assumption in Langmuir model. Although the theoretical basis of the Langmuir model is not strictly applicable to protein adsorption, it is convenient to estimate the maximum changes and assess the adsorption affinity from the 
initial slope. The thickness of IgG immobilized on $[\mathrm{PAH} / \mathrm{PSS}]_{2}$ is $5.1 \pm 0.2 \mathrm{~nm}(20 \mu \mathrm{g} / \mathrm{ml})$. The dimensions of $\mathrm{IgG}$ are approximately $10 \mathrm{~nm}$ in width, $14 \mathrm{~nm}$ in length and $5 \mathrm{~nm}$ in thickness [207]. The actual thickness of the immobilized layer will depend not only on the dimensions but also on the orientation and deformation of the IgG. In Figure 5.2, it is evident that at higher concentrations the thickness changes are about the thickness of an IgG monolayer.

In protein adsorption studies, the mass of protein adsorbed per adsorbent surface area (surface concentration $\Gamma$ ) is widely used. To estimate the adsorbed mass of $\operatorname{IgG}$ in our study, the surface concentration $\Gamma$ can be expressed as [208]:

$$
\Gamma=\frac{d\left(n-n_{b}\right)}{d n / d c},
$$

where $d$ is the thickness of the adsorbed protein, $n$ is the refractive index of the protein layer, $n_{b}$ is the refractive index of the protein solution. Here $n=1.432, n_{b}=1.335$ [175]. The refractive index increment $d n / d c$ of the IgG solution is $0.187 \mathrm{~cm}^{3} / \mathrm{g}$ [209]. Thus the surface concentration is $2.6 \pm 0.1 \mathrm{mg} / \mathrm{m}^{2}(20 \mu \mathrm{g} / \mathrm{ml})$.

\subsection{Antigen binding to immobilized monolayer $\operatorname{IgG}$}

The immunoreaction between $\operatorname{IgG}$ and anti-IgG was examined by multicavity FP sensors. Multicavity FP sensors coated with self-assembled polymer films ([PAH/PSS $]_{2}$ ) were first exposed to pig IgG solution ( $20 \mu \mathrm{g} / \mathrm{ml}$ in $0.05 \mathrm{M}$ HEPES). After immobilization of IgG, sensors were immersed in anti-pig IgG solutions with different concentrations ( 0 to $20 \mu \mathrm{g} / \mathrm{ml})$ for $60 \mathrm{~min}$. Anti-IgG solutions were prepared in HEPES buffer $(0.05 \mathrm{M})$.

The change in thickness from the binding of anti-IgG to the adsorbed $\mathrm{IgG}$ as a function of anti-IgG concentration is shown in Figure 5.3. Similar to the analysis of $\operatorname{IgG}$ binding on polymer, the experimental data were fitted by Langmuir model where $k=2.7 \pm 0.3 \mathrm{ml} / \mu \mathrm{g}$ and $\Delta d_{m}=5.37 \pm 0.08 \mathrm{~nm}$. It is obvious that the affinity constant of IgG to anti-IgG is much higher than that of IgG with polymer due to the immunoreactions. The thickness change is saturated at concentrations higher than $5 \mu \mathrm{g} / \mathrm{ml}$, compared with about $20 \mu \mathrm{g} / \mathrm{ml}$ for IgG adsorbed on polymer film. The dynamic range is limited by the high 
binding affinity. A kinetic experiment will solve the problem by monitoring the reaction constants. The thickness of anti-IgG bound to $\operatorname{IgG}$ is $5.32 \pm 0.05 \mathrm{~nm}(20 \mu \mathrm{g} / \mathrm{ml})$. The adsorption of $\operatorname{IgG}$ and binding of anti-IgG produce similar thickness changes due to the similar size of the molecules [210]. The surface concentration can be calculated from Eq 5.3 as $2.76 \pm 0.03 \mathrm{mg} / \mathrm{m}^{2}$. The binding ratio, or ratio of surface concentration of adsorbed anti-IgG to $\operatorname{IgG}$, was calculated as $1.06 \pm 0.05$. The binding ratio higher than one indicates some level of nonspecific binding since the anti-IgG may bind to other sites in addition to the IgG.

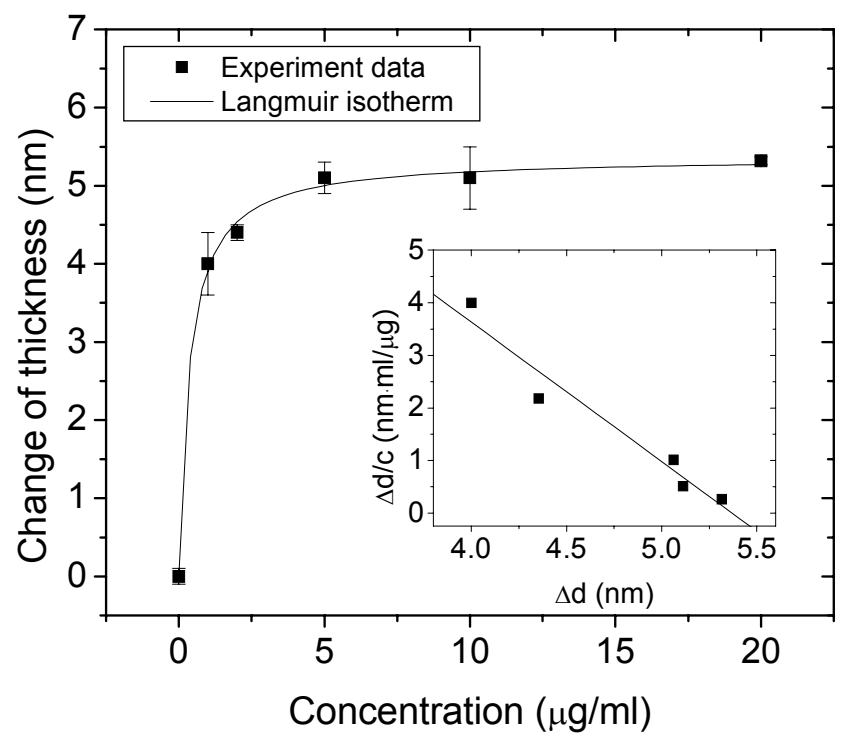

Figure 5.3 Change in thickness after $60 \mathrm{~min}$ of exposure of the $\operatorname{IgG}$ to anti-IgG as a function of protein concentration ( $\mathrm{n}=1.432)$. Inset is the Scatchard plot which is a linearization of the Langmuir model.

\subsection{Antigen binding to immobilized multilayer IgG}

It has been reported that embedded proteins kept their biological activity with respect to the immunological reaction even when thin polyelectrolyte layers were deposited over them [175]. The activity was found to be additive when a multilayer of 
antibodies were inserted between polyelectrolyte layers. This effect provides the potential to design bioactive materials with different biological responses.

Multilayer polyelectrolyte/IgG films were prepared by first assembling a $[\mathrm{PAH} / \mathrm{PSS}]_{2}$ film on the sensor surface. The surfaces were then alternately immersed in $\operatorname{IgG}$ solution for $60 \mathrm{~min}$ and PSS for 5min with rinsing and drying between each deposition. The PSS solution contained $0.15 \mathrm{M} \mathrm{NaCl}$. Figure 5.4 shows the change in optical thickness during the fabrication of an $\mathrm{IgG} / \mathrm{PSS}$ multilayer on a $[\mathrm{PAH} / \mathrm{PSS}]_{2}$ precursor film. The changes in thickness due to IgG layers gradually increased as listed in Table 5.1. This may come from the penetration of $\operatorname{IgG}$ into the PSS layer or the aggregation of IgG. The thickness decrease from some PSS layers may be attributable to desorption of IgG in the PSS solution because of the higher ionic strength [197]. The penetration or aggregation of $\mathrm{IgG}$ provides a greater surface area for immobilization. The immunologic response of the multilayer assembly was assessed by interaction with the corresponding anti-IgG. The change in thickness from the binding of anti-IgG $(20 \mu \mathrm{g} / \mathrm{ml})$ onto the multilayers $[\mathrm{IgG} / \mathrm{PSS}]_{4}$ is $8.9 \mathrm{~nm}$, significantly higher than the thickness change from an IgG monolayer $(5.3 \mathrm{~nm})$. This result demonstrates the feasibility of increasing the binding capacity of organic films over a conventional monolayer film on immunosensors. 


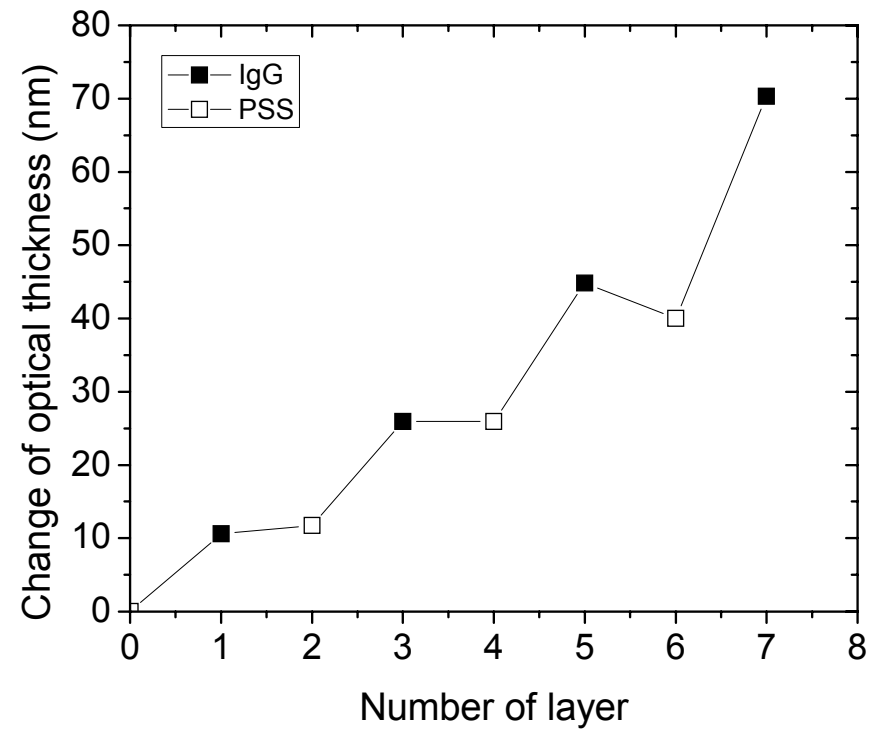

Figure 5.4 Change in optical thickness with the IgG/PSS multilayer formed on $[\mathrm{PAH} / \mathrm{PSS}]_{2}$ film.

Table 5.1 Change in thickness of IgG layer $(\mathrm{n}=1.432)$.

\begin{tabular}{|c|c|c|c|c|}
\hline Layer number & 1 & 2 & 3 & 4 \\
\hline Thickness $(\mathrm{nm})$ & 7.4 & 9.9 & 13.2 & 21.2 \\
\hline
\end{tabular}

\subsection{Nonspecific binding of anti-IgG}

In addition to binding of receptors of interest, analytes may also bind to other sites, which is called nonspecific binding. Specificity is a key issue in immunosensing. A sensor coated with IgG may bind other kinds of anti-IgG nonspecifically. If a high background is present from nonspecific binding, pre-treatment of the sensor with binding block solution may be required.

Nonspecific binding between $\operatorname{IgG}$ and noncorrelated anti-IgG was examined by a multicavity sensor. The sensor was first coated with a polymer precursor film ([PAH/PSS $\left.]_{5} \mathrm{PAH}\right)$. Then it was immersed in sheep IgG solution $(50 \mu \mathrm{g} / \mathrm{ml}$ in $0.05 \mathrm{M}$ 
HEPES) for $1 \mathrm{~h}$. After rinsing and drying, the sensor was immersed in anti-rabbit IgG solution ( $25 \mu \mathrm{g} / \mathrm{ml}$ in $0.05 \mathrm{M}$ HEPES) for $1 \mathrm{~h}$. Figure 5.5 shows the changes in the optical thickness of the fiber-film cavity. Immobilization of the sheep IgG resulted in a change of $11.6 \mathrm{~nm}$ with a refractive index assumption of 1.432 [175]. The nonspecific binding of anti-rabbit IgG had a change of $5.6 \mathrm{~nm}(\mathrm{n}=1.432)$ which is obvious in Figure 5.5. This nonspecific binding may due to the adsorption of anti- $\operatorname{IgG}$ to the uncovered precursor film. Therefore it is necessary to reduce the nonspecific binding by a blocking process. A binding blocking process uses a concentrated protein solution (e.g. ovalbumin, BSA, haemoglobin, non-fat milk powder) to prevent further non-specific binding of proteins.

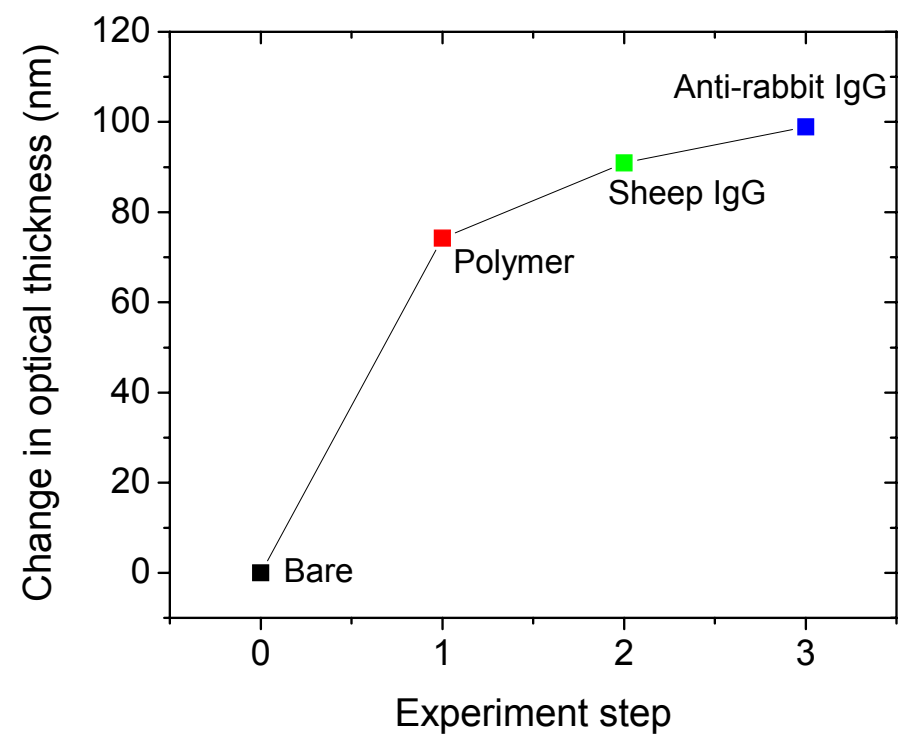

Figure 5.5 Optical thickness changes in the fiber-film cavity during self-assembly of the polymer film, immobilization of sheep IgG and nonspecific binding with anti-rabbit IgG.

Another test was carried out to investigate the blocking effect. After self-assembly of polymer film with structure $[\mathrm{PAH} / \mathrm{PSS}]_{2}$, a multicavity sensor was immersed in pig IgG solution $(50 \mu \mathrm{g} / \mathrm{ml}$ HEPES $0.05 \mathrm{M})$ for $1 \mathrm{~h}$. The reflection spectrum was recorded after cleaning and drying. The increase in optical thickness shown in Figure 5.6 reflects 
immobilization of $\operatorname{IgG}$ on the sensor end. The thickness change is $5.9 \mathrm{~nm}(\mathrm{n}=1.432)$. In order to reduce nonspecific binding, the sensor was then immersed in a standard binding block solution [2\% bovine serum albumin (BSA)] for $1 \mathrm{~h}$, again increasing the optical thickness from the adsorption of BSA. A cross-reactivity experiment was then performed involving the immobilized pig IgG and anti-rabbit IgG or anti-pig IgG. The sensor with immobilized pig IgG was first immersed in anti-rabbit IgG $(25 \mu \mathrm{g} / \mathrm{ml}$ HEPES 0.05M) for $1 \mathrm{~h}$. After cleaning and drying, the reflection spectrum was acquired. The sensor was then immersed in anti-pig IgG $(25 \mu \mathrm{g} / \mathrm{ml}$ HEPES $0.05 \mathrm{M})$ for $1 \mathrm{~h}$. Another spectrum was taken after cleaning and drying. In Figure 5.6, the cross interaction between the anti-rabbit IgG and the immobilized pig IgG yields a total thickness change of less than $0.1 \mathrm{~nm}$ $(\mathrm{n}=1.432)$. The binding of anti-pig IgG to immobilized pig IgG yields a thickness change of $5.7 \mathrm{~nm}(\mathrm{n}=1.432)$. The difference in binding anti-rabbit IgG and anti-pig IgG to immobilized pig IgG reflects that the nonspecific binding level is much lower than that of the specific binding.

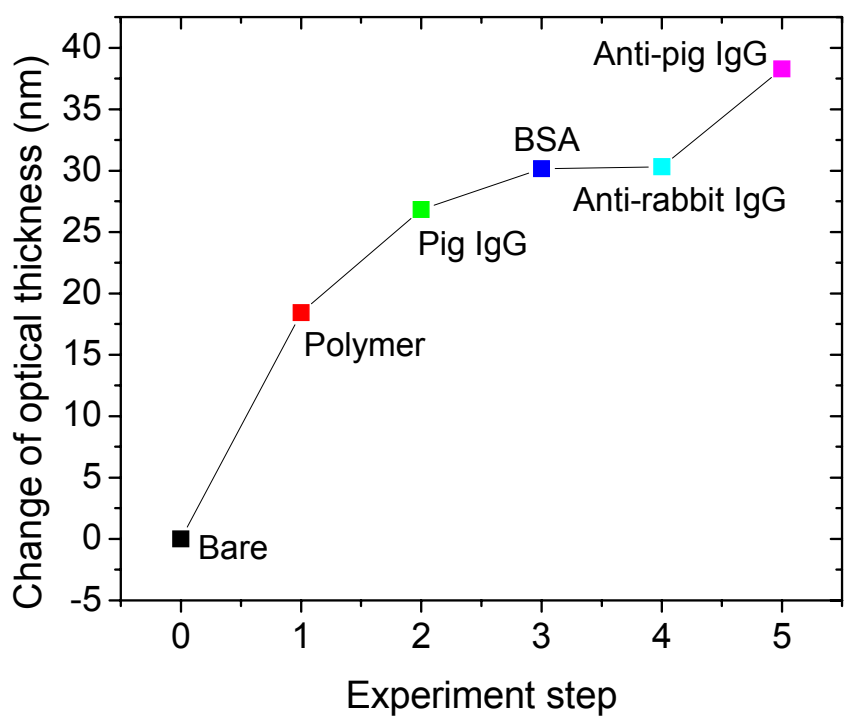

Figure 5.6 Optical thickness changes in the fiber-film cavity during self-assembly of the polymer film, immobilization of pig IgG, binding block, nonspecific binding with antirabbit $\operatorname{IgG}$ and immunosensing of anti-pig IgG. 
The nonspecific binding between rabbit $\operatorname{IgG}$ and anti-pig $\operatorname{IgG}$ was also examined by a similar experiment. The sensor coated with polymer film $[\mathrm{PAH} / \mathrm{PSS}]_{2}$ was first immersed in rabbit IgG solution ( $50 \mu \mathrm{g} / \mathrm{ml}$ HEPES $0.05 \mathrm{M})$ for $1 \mathrm{~h}$. There is a thickness change of $6.8 \mathrm{~nm}$ for immobilization of rabbit $\operatorname{IgG}(\mathrm{n}=1.432)$. Then the sensor was treated with binding block ( $2 \% \mathrm{BSA})$ for $1 \mathrm{~h}$, causing an increase in the optical thickness from the adsorption of BSA. The nonspecific binding between the immobilized rabbit IgG and anti-pig IgG was investigated by dipping the sensor in anti-pig $\operatorname{IgG}(25 \mu \mathrm{g} / \mathrm{ml}$ HEPES $0.05 \mathrm{M})$ for $1 \mathrm{~h}$. The sensor was then immersed in anti-rabbit $\operatorname{IgG}(25 \mu \mathrm{g} / \mathrm{ml}$ HEPES $0.05 \mathrm{M}$ ) for another hour. In Figure 5.7, the cross interaction between the anti-pig IgG and the immobilized rabbit IgG yields a thickness change of around $1.2 \mathrm{~nm}$ $(\mathrm{n}=1.432)$. However, the specific binding between anti-rabbit IgG and rabbit IgG yields a thickness change of $7.5 \mathrm{~nm}(\mathrm{n}=1.432)$. Again the difference in binding anti-pig IgG and anti-rabbit IgG to immobilized rabbit IgG reflects that the nonspecific binding level is much lower than that of the specific binding. These results strongly suggest that bindingblock process will efficiently suppress the nonspecific binding. 


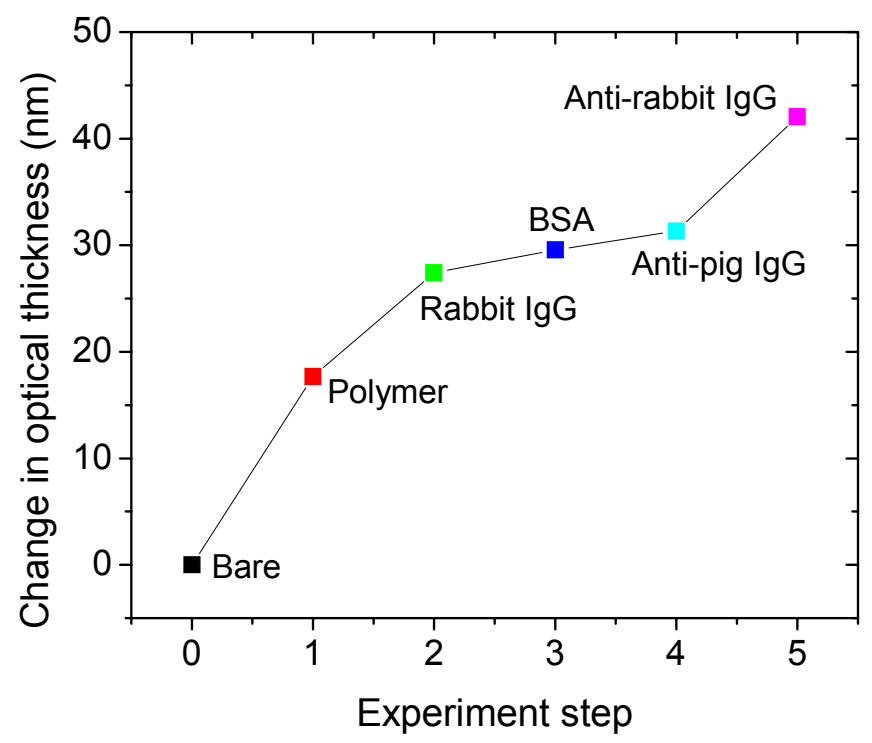

Figure 5.7 Optical thickness changes in the fiber-film cavity during self-assembly of the polymer film, immobilization of rabbit IgG, binding block, nonspecific binding with antipig IgG and immunosensing of anti-rabbit IgG.

\subsection{Binding kinetics}

\subsubsection{Introduction}

As mentioned in section 5.3, the dynamic range of the immunosensor was limited in steady-state measurements due to the high affinity between antibody and antigen. If the sensor can be applied in a solution environment, kinetic measurements will reveal more details of immunoreactions such as rate constant. Analysis of the real-time binding profile will also provide a fast and effective way of measuring antigen concentration [207].

In order to characterize the binding between antibody and antigen, both the equilibrium binding constant $K$ and two reaction rate constants $k_{a}$ and $k_{d}$ are of particular interest. The equilibrium constant $K$ describes the ratio between bound and unbound antibody for a certain concentration of antigen under steady-state conditions. It represents 
the binding behavior at equilibrium. $k_{a}$ and $k_{d}$ are the association rate and dissociation rate of the antibody-antigen complex. The binding constant and kinetic rate constants can be described by a simplified Langmuir model in which the binding sites on the surface are independent and the transport of antigens to the surface is not affecting the reaction rates. Here we assume that antibodies are immobilized on the surface and antigens are available in the solution for binding. The reaction between antibodies of concentration $[A b]$ and antigens of concentration $[\mathrm{Ag}]$ can be expressed as [202]:

$$
[A b]+[A g] \underset{k_{d}}{\stackrel{k_{a}}{\rightleftharpoons}}[A b \cdot A g],
$$

where $k_{a}$ and $k_{d}$ are the kinetic rate constants of association and dissociation. [Ab·Ag] is the concentration of antibody-antigen complex. The binding constant $K$ at equilibrium is defined as:

$$
K=\frac{[A b \cdot A g]}{[A b][A g]} .
$$

The total number of immobilized antibodies on the surface is

$$
\left[A b_{t}\right]=[A b]+[A b \cdot A g] .
$$

The relative surface coverage according to the Langmuir model is

$$
\Gamma_{r}=\frac{[A b \cdot A g]}{\left[A b_{t}\right]}=\frac{K[A g]}{1+K[A g]} .
$$

The reaction rate can be described by:

$$
\frac{d[A b \cdot A g]}{d t}=k_{a}[A b][A g]-k_{d}[A b \cdot A g] .
$$

At equilibrium $d[A b \cdot A g] / d t=0$, so the binding constant is the ratio of the rate constants $k_{a}$ and $k_{b}$

$$
K=\frac{k_{a}}{k_{d}} .
$$

Substitute Eq 5.6 in Eq 5.8, then Eq 5.8 can be expressed as:

$$
\frac{d[A b \cdot A g]}{d t}=k_{a}\left[A b_{t}\right][A g]-\left(k_{a}[A g]+k_{d}\right)[A b \cdot A g] .
$$


It is assumed that the antigen concentration $[\mathrm{Ag}]$ is not affected by the binding reaction. The concentration of complexes can be derived as

$$
[A b \cdot A g]=\frac{k_{a}\left[A b_{t}\right][A g]}{k_{a}[A g]+k_{d}}\left(1-\exp \left(-\left(k_{a}[A g]+k_{d}\right) t\right)\right) \text {. }
$$

Obviously the relative surface coverage in Eq 5.7 represents the response for infinite time

$$
[A b \cdot A g]_{t \rightarrow \infty}=\frac{K\left[A b_{t}\right][A g]}{1+K[A g]} .
$$

In our study, the bound complex $[\mathrm{Ab} \cdot \mathrm{Ag}]$ was measured as a function of the antigen concentration in solution at steady state. $\left[A b_{t}\right]$ and $\mathrm{K}$ are obtained from fitting the relative surface coverage with the antigen concentration. Considerable time is needed in a steady-state experiment to determine the binding constant. A kinetic experiment will take less time and be able to determine the rate constants and binding constant simultaneously. The binding reaction will be monitored in situ for different antigen concentrations.

\subsubsection{Theoretical model for kinetic measurement}

The thin-film sensing is based on the measurement of optical thickness changes of the fiber-film cavity caused by the adsorption of target molecules. The adsorbed thin film can be estimated as an extension of the fiber cavity when the refractive index of the film is similar to the fiber. This assumption is valid since the polymer film and biomolecular layer have similar refractive indices with the optical fiber. When the measurement is ex situ, i.e. in the air, the reflection from the fiber-film interface is much smaller than the reflection from fiber-air or film-air interfaces. For example, the reflection coefficient between a fiber $(n=1.45)$ and a polymer film $(n=1.54)$ is only $2 \%$ of that from the film-air interface, and $3 \%$ of that from the fiber-air interface. In the simplified analysis, the adsorbed film can be treated as part of the fiber-film cavity. The changes in the optical thickness of the fiber-film cavity reflect the adsorption process. As described in Chapter 4, similar results were achieved by adsorption of polymer self-assembly on fibers and FP sensors, which confirmed the above assumption. However, the weak reflection at the 
fiber-film interface can not be neglected if outer medium has a refractive index similar to the film. Therefore, a different theoretical analysis is required for in situ applications.

A theoretical model for the tubing-based multicavity sensor is shown in Figure 5.8. Following the analysis by matrix optics in section 2.3 .2 , the composite cavity matrix can be expressed as

$$
S=H_{12} L_{2} H_{23} L_{3} H_{34} L_{4} H_{45} .
$$

The interface transition matrices are

$$
\begin{aligned}
& H_{12}=\frac{1}{\tau_{12}}\left(\begin{array}{cc}
1 & \rho_{12} \\
\rho_{12} & 1
\end{array}\right), H_{23}=\frac{1}{\tau_{23}}\left(\begin{array}{cc}
1 & \rho_{23} \\
\rho_{23} & 1
\end{array}\right), \\
& H_{34}=\frac{1}{\tau_{34}}\left(\begin{array}{cc}
1 & \rho_{34} \\
\rho_{34} & 1
\end{array}\right), H_{45}=\frac{1}{\tau_{45}}\left(\begin{array}{cc}
1 & \rho_{45} \\
\rho_{45} & 1
\end{array}\right) .
\end{aligned}
$$

The layer propagation matrices are

$$
L_{j}=\left(\begin{array}{cc}
\exp \left(-i \beta_{j}\right) & 0 \\
0 & \exp \left(i \beta_{j}\right)
\end{array}\right), j=2,3,4
$$

where $\beta_{2}=\frac{2 \pi n_{2} d_{2}}{\lambda}+\phi_{0}{ }^{\prime}, \beta_{3}=\frac{2 \pi n_{1} d_{3}}{\lambda}, \beta_{4}=\frac{2 \pi n_{3} d_{4}}{\lambda}$ are the phase shifts of the air cavity, fiber cavity and film. $n_{1}=1.45, n_{2}=1$ are the refractive indices of fiber and air. The refractive index of the film $\left(n_{3}\right)$ depends on the film species. $\phi_{0}$ is the coupling phase shift from the longitudinal misalignment. The reflectance of the multicavity sensor is

$$
R=|\rho|^{2}=\left|\frac{S_{12}}{S_{22}}\right|^{2},
$$

where $\rho$ is the reflection coefficient. $S_{12}$ and $S_{22}$ are the components of the stack matrix and can be described as

$$
\begin{aligned}
& \mathrm{S}_{12}=\left\{\rho_{12} \exp \left[i\left(\beta_{2}+\beta_{3}+\beta_{4}\right)\right]+\rho_{23} \exp \left[-i\left(\beta_{2}-\beta_{3}-\beta_{4}\right)\right]+\right. \\
& \rho_{34} \exp \left[-i\left(\beta_{2}+\beta_{3}-\beta_{4}\right)\right]+\rho_{45} \exp \left[-i\left(\beta_{2}+\beta_{3}+\beta_{4}\right)\right]+ \\
& \rho_{12} \rho_{23} \rho_{34} \exp \left[i\left(\beta_{2}-\beta_{3}+\beta_{4}\right)\right]+\rho_{12} \rho_{23} \rho_{45} \exp \left[i\left(\beta_{2}-\beta_{3}-\beta_{4}\right)\right]+ \\
&\left.\rho_{12} \rho_{34} \rho_{45} \exp \left[i\left(\beta_{2}+\beta_{3}-\beta_{4}\right)\right]+\rho_{23} \rho_{34} \rho_{45} \exp \left[-i\left(\beta_{2}-\beta_{3}+\beta_{4}\right)\right]\right\} / \tau_{12} \tau_{23} \tau_{34} \tau_{45},
\end{aligned}
$$




$$
\begin{aligned}
\mathrm{S}_{22}=\left\{\exp \left[i\left(\beta_{2}+\beta_{3}+\beta_{4}\right)\right]+\rho_{12} \rho_{23} \exp \left[-i\left(\beta_{2}-\beta_{3}-\beta_{4}\right)\right]+\right. & \\
& \rho_{12} \rho_{34} \exp \left[-i\left(\beta_{2}+\beta_{3}-\beta_{4}\right)\right]+\rho_{12} \rho_{45} \exp \left[-i\left(\beta_{2}+\beta_{3}+\beta_{4}\right)\right]+ \\
& \rho_{23} \rho_{34} \exp \left[i\left(\beta_{2}-\beta_{3}+\beta_{4}\right)\right]+\rho_{23} \rho_{45} \exp \left[i\left(\beta_{2}-\beta_{3}-\beta_{4}\right)\right]+ \\
& \left.\rho_{34} \rho_{45} \exp \left[i\left(\beta_{2}+\beta_{3}-\beta_{4}\right)\right]+\rho_{12} \rho_{23} \rho_{34} \rho_{45} \exp \left[-i\left(\beta_{2}-\beta_{3}+\beta_{4}\right)\right]\right\} / \tau_{12} \tau_{23} \tau_{34} \tau_{45},
\end{aligned}
$$

where $\rho_{i j}$ and $\tau_{i j}$ are the reflection coefficient and transmission coefficient of interface i$\mathrm{j}$ respectively. When the reflection at the fiber-film interface can be neglected, the reflectance of the multicavity sensor can be simplified as Eq. 2.50, which is the case where the measurement is carried out in the air after equilibrium. For kinetic measurement, the rigorous form in Eq. 5.16, 5.17 and 5.18 was applied.

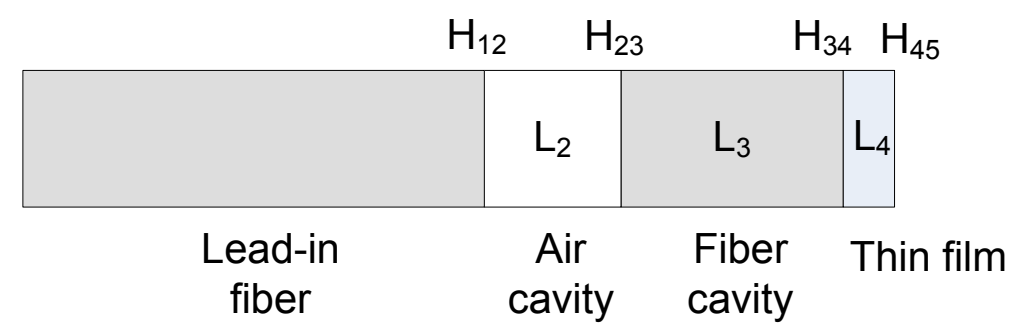

Figure 5.8 Theoretical model for tubing-based multicavity sensor.

\subsubsection{Experiment results and analysis}

\subsubsection{Antigen binding to immobilized $\operatorname{Ig} G$}

After immobilization of IgG onto the polymer modified sensor, the kinetics of the anti-IgG binding to the immobilized IgG layer were determined. Changes in optical thickness for the binding of anti-pig IgG to pig IgG are shown in Figure 5.9. The change for the low concentration (i.e. $1 \mu \mathrm{g} / \mathrm{ml}$ ) is almost linear with time and equilibrium was not achieved in $60 \mathrm{~min}$. The change is more rapid for higher anti-IgG concentrations. Equilibrium is observed for concentrations above $20 \mu \mathrm{g} / \mathrm{ml}$ in $60 \mathrm{~min}$. 


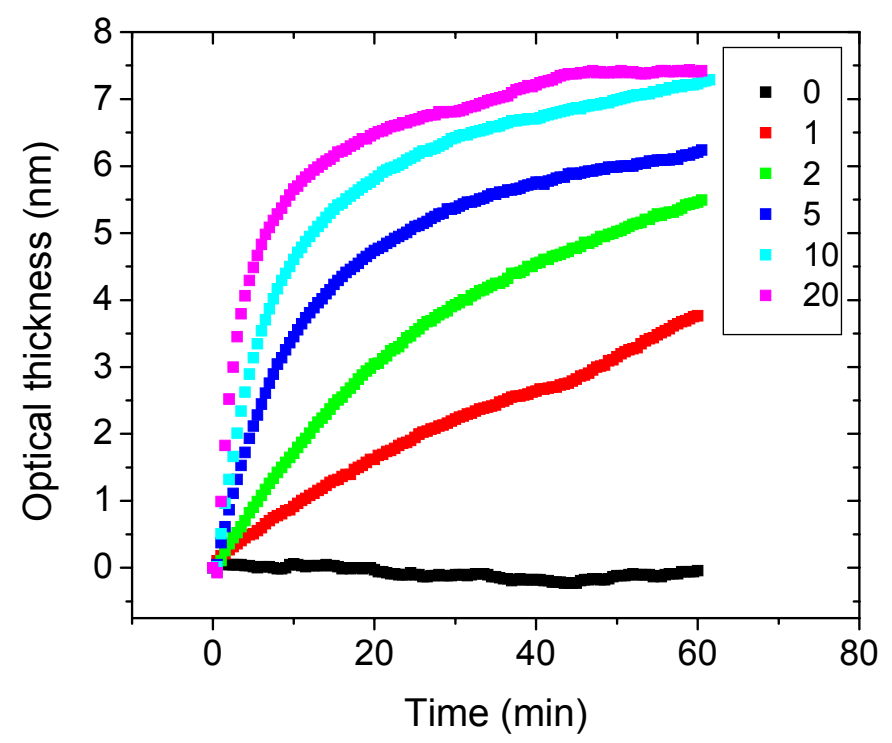

Figure 5.9 Changes in optical thickness during binding of anti-pig IgG at various concentrations $(\mu \mathrm{g} / \mathrm{ml})$ to immobilized pig IgG.

The kinetics of immobilization of biomolecules can be analyzed by Langmuir model [211]. As described in Eq. 5.11, a fitting algorithm from the simple adsorption model was used,

$$
L=L_{m}[1-\exp (-b t)],
$$

where $L$ is the measured optical thickness, $L_{m}$ is the optical thickness at steady state, $b$ is the rate constant, and $t$ is the time elapsed after the sensor contacting with anti-IgG. Figure 5.10 shows the theoretical fitting of the kinetic measurement. The deviation between the model and experiment data is obvious because the Langmuir model only applies to biomolecular adsorption where each binding site is equivalent and independent from neighboring sites. The model may be applicable for low surface coverage, but is unlikely to describe the adsorptions over a wide range of surface coverage. Various adsorption models have been developed for better understanding of the adsorption kinetics [212-214]. Rahn and Hallock presented an empirical exponential model for the binding kinetics [213]: 


$$
\Gamma(t)=\Gamma_{f}\left[1-\exp \left(-k t^{\alpha}\right)\right]
$$

where $\Gamma$ is the surface concentration, $\Gamma_{f}$ is the maximum coverage for different configurations of adsorbed species at various concentrations, $k$ and $\alpha$ are the fitting parameters which vary with solution concentrations. A similar model for changes in optical thickness can be derived as:

$$
L=L_{m}\left[1-\exp \left(-k t^{\alpha}\right)\right]
$$

Figure 5.11 shows an example of the fitting with the above exponential model. By comparing the coefficients of determination $\left(R^{2}\right)$, the empirical model $\left(R^{2}=0.98\right)$ has a better fit than the simple Langmuir model $\left(\mathrm{R}^{2}=0.95\right)$.

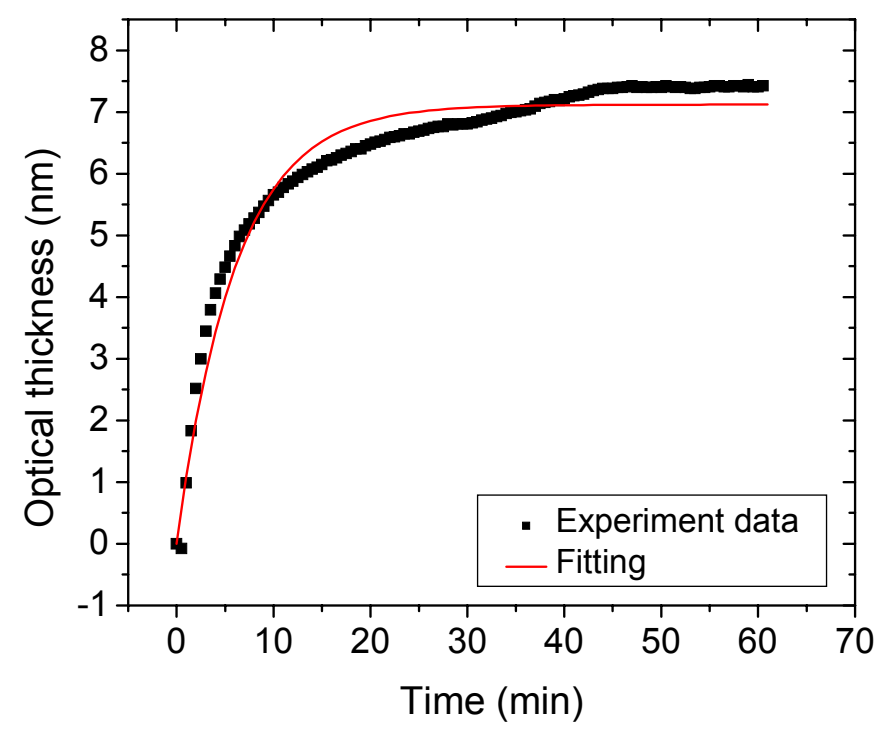

Figure 5.10 Theoretical fitting of binding anti-pig $\operatorname{IgG}(20 \mu \mathrm{g} / \mathrm{ml})$ to immobilized pig IgG by Langmuir model. 


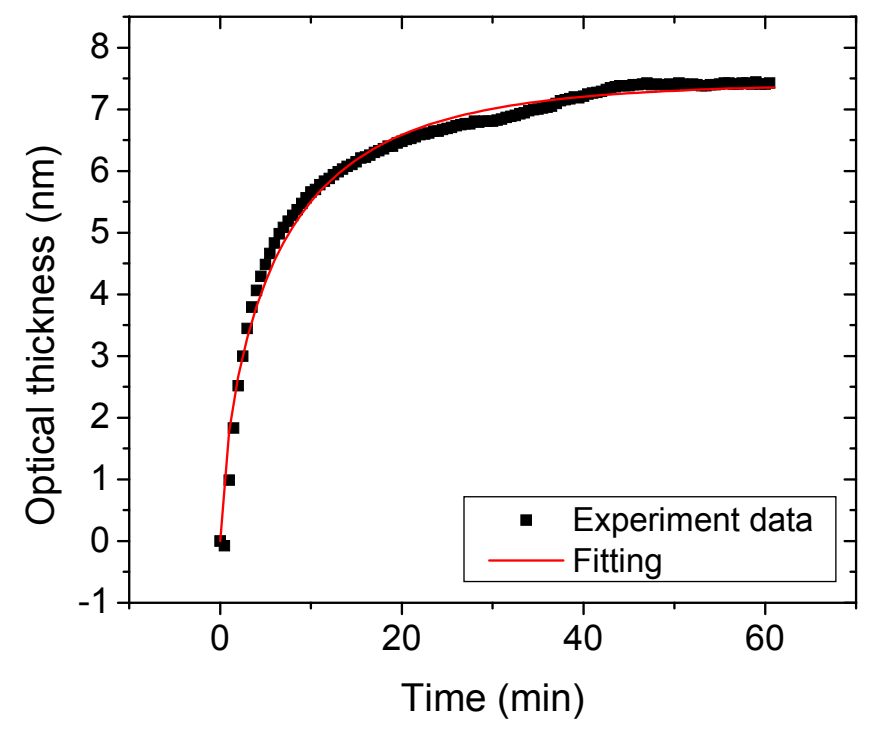

Figure 5.11 Theoretical fitting of binding anti-pig $\operatorname{IgG}(20 \mu \mathrm{g} / \mathrm{ml})$ to immobilized pig $\operatorname{IgG}$ by empirical model.

\subsubsection{Rate of change in optical thickness with antigen concentration}

The initial rate of change in optical thickness with anti-pig IgG concentration is shown in Figure 5.12. The rate is almost linearly related to the anti-IgG concentration, which is in agreement with other work [207]. From Eq. 5.10, the initial reaction rate can be derived as:

$$
\left.\frac{d[A b \cdot A g]}{d t}\right|_{t=0}=k_{a}\left[A b_{t}\right][A g],
$$

which is proportional to the antigen concentration. The above relationship is obtained from the Langmuir model, which only applies to biomolecular adsorption where each binding site is equivalent and independent from neighboring sites. The nonlinearity of higher concentrations may come from the diffusion-controlled kinetics, where the concentration of the adsorbing molecules adjacent to the surface will be much lower than the bulk concentration of this species [214]. The initial rate of change in the optical thickness provides a fast and effective way to measure the concentration of adsorbing 
species. It is not only useful for low concentrations, where a relative small spread in the rate data is found compared with that obtained from steady-state data, but also applicable for high concentrations, avoiding the saturation in the steady state. Thus binding kinetics provides larger dynamic range than the equilibrium methods.

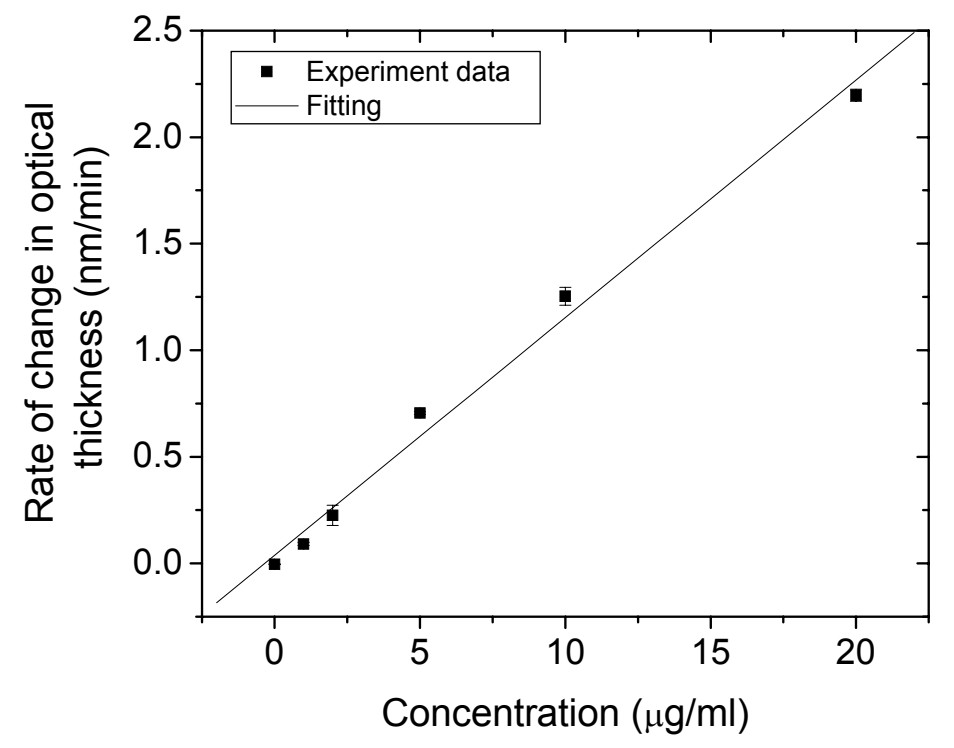

Figure 5.12 Rate of change in optical thickness as a function of anti-pig IgG concentration for binding to pig IgG.

\subsubsection{Temperature compensation}

In previous sections, tubing-based MFPI sensors have been applied in biosensing. Under temperature variations, thermal-induced errors can not be neglected due to the significant influence on the measurement accuracy. However, the temperature resolution of the tubing-based MFPI sensor is only about $2^{\circ} \mathrm{C}$ because of the low thermal expansion coefficient of the silica tubing (section 2.4). This limit the application of tubing-based sensors in temperature-compensated biosensing since the temperature variation is usually small, which is required by the biological reactions. The microgap multicavity sensor 
composed of a temperature sensor and a thin-film sensor increases the temperature sensitivity by an order of magnitude and has demonstrated its capability in thin-film sensing (Section 3.4). Therefore we applied the microgap MFPI sensor in biosensing under temperature variations.

The experiment setup of the microgap sensor system is similar to the description in section 3.4. As shown in Figure 5.13, the environmental temperature of the sensor was controlled by a water bath. A small vial $(0.5 \mathrm{ml})$ containing the solution was immersed in the water. The water temperature was controlled by a hot plate.

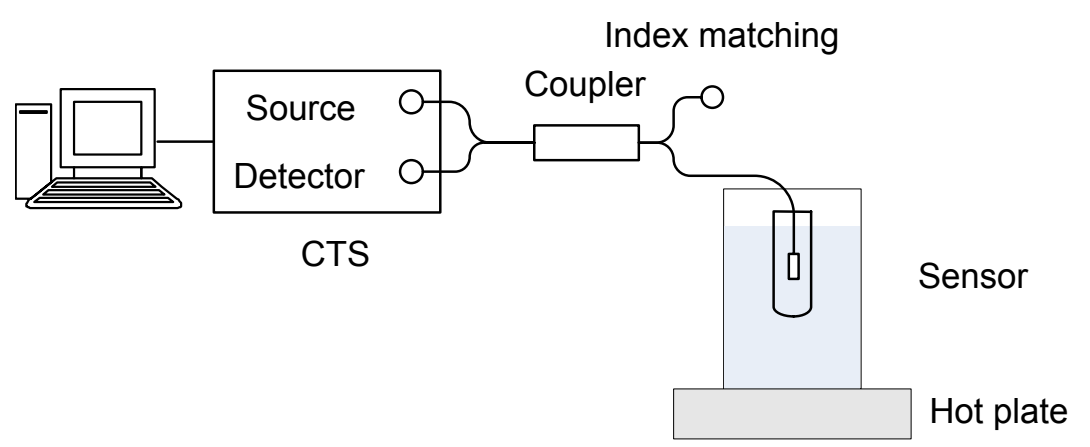

Figure 5.13 Schematic of microgap multicavity FP sensor system.

A microgap multicavity FP sensor coated with self-assembled polymer films ([PAH/PSS $]_{2}$ ) was first exposed to pig IgG solution ( $20 \mu \mathrm{g} / \mathrm{ml}$ in 0.05M HEPES). After immobilization of IgG, the sensor was immersed in anti-pig IgG solution $(20 \mu \mathrm{g} / \mathrm{ml}$ in $0.05 \mathrm{M}$ HEPES). The kinetics of the anti-IgG binding to the immobilized IgG layer were determined by changes in optical thickness as shown in Figure 5.14. Theoretical model in section 5.6.2 was applied for the analysis. The temperature of the anti-IgG solution was controlled by the water bath. The top curve shows the temperature changes during the test, which was measured by the built-in temperature sensor. In order to increase the temperature range to be investigated, the water temperature was first decreased to around $7^{\circ} \mathrm{C}$ in a refrigerator. Then during the immunosensing, the temperature was slowly increased to around $37^{\circ} \mathrm{C}$. The middle curve shows the change in optical thickness of the 
thin film without compensation. A total change of $36.9 \mathrm{~nm}$ was observed which is due to immunoreactions and thermal effects of the thin-film sensor. The optical thickness slowly increased and no equilibrium state was achieved within 60 min because of the thermal effects. The microgap sensor had been calibrated in the environmental chamber before coating. According to Eq 3.12, the changes in the optical cavity lengths of the temperature sensor and thin-film sensor have a linear relationship. Therefore the thermalinduced error in thin-film sensing can be easily compensated by comparing the changes in optical cavity lengths of two sensors. Temperature compensation revealed that the equilibrium state from the immunoreactions was actually attained within 20 min, as can be seen from the bottom curve in Figure 5.14. The change in optical thickness reduced to about $7.1 \mathrm{~nm}$, which corresponded to thickness changes of about $5.0 \mathrm{~nm}(\mathrm{n}=1.432)$. The thickness change is similar to the result under room temperature (Section 5.3), which demonstrates the capability of microgap MFPI sensor in biosensing with temperature variations. 

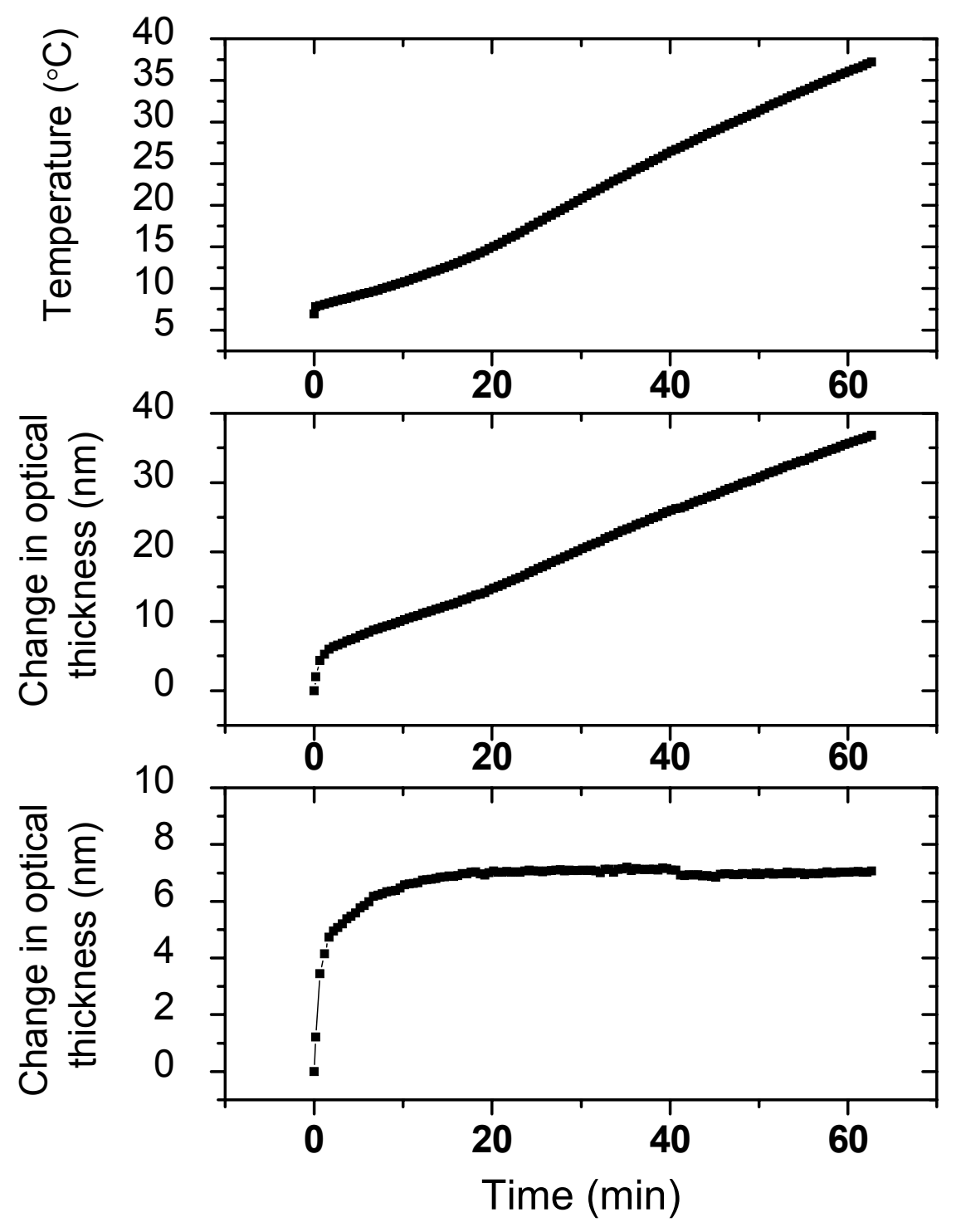

Figure 5.14 Effects of temperature compensation on the change in optical thickness during immunosensing. The top curve shows the temperature changes during the test. The middle curve shows the change in optical thickness before compensation and the lower curve shows the change after compensation. 


\subsection{Conclusions}

Electrostatic self-assembly provides an effective and convenient method for surface modification to retain the activity of proteins in fiber optic biosensors.

Comparison of the adsorption of IgG between positively and negatively charged polymer self-assemblies suggests that hydrophobic interactions may be the dominating factor for immobilization at the $\mathrm{pH}$ values investigated. The different thicknesses of the adsorbed layers on both types of films may be a reflection of the intensity of the hydrophobic interactions and the coexistence of positively charged and negatively charged proteins in the polyclonal antibodies. Due to the higher effective thickness of the adsorbed proteins, $[\mathrm{PAH} / \mathrm{PSS}]_{2}$ film was selected as the precursor film. The saturated immobilization of IgG onto polymer self-assembly was investigated. The thickness changes at higher concentrations $(>20 \mu \mathrm{g} / \mathrm{ml})$ are around the thickness of IgG monolayer.

The feasibility of using multicavity FP sensor as a biosensor has been verified. Proteins can keep their biological activity after immobilization onto the polymer selfassembly. The high affinity between IgG and corresponding anti-IgG assured the high sensitivity but limited the dynamic range in the steady-state measurement. The embedded proteins in multilayer films kept their biological activity even with polyelectrolyte layers over them. The additive activity for multilayer antibodies provides the potential to increase the sensitivity. Nonspecific binding was efficiently suppressed by introducing binding block before immunosensing.

A theoretical model of a multicavity sensor was developed for in situ application. Kinetic measurements reveal more details of immunoreactions such as rate constant. Analysis of the real-time binding profile will also provide a fast and effective way in the measurement of the antigen concentration. Furthermore, the binding kinetics provides a larger dynamic range than the steady-state methods.

The high temperature sensitivity of microgap MFPI sensors enables the biosensing under temperature variations. This greatly improves the flexibility in sample handling and provides the opportunity for investigation the temperature effect in biological reactions. 


\section{Chapter 6 Conclusions}

\subsection{Conclusions}

The fiber-optic sensing industry involves very diverse technologies and various applications. As one of the major technologies, fiber-optic Fabry-Perot interferometric (FFPI) sensors have been widely used due to their high sensitivity, ease of fabrication, localization and lead insensitivity. In this work, two types of fiber-optic multicavity Fabry-Perot interferometric (MFPI) sensors with built-in temperature compensation were designed and fabricated for thin-film measurement in chemical and biological areas.

A tubing-based MFPI sensor has been developed based on a hybrid FFPI sensor. The cavity lengths were controlled by cleaving and fusion-splicing techniques. The size of the sensor was made smaller than traditional extrinsic FFPI sensors by sandwiching a silica capillary tube between the lead-in fiber and the reflection fiber. An additional fiber cavity was introduced to interferometrically measure the adsorption of self-assembled thin-films. By matrix optics, a theoretical model of the multicavity sensor was developed which is in agreement with the experimental results. Each cavity length of the multicavity sensor can be measured with high resolution by demodulation of the reflection spectrum. Any variations to the end surface of the fiber cavity will change the reflection thus shifting the fringe pattern. Consequently, polymer self-assembly can be measured by the multicavity sensor. Further immobilization of proteins onto the sensor demonstrated its feasibility in biological applications. The temperature dependence of the fiber-cavity optical thickness can be compensated by extracting the temperature information from the air cavity, greatly reducing the temperature dependence of the thin-film measurement. This provides the opportunity to examine the thin-film characteristics under different environment temperatures.

To further increase the temperature sensitivity, a microgap FP sensor was developed by wet etching and splicing techniques. Small dips in fiber cores can be fabricated by differential etching. By splicing the etched fiber with another fiber, a microgap was generated inside the fiber which functioned as a reflector. Low-finesse FP 
cavities were formed between the microgap reflectors or cleaved fiber end. This process provides another simple and cost-effective method in IFPI sensor fabrication. The multiplexing of microgap sensor with other sensors provides an effective method for temperature-compensated measurement. Multicavity sensors for temperaturecompensated thin-film measurement were constructed by multiplexing microgap sensors. It not only has advantages similar to tubing-based MFPI sensors, but also excels at its simplicity in fabrication process and flexibility in cavity lengths. Furthermore, the temperature sensitivity for temperature compensation has increased by an order of magnitude from the tubing-based MFPI sensor. Thus the thin-film characteristics under temperature variations could be examined more accurately.

Electrostatic self-assembly provides an effective and convenient method for surface modification to retain activities of proteins in fiber optic biosensors. The characteristics of polymer self-assembly were investigated by ellipsometry, absorption spectroscopy and fiber-optic interferometry. Experimental design and analysis were applied to determine the relative effects of the factors. Systematic study of the multiple factors provided the optimum conditions for polymer self-assembly. The long-term stability and low-absorption in NIR range of polymer self-assembly made it suitable as a precursor film in fiber-optic biosensing. In monitoring the adsorption process, the interferometric method demonstrates higher sensitivity than intensity-based measurements used previously. Adsorption of a single monolayer, only nanometers thick, can be monitored by MFPI sensors. The thickness difference existing between the components reveals more detailed information on self-assembly. The high sensitivity of multicavity sensors demonstrates their capability for sensing the immunoreactions at the interfaces.

Proteins can adsorb onto the polymer film by hydrophobic and electrostatic interactions. Comparison of the adsorption of $\operatorname{IgG}$ between positively and negatively charged polymer self-assemblies suggests that hydrophobic interactions may be the dominating factor for our immobilization process. The different thicknesses of the adsorbed layers on both types of films may be a reflection of the intensity of the 
hydrophobic interactions and the coexistence of positively charged and negatively charged proteins in the polyclonal antibodies. The saturating thickness of immobilized $\operatorname{IgG}$ is around the thickness of an $\operatorname{IgG}$ monolayer. The feasibility of using a multicavity FP sensor as a biosensor has been verified. Proteins can keep their biological activity after immobilization onto the polymer self-assembly. The high affinity between $\operatorname{IgG}$ and corresponding anti-IgG assured the high sensitivity but limited the dynamic range in the steady-state measurement. The embedded proteins in multilayer buildups kept their biological activity even with a polyelectrolyte overlay. The demonstrated additive activity for multilayers of antibodies provides the potential to increase the sensitivity. Nonspecific binding was efficiently suppressed by introducing a binding blocker before immunosensing. A theoretical model of the multicavity sensor was developed for in situ application. The kinetic measurement reveals more details of immunoreactions, such as rate constant. Analysis of the real-time binding profile will also provide a fast and effective way of measuring antigen concentration. Furthermore, the binding kinetics provide a larger dynamic range than the steady-state method. The high temperature sensitivity of microgap MFPI sensors provides the opportunity to investigate the biological reactions under temperature variations. This greatly improves the flexibility in sample handling and measurement accuracy for in-situ and in-vivo applications.

\subsection{Suggestions for future research}

Future work is suggested here for diversification in the sensor structure, enhancement in the reliability of sensor fabrication, integration of the sensing system and reduction of cost. An outline of further investigations is described in the following.

The MFPI sensor is applicable not only to the measurement of polyelectrolyte self-assembly but also to the in situ monitoring of the binding process in biosensing applications, including antibody-antigen interactions and DNA hybridization events. Other surface modification and immobilization methods such as covalent attachment, biomolecular interactions and host matrix encapsulation will increase the flexibility of binding different receptors onto the fiber optic sensors. Varying the sensing material 
incorporated into the sensor may expand the application area into gas and chemical sensing.

The sensor has a diameter similar to a single mode fiber which can be further reduced by etching techniques. Different approaches in miniaturization of fiber-optic FP sensors have been investigated in our lab. One method is to expose the fiber core, which act as a miniature FP cavity, by chemical etching. The miniature probe enables analytical measurements of chemicals species in specific locations and limited quantity of analyte.

MFPI sensors are fabricated on single-mode fibers. The sensing area is limited by the mode-field diameter which is around $10 \mu \mathrm{m}$ at $1550 \mathrm{~nm}$. The effective thin-film thickness is the average thickness in the sensing area. The nonuniformity of the thin film will affect the measurement accuracy especially in bacterial sensing where cells of around micrometer size are sparsely immobilized. Multimode MFPI sensors with much larger sensing area may provide the possibility of monitoring bacteria.

The MFPI sensor may be incorporated into the white light interferometric sensor systems developed in our lab. An LED as the low-cost broadband light source and a fiber spectrometer as the detector can provide high stability, high sensitivity, low cost and compact size.

\subsection{Summary of contributions}

This section outlines the major contributions of this dissertation.

- Designed and fabricated fiber optic multicavity Fabry-Perot interferometric (MFPI) sensors including a tubing-based MFPI sensor and a microgap MFPI sensor.

- Proposed and demonstrated built-in temperature compensation for MFPI sensors.

- Demonstrated the polyelectrolyte self-assembly as an effective surface modification method for immobilization of biological molecules onto optical fibers. 
- Optimized polyelectrolyte self-assembly for optical biosensing by experiment design and analysis.

- Demonstrated the application of MFPI sensors as miniature, low-cost and efficient methods in the measurement of adsorption of polymer self-assembly, proteins and immunoreactions.

\subsection{List of related publications}

1. Zhang, Y., K.L. Cooper, and A. Wang, Multicavity Fabry-Perot interferometric thin-film sensor with built-in temperature compensation. IEEE Photon. Tech. L., 2005. 17(12): p. 2712-2714.

2. Zhang, Y., H. Shibru, K.L. Cooper, and A. Wang, Miniature fiber-optic multicavity Fabry-Perot interferometric biosensor. Opt. Lett., 2005. 30(9): p. 1021-1023.

3. Kim, D.W., Y. Zhang, K.L. Cooper, and A. Wang, In-fiber reflection mode interferometer based on a long-period grating for external refractive-index measurement. Appl. Opt., 2005. 44(26): p. 5368-5373.

4. Shibru, H., Y. Zhang, K.L. Cooper, G.R. Pickrell, and A. Wang, Optimization of layer by layer electrostatic self-assembly processing parameters for optical biosensing. Opt. Eng., 2005 (In press).

5. Chen, X., F. Shen, A. Wang, Z. Wang, and Y. Zhang. Novel Fabry-Perot fiber optic sensor with multiple applications. in Sensors for Harsh Environments. 2004. Philadelphia, PA, United States: Proceedings of SPIE - The International Society for Optical Engineering Sensors for Harsh Environments. v 55902004.

6. Chen, X., Y. Zhang, G. Pickrell, and J. Antony, Experimental design in fiber optic sensor development. International Journal of Productivity and Performance Management, 2004. 53(8): p. 713-725. 
7. Han, M., Y. Zhang, F.B. Shen, G.R. Pickrell, and A.B. Wang, Signal-processing algorithm for white-light optical fiber extrinsic Fabry-Perot interferometric sensors. Opt. Lett., 2004. 29(15): p. 1736-1738.

8. Zhang, Y., B. Qi, Y. Duan, Y. Zhang, G. Pickrell, R.G. May, and A. Wang. Single-crystal sapphire high temperature sensor. in Fiber Optic Sensor Technology and Applications 2001. 2001. Newton, MA, United States: Proceedings of SPIE - The International Society for Optical Engineering. v 4578 2001. 


\section{References}

1. Thevenot, D.R., et al., Electrochemical biosensors: recommended definitions and classification. Biosens. Bioelectron., 2001. 16(1-2): p. 121-131.

2. Marazuela, M.D. and M.C. Moreno-Bondi, Fiber-optic biosensors - an overview. Anal. Bioanal. Chem., 2002. 372(5-6): p. 664-682.

3. Maiman, T.H., Optical maser action in ruby. Br. Commun. Electron., 1960. 7(9): p. 674-675.

4. Kao, K.C. and G.A. Hockham, Dielectric-fibre surface waveguides for optical frequencies. Proc. Inst. Electr. Eng., 1966. 113(7): p. 1151-1158.

5. Kapron, F.P., D.B. Keck, and R.D. Maurer, Radiation losses in glass optical waveguides. Appl. Phys. Lett., 1970: p. 423-425.

6. Johnson, C.C., Fiber optic probe for oxygen saturation and dye concentration monitoring. Biomed. Sci. Instrum., 1974. 10: p. 45-49.

7. Lubbers, D.W. and N. Opitz, Po2-Optode, a New Tool to Measure Po2 of Biological Gases and Fluids by Quantitative Fluorescence Photometry. Pflugers Arch., 1975. 359: p. R145-R145.

8. Arnold, M.A., Enzyme-based fiber optic sensor. Anal. Chem., 1985. 57(2): p. 565-566.

9. Fuh, M.R., L.W. Burgess, and G.D. Christian, Single fiber-optic fluorescence enzyme-based sensor. Anal. Chem., 1988. 60(5): p. 433-435.

10. Anderson, F.P. and W.G. Miller, Fiber optic immunochemical sensor for continuous, reversible measurement of phenytoin. Clin. Chem., 1988. 34(7): p. 1417-1421.

11. Cordek, J., X.W. Wang, and W.H. Tan, Direct immobilization of glutamate dehydrogenase on optical fiber probes for ultrasensitive glutamate detection. Anal. Chem., 1999. 71(8): p. 1529-1533.

12. Wolfbeis, O.S., et al., Fluorimetric Analysis .9. A Fast Responding Fluorescence Sensor for Oxygen. Mikrochim. Acta, 1984. 1(1-2): p. 153-158.

13. Peterson, J.I., R.V. Fitzgerald, and D.K. Buckhold, Fiber-Optic Probe for Invivo Measurement of Oxygen Partial-Pressure. Anal. Chem., 1984. 56(1): p. 62-67.

14. Opitz, N., H.J. Graf, and D.W. Lubbers, Oxygen Sensor for the TemperatureRange 300-K to 500-K Based on Fluorescence Quenching of Indicator-Treated Silicone-Rubber Membranes. Sens. Actuators, 1988. 13(2): p. 159-163.

15. Potyrailo, R.A. and G.M. Hieftje, Oxygen detection by fluorescence quenching of tetraphenylporphyrin immobilized in the original cladding of an optical fiber. Anal. Chim. Acta, 1998. 370(1): p. 1-8.

16. Liu, Y.M., et al., Evaluation of Some Immobilized Room-Temperature Phosphorescent Metal-Chelates as Sensing Materials for Oxygen. Anal. Chem., 1994. 66(6): p. 836-840.

17. Mills, A. and M.D. Thomas, Effect of plasticizer viscosity on the sensitivity of an $[R U(b p y)(3)(2+)(P h 4 B-)(2)]$-based optical oxygen sensor. Analyst, 1998. 123(5): p. $1135-1140$. 
18. Xu, W.Y., et al., Oxygen sensors based on luminescence quenching of metal complexes: Osmium complexes suitable for laser diode excitation. Anal. Chem., 1996. 68(15): p. 2605-2609.

19. Wolfbeis, O.S., Fiber optic chemical sensors and biosensors. 1991, Boca Raton, Fla.: CRC Press.

20. Xavier, M.P., et al., Oxygen sensing in nonaqueous media using porous glass with covalently bound luminescent Ru(II) complexes. Anal. Chem., 1998. 70(24): p. 5184-5189.

21. Garcia-Fresnadillo, D., et al., Luminescent nafion membranes dyed with ruthenium(II) complexes as sensing materials for dissolved oxygen. Langmuir, 1999. 15(19): p. 6451-6459.

22. Rosenzweig, Z. and R. Kopelman, Analytical properties of miniaturized oxygen and glucose fiber optic sensors. Sens. Actuators B Chem., 1996. 36(1-3): p. 475483.

23. Li, X.P. and Z. Rosenzweig, A fiber optic sensor for rapid analysis of bilirubin in serum. Anal. Chim. Acta, 1997. 353(2-3): p. 263-273.

24. Marazuela, M.D., et al., Free cholesterol fiber-optic biosensor for serum samples with simplex optimization. Biosens. Bioelectron., 1997. 12(3): p. 233-240.

25. Muller, C., et al., Optical chemo- and biosensors for use in clinical applications. Sens. Actuators B Chem., 1997. 40(1): p. 71-77.

26. Kuswandi, B., Simple optical fibre biosensor based on immobilised enzyme for monitoring of trace heavy metal ions. Anal. Bioanal. Chem., 2003. 376(7): p. 1104-1110.

27. Andreou, V.G. and Y.D. Clonis, Novel fiber-optic biosensor based on immobilized glutathione S-transferase and sol-gel entrapped bromcresol green for the determination of atrazine. Anal. Chim. Acta, 2002. 460(2): p. 151-161.

28. Zhang, W., H.D. Chang, and G.A. Rechnitz, Dual-enzyme fiber optic biosensor for pyruvate. Anal. Chim. Acta, 1997. 350(1-2): p. 59-65.

29. Michel, P.E., S.M. Gautier-Sauvigne, and L.J. Blum, A transient enzymatic inhibition as an efficient tool for the discriminating bioluminescent analysis of three adenylic nucleotides with a fiberoptic sensor based on a compartmentalized tri-enzymatic sensing layer. Anal. Chim. Acta, 1998. 360(1-3): p. 89-99.

30. Anderson, G.P. and N.L. Nerurkar, Improved fluoroimmunoassays using the dye Alexa Fluor 647 with the RAPTOR, a fiber optic biosensor. J. Immunol. Methods, 2002. 271(1-2): p. 17-24.

31. Brecht, A., et al., A Direct Optical Immunosensor for Atrazine Detection. Anal. Chim. Acta, 1995. 311(3): p. 289-299.

32. Hook, F., et al., A comparative study of protein adsorption on titanium oxide surfaces using in situ ellipsometry, optical waveguide lightmode spectroscopy, and quartz crystal microbalance/dissipation. Colloids Surf. B, 2002. 24(2): p. 155-170.

33. Skladal, P., A.P. Deng, and V. Kolar, Resonant mirror-based optical immunosensor: application for the measurement of atrazine in soil. Anal. Chim. Acta, 1999. 399(1-2): p. 29-36. 
34. Oh, B.K., et al., Surface plasmon resonance immunosensor for the detection of Yersinia enterocolitica. Colloids Surf. A, 2005. 257-58: p. 369-374.

35. Zhang, Y., et al., Miniature fiber-optic multicavity Fabry-Perot interferometric biosensor. Opt. Lett., 2005. 30(9): p. 1021-1023.

36. Bakaltcheva, I.B., et al., Multi-analyte explosive detection using a fiber optic biosensor. Anal. Chim. Acta, 1999. 399(1-2): p. 13-20.

37. Toppozada, A.R., et al., Evaluation of a fiber optic immunosensor for quantitating cocaine in coca leaf extracts. Biosens. Bioelectron., 1997. 12(2): p. 113-124.

38. Nath, N., et al., A rapid reusable fiber optic biosensor for detecting cocaine metabolites in urine. J. Anal. Toxicol., 1999. 23(6): p. 460-467.

39. Mouvet, C., et al., Determination of simazine in water samples by waveguide surface plasmon resonance. Anal. Chim. Acta, 1997. 338(1-2): p. 109-117.

40. Brecht, A., et al., Optical immunoprobe development for multiresidue monitoring in water. Anal. Chim. Acta, 1998. 362(1): p. 69-79.

41. Kwon, H.J., H.I. Balcer, and K.A. Kang, Sensing performance of protein C immuno-biosensor for biological samples and sensor minimization. Comp.

Biochem. Physiol. A. Mol. Integr. Physiol., 2002. 132(1): p. 231-238.

42. King, K.D., et al., Detecting staphylococcal enterotoxin B using an automated fiber optic biosensor. Biosens. Bioelectron., 1999. 14(2): p. 163-170.

43. Zhou, C.H., et al., A compact fiber-optic immunosensor for Salmonella based on evanescent wave excitation. Sens. Actuators B Chem., 1997. 42(3): p. 169-175.

44. Lu, B., C.L. Lu, and Y. Wei, A Planar Quartz Wave-Guide Immunosensor Based on Tirf Principle. Anal. Lett., 1992. 25(1): p. 1-10.

45. Wijesuriya, D., et al., Regeneration of immobilized antibodies on fiber optic probes. Biosens. Bioelectron., 1994. 9(8): p. 585-592.

46. Barker, S.L.R., et al., Fiber-optic nitric oxide-selective biosensors and nanosensors. Anal. Chem., 1998. 70(5): p. 971-976.

47. Barker, S.L.R., et al., Cellular applications of a sensitive and selective fiber optic nitric oxide biosensor based on a dye-labeled heme domain of soluble guanylate cyclase. Anal. Chem., 1999. 71(11): p. 2071-2075.

48. Almadidy, A., et al., Direct selective detection of genomic DNA from coliform using a fiber optic biosensor. Anal. Chim. Acta, 2002. 461(1): p. 37-47.

49. Almadidy, A., et al., A fibre-optic biosensor for detection of microbial contamination. Can. J. Chem., 2003. 81(5): p. 339-349.

50. Wang, X.F. and U.J. Krull, Tethered thiazole orange intercalating dye for development of fibre-optic nucleic acid biosensors. Anal. Chim. Acta, 2002. 470(1): p. 57-70.

51. Watterson, J.H., et al., Influences of non-selective interactions of nucleic acids on response rates of nucleic acid fiber optic biosensors. Fresenius. J. Anal. Chem., 2001. 369(7-8): p. 601-608.

52. Abel, A.P., et al., Fiber-optic evanescent wave biosensor for the detection of oligonucleotides. Anal. Chem., 1996. 68(17): p. 2905-2912.

53. Schmidt, P.M., et al., Detection of activity of telomerase in tumor cells using fiber optical biosensors. Biosens. Bioelectron., 2002. 17(11-12): p. 1081-1087. 
54. Kleinjung, F., et al., Fibre-optic genosensor for specific determination of femtomolar DNA oligomers. Anal. Chim. Acta, 1997. 350(1-2): p. 51-58.

55. Ferguson, J.A., et al., A fiber-optic DNA biosensor microarray for the analysis of gene expression. Nat. Biotechnol., 1996. 14(13): p. 1681-1684.

56. Ignatov, S.G., J.A. Ferguson, and D.R. Walt, A fiber-optic lactate sensor based on bacterial cytoplasmic membranes. Biosens. Bioelectron., 2001. 16(1-2): p. 109113.

57. Frense, D., A. Muller, and D. Beckmann, Detection of environmental pollutants using optical biosensor with immobilized algae cells. Sens. Actuators B Chem., 1998. 51(1-3): p. 256-260.

58. Corbisier, P., et al., Whole cell-and protein-based biosensors for the detection of bioavailable heavy metals in environmental samples. Anal. Chim. Acta, 1999. 387(3): p. 235-244.

59. Roda, A., et al., Sensitive determination of urinary mercury(II) by a bioluminescent transgenic bacteria-based biosensor. Anal. Lett., 2001. 34(1): p. 29-41.

60. Ripp, S., et al., Controlled field release of a bioluminescent genetically engineered microorganism for bioremediation process monitoring and control. Environ. Sci. Technol., 2000. 34(5): p. 846-853.

61. Mulchandani, A., I. Kaneva, and W. Chen, Biosensor for direct determination of organophosphate nerve agents using recombinant Escherichia coli with surfaceexpressed organophosphorus hydrolase. 2. Fiber optic microbial biosensor. Anal. Chem., 1998. 70(23): p. 5042-5046.

62. Choi, S.H. and M.B. Gu, A portable toxicity biosensor using freeze-dried recombinant bioluminescent bacteria. Biosens. Bioelectron., 2002. 17(5): p. 433440.

63. Choi, J.W., et al., Optical organophosphorus biosensor consisting of acetylcholinesterase/viologen hetero Langmuir-Blodgett film. Biosens. Bioelectron., 2001. 16(9-12): p. 937-943.

64. Sotomayor, M.D.T., et al., Bi-enzymatic optode detection system for oxalate determination based on a natural source of enzyme. Anal. Chim. Acta, 2001. 447(1-2): p. 33-40.

65. Paranjpe, P., et al., A disposable optrode using immobilized tyrosinase films. Anal. Biochem., 2001. 294(2): p. 102-107.

66. Watanabe, M. and K. Kajikawa, An optical fiber biosensor based on anomalous reflection of gold. Sens. Actuators B Chem., 2003. 89(1-2): p. 126-130.

67. Cunningham, B., et al., Colorimetric resonant reflection as a direct biochemical assay technique. Sens. Actuators B Chem., 2002. 81(2-3): p. 316-328.

68. Doong, R.A. and H.C. Tsai, Immobilization and characterization of sol-gelencapsulated acetylcholinesterase fiber-optic biosensor. Anal. Chim. Acta, 2001. 434(2): p. 239-246.

69. Issberner, J.P., et al., Combined imaging and chemical sensing of L-glutamate release from the foregut plexus of the lepidopteran, Manduca sexta. J. Neurosci. Methods, 2002. 120(1): p. 1-10. 
70. Gerion, D., et al., Room-temperature single-nucleotide polymorphism and multiallele DNA detection using fluorescent nanocrystals and microarrays. Anal. Chem., 2003. 75(18): p. 4766-4772.

71. Medintz, I.L., et al., Self-assembled nanoscale biosensors based on quantum dot FRET donors. Nat. Mater., 2003. 2(9): p. 630-638.

72. Robelek, R., et al., Multiplexed hybridization detection of quantum dotconjugated DNA sequences using surface plasmon enhanced fluorescence microscopy and spectrometry. Anal. Chem., 2004. 76(20): p. 6160-6165.

73. Medintz, I.L., et al., Quantum dot bioconjugates for imaging, labelling and sensing. Nat. Mater., 2005. 4(6): p. 435-446.

74. Fahnrich, K.A., M. Pravda, and G.G. Guilbault, Recent applications of electrogenerated chemiluminescence in chemical analysis. Talanta, 2001. 54(4): p. 531-559.

75. Ramos, M.C., M.C. Torijas, and A.N. Diaz, Enhanced chemiluminescence biosensor for the determination of phenolic compounds and hydrogen peroxide. Sens. Actuators B Chem., 2001. 73(1): p. 71-75.

76. Zhu, L.D., et al., Electrochemiluminescent determination of glucose with a sol-gel derived ceramic-carbon composite electrode as a renewable optical fiber biosensor. Sens. Actuators B Chem., 2002. 84(2-3): p. 265-270.

77. Marquette, C.A., B.D. Leca, and L.J. Blum, Electrogenerated chemiluminescence of luminol for oxidase-based fibre-optic biosensors. Luminescence, 2001. 16(2): p. 159-165.

78. Liedberg, B., C. Nylander, and I. Lundstrom, Biosensing with Surface-Plasmon Resonance - How It All Started. Biosens. Bioelectron., 1995. 10(8): p. R1-R9.

79. Slavik, R., J. Homola, and J. Ctyroky, Single-mode optical fiber surface plasmon resonance sensor. Sens. Actuators B Chem., 1999. 54(1-2): p. 74-79.

80. Kurihara, K., et al., Fiber-optic conical microsensors for surface plasmon resonance using chemically etched single-mode fiber. Anal. Chim. Acta, 2004. 523(2): p. 165-170.

81. Iga, M., A. Seki, and K. Watanabe, Hetero-core structured fiber optic surface plasmon resonance sensor with silver film. Sens. Actuators B Chem., 2004. 101(3): p. 368-372.

82. Obando, L.L. and K.S. Booksh, Tuning dynamic range and sensitivity of whitelight, multimode, fiber-optic surface plasmon resonance sensors. Anal. Chem., 1999. 71(22): p. 5116-5122.

83. Underwood, S. and P. Mulvaney, Effect of the Solution Refractive-Index on the Color of Gold Colloids. Langmuir, 1994. 10(10): p. 3427-3430.

84. Cheng, S.F. and L.K. Chau, Colloidal gold-modified optical fiber for chemical and biochemical sensing. Anal. Chem., 2003. 75(1): p. 16-21.

85. Mitsui, K., Y. Handa, and K. Kajikawa, Optical fiber affinity biosensor based on localized surface plasmon resonance. Appl. Phys. Lett., 2004. 85(18): p. 42314233. 
86. Gessner, R., et al., Raman spectroscopy investigation of biological materials by use of etched and silver coated glass fiber tips. Biopolymers, 2002. 67(4-5): p. 327-330.

87. Stokes, D.L., Z. Chi, and T. Vo-Dinh, Surface-enhanced-Raman-scatteringinducing nanoprobe for spectrochemical analysis. Appl. Spectrosc., 2004. 58(3): p. 292-298.

88. Epstein, J.R., M. Lee, and D.R. Walt, High-density fiber-optic genosensor microsphere array capable of zeptomole detection limits. Anal. Chem., 2002. 74(8): p. 1836-1840.

89. Biran, I. and D.R. Walt, Optical imaging fiber-based single live cell arrays: a high-density cell assay platform. Anal. Chem., 2002. 74(13): p. 3046-3054.

90. Kersey, A.D., et al., Fiber grating sensors. J. Lightwave Technol., 1997. 15(8): p. 1442-1463.

91. Iadicicco, A., et al., Thinned fiber Bragg gratings as high sensitivity refractive index sensor. IEEE Photon. Tech. L., 2004. 16(4): p. 1149-1151.

92. Iadicicco, A., et al., Nonuniform thinned fiber Bragg gratings for simultaneous refractive index and temperature measurements. IEEE Photon. Tech. L., 2005. 17(7): p. 1495-1497.

93. Bhatia, V., Applications of long-period gratings to single and multi-parameter sensing. Opt. Express, 1999. 4(11): p. 457-466.

94. DeLisa, M.P., et al., Evanescent wave long-period fiber bragg grating as an immobilized antibody biosensor. Anal. Chem., 2000. 72(13): p. 2895-2900.

95. Weisser, M., et al., Specific bio-recognition reactions observed with an integrated Mach-Zehnder interferometer. Biosens. Bioelectron., 1999. 14(4): p. 405-411.

96. Busse, S., et al., Gold and thiol surface functionalized integrated optical MachZehnder interferometer for sensing purposes. Sens. Actuators B Chem., 1999. 60(2-3): p. 148-154.

97. Choquette, S.J. and L. Locasciobrown, Thermal Detection of Enzyme-Labeled Antigen-Antibody Complexes Using Fiberoptic Interferometry. Sens. Actuators B Chem., 1994. 22(2): p. 89-96.

98. Lin, V.S.Y., et al., A porous silicon-based optical interferometric biosensor. Science, 1997. 278(5339): p. 840-843.

99. O, B.H., et al., Vapor sensor realized in an ultracompact polarization interferometer built of a freestanding porous-silicon form birefringent film. IEEE Photon. Tech. L., 2003. 15(6): p. 834-836.

100. Starodub, V.M., et al., Control of myoglobin level in a solution by an immune sensor based on the photoluminescence of porous silicon. Sens. Actuators B Chem., 1999. 58(1-3): p. 409-414.

101. Chan, S., et al., Nanoscale silicon microcavities for biosensing. Mater. Sci. Eng., C, 2001. 15(1-2): p. 277-282.

102. Galeazzo, E., et al., Gas sensitive porous silicon devices: responses to organic vapors. Sens. Actuators B Chem., 2003. 93(1-3): p. 384-390. 
103. Dancil, K.P.S., D.P. Greiner, and M.J. Sailor, A porous silicon optical biosensor: Detection of reversible binding of IgG to a protein A-modified surface. J. Am. Chem. Soc., 1999. 121(34): p. 7925-7930.

104. Gauglitz, G., et al., Chemical and Biochemical Sensors Based on Interferometry at Thin (Multi-)Layers. Sens. Actuators B Chem., 1993. 11(1-3): p. 21-27.

105. Arregui, F.J., et al., Optical fiber nanometer-scale Fabry-Perot interferometer formed by the ionic self-assembly monolayer process. Opt. Lett., 1999. 24(9): p. 596-598.

106. Arregui, F.J., et al., Optical fiber humidity sensor using a nano Fabry-Perot cavity formed by the ionic self-assembly method. Sens. Actuators B Chem., 1999. 59(1): p. 54-59.

107. Del Villar, I., et al., ESA-based in-fiber nanocavity for hydrogen-peroxide detection. IEEE Trans. Nanotech., 2005. 4(2): p. 187-193.

108. Lin, C.-J., et al. A novel in-vitro and in-situ immunoassay biosensor based on fiber-optic Fabry-Perot interferometry. in Second European Workshop on Optical Fibre Sensors, EWOFS'04. 2004. Santander, Spain: Proceedings of SPIE - The International Society for Optical Engineering Second European Workshop on Optical Fibre Sensors, EWOFS'04. v 55022004.

109. Zhujun, Z., et al., Polyvinyl-Alcohol) as a Substrate for Indicator Immobilization for Fiber-Optic Chemical Sensors. Anal. Chem., 1989. 61(3): p. 202-205.

110. Chen, Z., et al., Molecular assembly of multilayer enzyme: toward the development of a chemiluminescence-based fiber optic biosensor. Mater. Sci. Eng., C, 1996. 4(3): p. 155-159.

111. Luo, S.F. and D.R. Walt, Avidin Biotin Coupling as a General-Method for Preparing Enzyme-Based Fiber-Optic Sensors. Anal. Chem., 1989. 61(10): p. 1069-1072.

112. Dave, B.C., et al., Sol-Gel Encapsulation Methods for Biosensors. Anal. Chem., 1994. 66(22): p. A1120-a1127.

113. Gill, I. and A. Ballesteros, Bioencapsulation within synthetic polymers (Part 1): sol-gel encapsulated biologicals. Trends Biotechnol., 2000. 18(7): p. 282-296.

114. Diaz, A.N. and M.C.R. Peinado, Sol-gel cholinesterase biosensor for organophosphorus pesticide fluorimetric analysis. Sens. Actuators B Chem., 1997. 39(1-3): p. 426-431.

115. Gill, I. and A. Ballesteros, Bioencapsulation within synthetic polymers (Part 2): non-sol-gel protein-polymer biocomposites. Trends Biotechnol., 2000. 18(11): p. 469-479.

116. Bhatia, S.K., et al., Use of Thiol-Terminal Silanes and Heterobifunctional Crosslinkers for Immobilization of Antibodies on Silica Surfaces. Anal. Biochem., 1989. 178(2): p. 408-413.

117. Bakaltcheva, I.B., L.C. Shriver-Lake, and F.S. Ligler, Fiber optic biosensor for multianalyte detection: importance of preventing fluorophore aggregation. Sens. Actuators B Chem., 1998(1-3): p. 46-51.

118. Brummel, K.E., J. Wright, and M.E. Eldefrawi, Fiber optic biosensor for cyclodiene insecticides. J. Agric. Food Chem., 1997. 45(8): p. 3292-3298. 
119. Cullum, B.M., et al., Intracellular measurements in mammary carcinoma cells using fiber-optic nanosensors. Anal. Biochem., 2000. 277(1): p. 25-32.

120. Mosiello, L., et al., A fibre-optic immunosensor for 2,4-dichlorophenoxyacetic acid detection. Sens. Actuators B Chem., 1997. 39(1-3): p. 353-359.

121. Rowe, C.A., et al., Rapid defection of D-dimer using a fiber optic biosensor. Thromb. Haemostasis, 1998. 79(1): p. 94-98.

122. Marks, R.S., et al., An innovative strategy for immobilization of receptor proteins on to an optical fiber by use of poly(pyrrole-biotin). Anal. Bioanal. Chem., 2002. 374(6): p. 1056-1063.

123. Grant, S.A. and R.S. Glass, Sol-gel-based biosensor for use in stroke treatment. IEEE Trans. Biomed. Eng., 1999. 46(10): p. 1207-1211.

124. HidalgoAlvarez, R. and F. GalisteoGonzalez, The adsorption characteristics of immunoglobulins. Heterogen. Chem. Rev., 1995. 2(4): p. 249-268.

125. Buijs, J., et al., Adsorption of Monoclonal Iggs and Their F(Ab')(2) Fragments onto Polymeric Surfaces. Colloids Surf. B, 1995. 5(1-2): p. 11-23.

126. Buijs, J., et al., Adsorption dynamics of $\operatorname{Ig} G$ and its $F\left(a b^{\prime}\right)(2)$ and $F c$ fragments studied by reflectometry. J. Colloid Interface Sci., 1996. 178(2): p. 594-605.

127. Harlow, E. and D. Lane, Using antibodies: a laboratory manual. 1999, Cold Spring Harbor, N.Y.: Cold Spring Harbor Laboratory Press.

128. Vijayendran, R.A. and D.E. Leckband, A quantitative assessment of heterogeneity for surface-immobilized proteins. Anal. Chem., 2001. 73(3): p. 471-480.

129. Lu, B., M.R. Smyth, and R. OKennedy, Oriented immobilization of antibodies and its applications in immunoassays and immunosensors. Analyst, 1996. 121(3): p. R29-R32.

130. Tominaga, J., et al., An enzymatic strategy for site-specific immobilization of functional proteins using microbial transglutaminase. Enzyme Microb. Technol., 2004. 35(6-7): p. 613-618.

131. Iler, R.K., The chemistry of silica: solubility, polymerization, colloid and surface properties, and biochemistry. 1979, New York: Wiley.

132. Berg, J.M., J.L. Tymoczko, and L. Stryer, Biochemistry. 5th ed. 2002, New York: W.H. Freeman.

133. Bae, T., et al., Interferometric fiber-optic sensor embedded in a spark plug for incylinder pressure measurement in engines. Appl. Opt., 2003. 42(6): p. 1003-1007.

134. Grossmann, B.G. and L.T. Huang, Fiber optic sensor array for multi-dimensional strain measurement. Smart Mater. Struct., 1998. 7(2): p. 159-165.

135. Zhu, Y.Z. and A.B. Wang, Miniature fiber-optic pressure sensor. IEEE Photon. Tech. L., 2005. 17(2): p. 447-449.

136. Qi, B., et al. Fiber optic pressure and temperature sensors for oil down hole application. in Fiber Optic Sensor Technology and Applications 2001. 2001. Newton, MA, United States: Proceedings of SPIE - The International Society for Optical Engineering. v 45782001.

137. Bhatia, V., et al., Multiple strain state measurements using conventional and absolute optical fiber-based extrinsic Fabry-Perot interferometric strain sensors. Smart Mater. Struct., 1995. 4(4): p. 240-245. 
138. Sirkis, J., et al., In-Line Fiber Etalon (Ilfe) Fiberoptic Strain Sensors. J. Lightwave Technol., 1995. 13(7): p. 1256-1263.

139. Chen, X., et al. Novel Fabry-Perot fiber optic sensor with multiple applications. in Sensors for Harsh Environments. 2004. Philadelphia, PA, United States: Proceedings of SPIE - The International Society for Optical Engineering Sensors for Harsh Environments. v 55902004.

140. Peng, W., et al., Self-compensating fiber optic flow sensor system and its field applications. Appl. Opt., 2004. 43(8): p. 1752-1760.

141. Wang, X., et al. Verifying an all fused silica miniature optical fiber tip pressure sensor performance with turbine engine field test. in Sensors for Harsh Environments II. 2005. Boston, MA, United States: Proceedings of SPIE - The International Society for Optical Engineering Sensors for Harsh Environments. V 59982005.

142. Qi, B., et al., Novel data processing techniques for dispersive white light interferometer. Opt. Eng., 2003. 42(11): p. 3165-3171.

143. Lee, C.E. and H.F. Taylor, Interferometric Optical Fiber Sensors Using Internal Mirrors. Electron. Lett., 1988. 24(4): p. 193-194.

144. Betts, P. and J.A. Davis, Bragg grating Fabry-Perot interferometer with variable finesse. Opt. Eng., 2004. 43(5): p. 1258-1259.

145. Shen, F., et al. UV-induced intrinsic Fabry-Perot interferometric fiber sensors. in Sensors for Harsh Environments. 2004. Philadelphia, PA, United States:

Proceedings of SPIE - The International Society for Optical Engineering Sensors for Harsh Environments. v 55902004.

146. Wang, X., et al. An optical fiber tip pressure sensor for medical applications. in 2005 Quantum Electronics and Laser Science Conference (QELS). 2005.

Baltimore, MD, United States: Quantum Electronics and Laser Science Conference (QELS). v 22005.

147. Han, M., et al., Signal-processing algorithm for white-light optical fiber extrinsic Fabry-Perot interferometric sensors. Opt. Lett., 2004. 29(15): p. 1736-1738.

148. Nemoto, S. and T. Makimoto, Analysis of Splice Loss in Single-Mode Fibers Using a Gaussian Field Approximation. Opt. Quantum Electron., 1979. 11(5): p. 447-457.

149. Marcuse, D., Loss Analysis of Single-Mode Fiber Splices. Bell Sys. Tech. J., 1977. 56(5): p. 703-718.

150. Xu, J.C., et al., A novel temperature-insensitive optical fiber pressure sensor for harsh environments. IEEE Photon. Tech. L., 2005. 17(4): p. 870-872.

151. Klein, M.V. and T.E. Furtak, Optics. 2nd ed. 1986, New York: Wiley.

152. Ma, J.J., W.J. Bock, and W. Urbanczyk, Error analysis of temperaturecompensated white-light interferometric fiber-optic strain sensor. Sens. Actuators A Phys., 2004. 112(1): p. 25-31.

153. van Brakel, A. and P.L. Swart, Temperature-compensated optical fiber Michelson refractometer. Opt. Eng., 2005. 44(2): p. 0205041-0205042. 
154. Zhang, Y., K.L. Cooper, and A. Wang, Multicavity Fabry-Perot interferometric thin-film sensor with built-in temperature compensation. IEEE Photon. Tech. L., 2005. 17(12): p. 2712-2714.

155. Lee, C.E., R.A. Atkins, and H.F. Taylor, Performance of a Fiber-Optic Temperature Sensor from -200-Degrees-C to 1050-Degrees-C. Opt. Lett., 1988. 13(11): p. 1038-1040.

156. Weber, M.J., Handbook of optical materials. CRC Press laser and optical science and technology series. 2003, Boca Raton, Fla.: CRC Press.

157. Touloukian, Y.S., Thermal expansion-nonmetallic solids. Thermophysical properties of matter; v. 13. 1977, New York: IFI/Plenum.

158. Lvov, Y., G. Decher, and H. Mohwald, Assembly, Structural Characterization, and Thermal-Behavior of Layer-by-Layer Deposited Ultrathin Films of Poly(Vinyl Sulfate) and Poly(Allylamine). Langmuir, 1993. 9(2): p. 481-486.

159. Lee, C.E., et al., In-Line Fiber Fabry-Perot-Interferometer with High-Reflectance Internal Mirrors. J. Lightwave Technol., 1992. 10(10): p. 1376-1379.

160. von Lerber, T. and M.W. Sigrist, Cavity-ring-down principle for fiber-optic resonators: experimental realization of bending loss and evanescent-field sensing. Appl. Opt., 2002. 41(18): p. 3567-3575.

161. Gupta, M., H. Jiao, and A. O'Keefe, Cavity-enhanced spectroscopy in optical fibers. Opt. Lett., 2002. 27(21): p. 1878-1880.

162. Slavik, R., S. Doucet, and S. LaRochelle, High-performahce all-fiber Fabry-Perot filters with superimposed chirped Bragg gratings. J. Lightwave Technol., 2003. 21(4): p. 1059-1065.

163. Peng, X.D. and C. Roychoudhuri, Design of high finesse, wideband Fabry-Perot filter based on chirped fiber Bragg grating by numerical method. Opt. Eng., 2000. 39(7): p. 1858-1862.

164. Tsai, W.H. and C.J. Lin, A novel structure for the intrinsic Fabry-Perot fiberoptic temperature sensor. J. Lightwave Technol., 2001. 19(5): p. 682-686.

165. Hoffmann, P., B. Dutoit, and R.P. Salathe, Comparison of mechanically drawn and protection layer chemically etched optical fiber tips. Ultramicroscopy, 1995. 61(1-4): p. 165-170.

166. Stockle, R., et al., High-quality near-field optical probes by tube etching. Appl. Phys. Lett., 1999. 75(2): p. 160-162.

167. Puygranier, B.A.F. and P. Dawson, Chemical etching of optical fibre tips experiment and model. Ultramicroscopy, 2000. 85(4): p. 235-248.

168. Pangaribuan, T., et al., Reproducible Fabrication Technique of Nanometric Tip Diameter Fiber Probe for Photon Scanning Tunneling Microscope. Jpn. J. Appl. Phys. Part 2, 1992. 31(9A): p. L1302-L1304.

169. Ohtsu, M., Progress of High-Resolution Photon Scanning-Tunneling-Microscopy Due to a Nanometric Fiber Probe. J. Lightwave Technol., 1995. 13(7): p. 12001221.

170. Klini, A., et al., Reproducible optical fiber tips for photon scanning tunneling microscopy with very small ( $<5$ degrees) cone angle. J. Lightwave Technol., 1998. 16(7): p. 1220-1227. 
171. Shen, F.B. and A.B. Wang, Frequency-estimation-based signal-processing algorithm for white-light optical fiber Fabry-Perot interferometers. Appl. Opt., 2005. 44(25): p. 5206-5214.

172. Cavicchi, T.J., Digital signal processing. 2000, New York: J. Wiley.

173. Tronin, A., et al., Optimisation of IgG Langmuir film deposition for application as sensing elements. Sens. Actuators B Chem., 1996. 34(1-3): p. 276-282.

174. Lvov, Y., et al., Assembly of Multicomponent Protein Films by Means of Electrostatic Layer-by-Layer Adsorption. J. Am. Chem. Soc., 1995. 117(22): p. 6117-6123.

175. Caruso, F., et al., Assembly of alternating polyelectrolyte and protein multilayer films for immunosensing .2. Langmuir, 1997. 13(13): p. 3427-3433.

176. Decher, G., J.D. Hong, and J. Schmitt, Buildup of Ultrathin Multilayer Films by a Self-Assembly Process .3. Consecutively Alternating Adsorption of Anionic and Cationic Polyelectrolytes on Charged Surfaces. Thin Solid Films, 1992. 210(1-2): p. 831-835.

177. Hammond, P.T., Recent explorations in electrostatic multilayer thin film assembly. Curr. Opin. Colloid In., 1999. 4(6): p. 430-442.

178. Decher, G. and J. Schmitt, Fine-tuning of the film thickness of ultrathin multilayer films composed of consecutively alternating layers of anionic and cationic polyelectrolytes. Prog. Colloid Polym. Sci., 1992. 89(Trends Colloid Interface Sci. VI): p. 160-164.

179. Decher, G. and J.B. Schlenoff, Multilayer thin films: sequential assembly of nanocomposite materials. 2003, Weinheim Cambridge: Wiley-VCH.

180. Losche, M., et al., Detailed structure of molecularly thin polyelectrolyte multilayer films on solid substrates as revealed by neutron reflectometry. Macromolecules, 1998. 31(25): p. 8893-8906.

181. Hao, E.C. and T.Q. Lian, Buildup of polymer/Au nanoparticle multilayer thin films based on hydrogen bonding. Chem. Mater., 2000. 12(11): p. 3392-3396.

182. Fang, M.M., et al., A "mix and match" ionic-covalent strategy for self-assembly of inorganic multilayer films. Abstr. Pap. Amer. Chem. Soc., 1997. 214: p. 45-Mtls.

183. Decher, G., et al., New Nanocomposite Films for Biosensors - Layer-by-Layer Adsorbed Films of Polyelectrolytes, Proteins or DNA. Biosens. Bioelectron., 1994. 9(9-10): p. 677-684.

184. Decher, G. and J.D. Hong, Buildup of Ultrathin Multilayer Films by a SelfAssembly Process .1. Consecutive Adsorption of Anionic and Cationic Bipolar Amphiphiles on Charged Surfaces. Makromolekulare Chemie-Macromolecular Symposia, 1991. 46: p. 321-327.

185. Hong, J.D., et al., Layer-by-layer deposited multilayer assemblies of polyelectrolytes and proteins: From ultrathin films to protein arrays. Prog. Colloid Polym. Sci., 1993. 93(TRENDS IN COLLOID AN): p. 98-102.

186. Lvov, Y., G. Decher, and G. Sukhorukov, Assembly of Thin-Films by Means of Successive Deposition of Alternate Layers of DNA and Poly(Allylamine). Macromolecules, 1993. 26(20): p. 5396-5399. 
187. Cassier, T., K. Lowack, and G. Decher, Layer-by-layer assembled protein/polymer hybrid films: nanoconstruction via specific recognition. Supramol. Sci., 1998. 5(3-4): p. 309-315.

188. Halthur, T.J., P.M. Claesson, and U.M. Elofsson, Stability of polypeptide multilayers as studied by in situ ellipsometry: Effects of drying and post-buildup changes in temperature and $\mathrm{pH}$. J. Am. Chem. Soc., 2004. 126(51): p. 1700917015.

189. Lowman, G.M. and S.K. Buratto, Nanoscale morphology of polyelectrolyte selfassembled films probed by scanning force and near-field scanning optical microscopy. Thin Solid Films, 2002. 405(1-2): p. 135-140.

190. Lavalle, P., et al., Comparison of the structure of polyelectrolyte multilayer films exhibiting a linear and an exponential growth regime: An in situ atomic force microscopy study. Macromolecules, 2002. 35(11): p. 4458-4465.

191. Advincula, R., et al., In situ investigations of polymer self-assembly solution adsorption by surface plasmon spectroscopy. Langmuir, 1996. 12(15): p. 35363540.

192. Ladam, G., et al., In situ determination of the structural properties of initially deposited polyelectrolyte multilayers. Langmuir, 2000. 16(3): p. 1249-1255.

193. Yoo, D., S.S. Shiratori, and M.F. Rubner, Controlling bilayer composition and surface wettability of sequentially adsorbed multilayers of weak polyelectrolytes. Macromolecules, 1998. 31(13): p. 4309-4318.

194. Arregui, F.J., et al., Fabrication of microgratings on the ends of standard optical fibers by the electrostatic self-assembly monolayer process. Opt. Lett., 2001. 26(3): p. 131-133.

195. Ramsden, J.J., Y.M. Lvov, and G. Decher, Determination of Optical Constants of Molecular Films Assembled Via Alternate Polyion Adsorption. Thin Solid Films, 1995. 254(1-2): p. 246-251.

196. Giacomelli, C.E., Adsorption of immunoglobulins at solid-liquid interfaces, in Encyclopedia of Surface and Colloid Science, A.T. Hubbard, Editor. 2002, Marcel Dekker: New York. p. 418-439.

197. Ladam, G., et al., Protein interactions with polyelectrolyte multilayers: Interactions between human serum albumin and polystyrene sulfonate/polyallylamine multilayers. Biomacromolecules, 2000. 1(4): p. 674-687.

198. Ladam, G., et al., Protein adsorption onto auto-assembled polyelectrolyte films. Langmuir, 2001. 17(3): p. 878-882.

199. Marsh, R.J., R.A.L. Jones, and M. Sferrazza, Adsorption and displacement of a globular protein on hydrophilic and hydrophobic surfaces. Colloids Surf. B, 2002. 23(1): p. 31-42.

200. de Jongh, H.H.J., et al., Protein adsorption at air-water interfaces: A combination of details. Biopolymers, 2004. 74(1-2): p. 131-135.

201. Su, T.J., et al., The conformational structure of bovine serum albumin layers adsorbed at the silica-water interface. J. Phys. Chem. B, 1998. 102(41): p. 81008108. 
202. Oshannessy, D.J., et al., Determination of Rate and Equilibrium Binding Constants for Macromolecular Interactions Using Surface-Plasmon Resonance Use of Nonlinear Least-Squares Analysis-Methods. Anal. Biochem., 1993. 212(2): p. 457-468.

203. Jonsson, U., M. Malmqvist, and I. Ronnberg, Adsorption of Immunoglobulin-G, Protein-a, and Fibronectin in the Submonolayer Region Evaluated by a Combined Study of Ellipsometry and Radiotracer Techniques. J. Colloid Interface Sci., 1985. 103(2): p. 360-372.

204. Mondon, M., S. Berger, and C. Ziegler, Scanning-force techniques to monitor time-dependent changes in topography and adhesion force of proteins on surfaces. Anal. Bioanal. Chem., 2003. 375(7): p. 849-855.

205. Lang, W. and R. Zander, Physiological HEPES buffer proposed as a calibrator for pH measurement in human blood. Clin. Chem. Lab. Med., 1999. 37(5): p. 563-571.

206. Rovira-Bru, M., F. Giralt, and Y. Cohen, Protein adsorption onto zirconia modified with terminally grafted polyvinylpyrrolidone. J. Colloid Interface Sci., 2001. 235(1): p. 70-79.

207. Geddes, N.J., et al., Immobilization of Igg onto Gold Surfaces and Its Interaction with Anti-Igg Studied by Surface-Plasmon Resonance. J. Immunol. Methods, 1994. 175(2): p. 149-160.

208. Defeijter, J.A., J. Benjamins, and F.A. Veer, Ellipsometry as a Tool to Study the Adsorption Behavior of Synthetic and Biopolymers at the Air-Water Interface. Biopolymers, 1978. 17(7): p. 1759-1772.

209. Ruzgas, T.A., V.J. Razumas, and J.J. Kulys, Ellipsometric Immunosensors for the Determination of Gamma-Interferon and Human Serum-Albumin. Biosens. Bioelectron., 1992. 7(4): p. 305-308.

210. Caruso, F., E. Rodda, and D.N. Furlong, Orientational aspects of antibody immobilization and immunological activity on quartz crystal microbalance electrodes. J. Colloid Interface Sci., 1996. 178(1): p. 104-115.

211. Shi, L. and K.D. Caldwell, Mucin adsorption to hydrophobic surfaces. J. Colloid Interface Sci., 2000. 224(2): p. 372-381.

212. Santos, J.H., et al., Time-resolved optical waveguide spectroscopy for studying protein adsorption kinetics. Materials Transactions, 2004. 45(4): p. 1015-1018.

213. Rahn, J.R. and R.B. Hallock, Antibody-Binding to Antigen-Coated Substrates Studied with Surface-Plasmon Oscillations. Langmuir, 1995. 11(2): p. 650-654.

214. Hibbert, D.B., J.J. Gooding, and P. Erokhin, Kinetics of irreversible adsorption with diffusion: Application to biomolecule immobilization. Langmuir, 2002. 18(5): p. 1770-1776. 


\section{Vita}

Yan Zhang was born in Beijing, China, in 1973. He received the B.S. in Modern Applied Physics and M.S. in Optics from Tsinghua University of China in 1997 and 2000, respectively. He is expecting his Ph.D. degree in Electrical Engineering from Virginia Polytechnic Institute and State University (Virginia Tech) in December 2005.

He joined the Center for Photonics Technology at Virginia Tech in 2000 as a graduate research assistant. He has authored and co-authored more than 10 papers and patent disclosures. His current research interest are in the area of fiber optic sensors, polyelectrolyte self-assembly, biomedical sensing, and biomedical imaging.

Mr. Zhang is a member of OSA and SPIE. 